\title{
Lectures on Stability and Constant Scalar Curvature*
}

\author{
D.H. Phong and Jacob Sturm
}

\begin{abstract}
An introduction is provided to some current research trends in stability in geometric invariant theory and the problem of Kähler metrics of constant scalar curvature. Besides classical notions such as Chow-Mumford stability, the emphasis is on several new stability conditions, such as K-stability, Donaldson's infinite-dimensional GIT, and conditions on the closure of orbits of almost-complex structures under the diffeomorphism group. Related analytic methods are also discussed, including estimates for energy functionals, Tian-Yau-Zelditch approximations, estimates for moment maps, complex Monge-Ampère equations and pluripotential theory, and the Kähler-Ricci flow.
\end{abstract}

\section{Contents}

1. Introduction 103

2. The conjecture of Yau 105

2.1. Constant scalar curvature metrics in a given Kähler class 106

2.2. The special case of Kähler-Einstein metrics 106

2.3. The conjecture of Yau 107

3. The analytic problem 108

3.1. Fourth order non-linear PDE and Monge-Ampère equations 108

3.2. Geometric heat flows 109

3.3. Variational formulation and energy functionals 109

3.3.1. The Mabuchi $K$-energy $K_{\omega_{0}}(\phi) \quad 109$

3.3.2. The Aubin-Yau functional $F_{\omega_{0}}^{0}(\phi) \quad 110$

4. The spaces $\mathcal{K}_{k}$ of Bergman metrics 111

4.1. Kodaira imbeddings 111

4.2. The Tian-Yau-Zelditch theorem 112

${ }^{*}$ Research supported in part by National Science Foundation grants DMS-02-45371 and DMS-05-14003. Contribution to Current Developments in Mathematics 2007, Harvard University, November 16-17, 2007. 
5. The functional $F_{\omega_{0}}^{0}(\phi)$ on $\mathcal{K}_{k}$

5.1. $F_{\omega_{0}}^{0}$ and balanced imbeddings 114

5.2. $\quad F_{\omega_{0}}^{0}$ and the Euler-Lagrange equation $R-\bar{R}=0 \quad 116$

5.3. $F_{\omega_{0}}^{0}$ and Monge-Ampère masses 116

6. Notions of Stability 117

6.1. Stability in GIT 117

6.1.1. Closedness of orbits 118

6.1.2. The Hilbert-Mumford criterion 119

6.1.3. Chow-Mumford and K-stability 120

6.1.4. Deligne pairings and energy functionals 121

6.1.5. Deligne pairings and Knudsen-Mumford expansions 122

6.1.6. Test configurations and Donaldson-Futaki invariants 123

6.1.7. Equivariant imbeddings of test configurations $\quad 125$

6.1.8. Bott-Chern secondary characteristic classes $\quad 126$

6.2. Donaldson's infinite-dimensional GIT 127

6.2.1. Geodesic segments and geodesic rays 128

6.2.2. Stability conditions in terms of geodesic rays 128

6.3. Stability conditions on $\operatorname{Diff}(X)$ orbits 129

6.3.1. Condition (B) 129

6.3.2. Condition (S) 130

7. The Necessity of Stability 130

7.1. The Moser-Trudinger inequality and analytic $K$-stability 131

7.2. Necessity of Chow-Mumford stability 132

7.2.1. Approximately balanced imbeddings 133

7.2.2. Estimates for the normal projection 134

7.2.3. Synthesis 135

7.3. Necessity of semi $K$-stability 136

8. Sufficient Conditions: the Kähler-Einstein Case 140

8.1. The $\alpha$-invariant 140

8.2. Nadel's multiplier ideal sheaves criterion $\quad 140$

8.3. The Kähler-Ricci flow 142

8.3.1. Perelman's estimates 143

8.3.2. Energy functionals and the Kähler-Ricci flow 144

8.3.3. Perelman's convergence theorem 148

8.3.4. Condition (B) 148

8.3.5. Condition (S) 150

8.3.6. Multiplier ideal sheaves 152

9. General $L$ : Energy functionals and Chow points 153

9.1. $F_{\omega}^{0}$ and Chow points 153

9.2. $K_{\omega}$ and Chow points 155

10. General $L$ : the Calabi energy and the Calabi flow 156

10.1. The Calabi flow 156

10.2. Extremal metrics and stability 157 
11. General $L$ : toric varieties

11.1. Symplectic potentials 160

11.2. $K$-stability on toric varieties $\quad 160$

11.3. The $K$-unstable case 161

12. Geodesics in the space $\mathcal{K}$ of Kähler potentials 161

12.1. The Dirichlet problem for the complex Monge-Ampère equation

12.2. Method of elliptic regularization and a priori estimates 162

12.3. Geodesics in $\mathcal{K}$ and geodesics in $\mathcal{K}_{k} \quad 165$

12.3.1. An Ansatz for geodesic approximations 165

12.3.2. Construction of geodesic segments 167

12.3.3. Construction of geodesic rays 168

12.3.4. Variations on the Ansatz and rates of convergence

\section{Introduction}

A central theme in geometry is to try and characterize a given geometric structure by a metric with "best" curvature properties. A classic example is the uniformization theorem, which says that a complex structure on a compact surface can be characterized by a metric of constant curvature. More recently, in the case of holomorphic vector bundles over a compact Kähler manifold, the algebraic-geometric notion of stability in the sense of Mumford-Takemoto has been shown by Donaldson $[\mathbf{4 3}]$ and Uhlenbeck-Yau [152] to be equivalent to the existence of a Hermitian-Einstein metric.

In general, a metric may not be characterized by curvature properties, since the number of degrees of freedom may not even match. But in Kähler geometry, there is a natural such question: Given a positive line bundle $L \rightarrow X$ over a compact complex manifold $X$, determine when there exists a Kähler form $\omega \in c_{1}(L)$ with constant scalar curvature

$$
R=\mu n \text {. }
$$

Here we have denoted the dimension of $X$ by $n$ and $\mu$ is a constant. When $L=K_{X}^{-\mu}$, it is easy to see that the condition that $R=\mu n$ is equivalent to the Kähler-Einstein condition,

$$
R_{\bar{k} j}=\mu g_{\bar{k} j}
$$

so that the search for metrics of constant scalar curvature also encompasses the famous problem of finding Kähler-Einstein metrics. This problem was solved by Yau [157] and Aubin [5] when $c_{1}(X)<0$. It was solved by Yau [157] when $c_{1}(X)=0$, as part of his solution of the Calabi conjecture. When $c_{1}(X)>0$, the surface case was treated in $[\mathbf{1 4 6}, \mathbf{1 4 1}]$, but the general case of higher dimensions is still open. More generally, the main question in the field 
is the conjecture of Yau [159], still open at this time, which says that the existence of a Kähler form $\omega \in c_{1}(L)$ with constant scalar curvature should be equivalent to the stability of $c_{1}(L)$ in the sense of geometric invariant theory.

The conjecture of Yau can be viewed as a version of the DonaldsonUhlenbeck-Yau theorem for manifolds instead of vector bundles. As such, the corresponding partial differential equations are more non-linear than the equations for Hermitian-Einstein metrics. But there is a significant additional difficulty: it is that, unlike the notion of Mumford-Takemoto stability in the case of vector bundles, it is not yet clear what is the correct notion of stability, and in fact, finding this correct notion has to be viewed as a major component of the problem.

The present lecture notes are based on introductory talks on the subject given at the George Kempf Memorial Lectures at Johns Hopkins University in October 2007, and at the Current Developments in Mathematics conference at Harvard University in November 2007. An earlier and shorter version of the lecture notes had been circulated informally at Harvard at the time of the conference. Their goal is to provide an accessible introduction to the various notions of stability which have been introduced in the context of constant scalar curvature metrics, as well as to some corresponding analytic results. Besides the classical notions of Chow-Mumford and Hilbert-Mumford stability, we discuss the notions of analytic and algebraic $K$-stability due respectively to Tian [143] and Donaldson [47], Donaldson's infinite-dimensional GIT [44], and stability conditions such as (B) and (S) which arise naturally in the context of the Kähler-Ricci flow $[\mathbf{1 1 2}, \mathbf{1 0 5}]$. We also discuss several analytic methods, including inequalities for energy functionals, Tian-Yau-Zelditch approximations, estimates for moment maps, degenerate complex Monge-Ampère equations, and the Kähler-Ricci flow.

Certain aspects of our presentation may be worth mentioning:

(a) It is well-known that the Mabuchi $K$-energy functional plays a central role in the theory, first in the variational formulation of the analytic problem, and second as an important link to the notion of $K$-stability. It does not appear to be as widely appreciated that the Aubin-Yau functional $F_{\omega_{0}}^{0}$ functional plays a similar role, partly because the Euler-Lagrange equation for $F_{\omega_{0}}^{0}$ seems unrelated to the constant scalar curvature equation, and the relevance of $F_{\omega_{0}}^{0}$ can only be seen upon restriction to the spaces $\mathcal{K}_{k}$ of Bergman metrics. We have thus tried to stress these points, by grouping in a single chapter $\S 5$ its key properties: the classic result of Zhang [166] identifying the critical points of $F_{\omega_{0}}^{0}$ on $\mathcal{K}_{k}$ with balanced imbeddings, and thus establishing a bridge to Chow-Mumford stability; the observation due to Donaldson [50] that $R-\mu n$ can indeed be interpreted as the Euler-Lagrange equation for $F_{\omega_{0}}^{0}$ restricted to $\mathcal{K}_{k}$; and the basic relation between $F_{\omega_{0}}^{0}$ and Monge-Ampère masses for a path in the space $\mathcal{K}$ of Kähler potentials [112]. 
(b) In $\S 7.2$, we have simplified to some extent some technical aspects of Donaldson's original proof [45] of the necessity of Chow-Mumford stability. Of course, the underlying motivation from symplectic geometry remains, in particular the key insight of Donaldson, based on Lu's formula [85], that the constancy of the scalar curvature should be closely related to the constancy of the density of states. But the simplification of some important estimates which no longer requires the formalism of infinite-dimensional moment maps may make the proof more accessible.

(c) In $\$ 8.3 .1-8.3 .3$, we have taken the opportunity to discuss certain aspects of the Kähler-Ricci flow which seem not to have been covered fully in the literature. In particular, we describe inequalities between energy functionals along the Kähler-Ricci flow. Combined with Perelman's recent results $[\mathbf{1 0 1}]$ and subsequent uniform Sobolev estimates $[\mathbf{1 6 1}, \mathbf{1 6 5}]$, they readily imply a version of Perelman's unpublished result, namely, that when $\operatorname{Aut}^{0}(X)=0$, the existence of a Kähler-Einstein metric implies the convergence in $C^{\infty}$ of the Kähler-Ricci flow.

(d) In $\S 12.3 .1$, we have extracted from $[\mathbf{1 1 2}, \mathbf{1 1 3}]$ a more general version of the Ansatz producing generalized solutions of the degenerate complex Monge-Ampère equation than was originally stated in these papers. We would like to thank S. Zelditch for stressing to us that such a version may be of interest.

The field is vast and developing very rapidly, and our list of topics is necessarily very incomplete. For example, there is a very rich literature on geometric constructions of metrics of constant scalar curvature, which we did not discuss, see e.g. $[\mathbf{2}, \mathbf{3}, \mathbf{7 6}, \mathbf{8 3}, \mathbf{1 1 7}, \mathbf{1 2 2}]$. There is also a related exposition of O. Biquard in the séminaire N. Bourbaki 2004-2005 [13]. Nevertheless, we hope that these notes can be useful to students interested in getting a quick sense for certain trends in the subject.

\section{The conjecture of Yau}

We begin with some background and notation. Let $X$ be a compact complex manifold of dimension $n$. A Kähler form is a $(1,1)$ form $\omega=$ $\frac{i}{2} g_{\bar{k} j} d z^{j} \wedge d \bar{z}^{k}$ on $X$ which is closed and strictly positive. The Ricci curvature tensor $R_{\bar{k} j}$ and the scalar curvature $R$ of the corresponding metric $g_{\bar{k} j}$ are given by

$$
R_{\bar{k} j}=-\partial_{j} \partial_{\bar{k}} \log \omega^{n}, \quad R=g^{j \bar{k}} R_{\bar{k} j} .
$$

The Ricci curvature form $\operatorname{Ric}(\omega)$ is the $(1,1)$ form defined by

$$
\operatorname{Ric}(\omega)=\frac{i}{2} R_{\bar{k} j} d z^{j} \wedge d \bar{z}^{k}
$$

Since $\omega^{n}$ is a metric on the anti-canonical bundle $K_{X}^{-1}, \operatorname{Ric}(\omega)=-\frac{i}{2} \partial \bar{\partial} \log \omega^{n}$ can be viewed as the curvature form of $K_{X}^{-1}$ with respect to the metric $\omega^{n}$, 
and, as such, must be in the cohomology $\operatorname{class}^{1} c_{1}\left(K_{X}^{-1}\right) \equiv c_{1}(X)$. Sometimes, we also denote the scalar curvature $R$ by $R=R(\omega)$, to emphasize its dependence on the Kähler form $\omega$.

2.1. Constant scalar curvature metrics in a given Kähler class. Let now $L \rightarrow X$ be a positive line bundle, that is, a holomorphic line bundle admitting a metric $h_{0}$ whose curvature $\omega_{0} \equiv-\frac{i}{2} \partial \bar{\partial} \log h_{0} \in c_{1}(L)$ is a positive $(1,1)$-form. Then $\omega_{0}$ equips $X$ with a Kähler structure. The main question addressed in the present lecture series is whether there exists a Kähler form $\omega \in c_{1}(L)$ whose corresponding scalar curvature $R(\omega)$ is constant.

The condition of constant scalar curvature is not particularly rigid for a general Riemannian metric, since it is a single scalar condition on an object with a much higher number of degrees of freedom. However, the situation changes drastically with the above additional constraint that the metric be a Kähler metric in a given Kähler class. For example, if $L=K_{X}^{-1}$, then we have the equivalence

$$
R(\omega)=n \Longleftrightarrow \operatorname{Ric}(\omega)=\omega,
$$

for $\omega \in c_{1}(L)$. Indeed, as we noted above, for any Kähler form $\omega$, we have $\operatorname{Ric}(\omega) \in c_{1}\left(K_{X}^{-1}\right)$. If we require that $\omega \in c_{1}\left(K_{X}^{-1}\right)$, then $\operatorname{Ric}(\omega)$ and $\omega$ are cohomologous, and by the $\partial \bar{\partial}$ lemma, we have

$$
\operatorname{Ric}(\omega)-\omega=\frac{i}{2} \partial \bar{\partial} f
$$

for some $f \in C^{\infty}(X)$ defined uniquely up to an additive constant. The function $f$ is called the Ricci potential, and will play an important role in the sequel. Contracting both sides with $g^{j \bar{k}}$, we obtain $R(\omega)-n=\Delta f$. If $R(\omega)$ is constant, the left hand side of this identity is constant. But the range of $\Delta$ is orthogonal to constants, and thus this constant must be 0 . The function $f$ is then constant, and hence $\operatorname{Ric}(\omega)-\omega=0$. This establishes the claim.

2.2. The special case of Kähler-Einstein metrics. The problem of finding Kähler-Einstein metrics, that is, metrics satisfying

$$
\operatorname{Ric}(\omega)=\mu \omega,
$$

is one of the most celebrated problems in complex geometry. Here $\mu$ is a constant that can be normalized to be $-1,0$, or 1 . When $\mu \neq 0$, the KählerEinstein problem is the special case of the constant scalar curvature problem for metrics in the Kähler class $c_{1}(L)$, with $L=K_{X}^{-\mu}$. The case $\mu=0$ is a special case of the Calabi conjecture, which asserts that for any compact Kähler manifold $(X, \omega)$, and any given $(1,1)$-form $T \in c_{1}(X)$, there exists within the Kähler class of $\omega$ a metric with Ricci curvature equal to $T$.

\footnotetext{
${ }^{1}$ Strictly speaking, $\operatorname{Ric}(\omega) \in \pi c_{1}(X)$. We omit such factors of $\pi$ for notational simplicity.
} 
The Kähler-Einstein problem when $\mu=-1$ was solved independently by Yau [157] and Aubin [5]. The Calabi conjecture was solved by Yau [157]. However, the case $\mu=1$ is still open, and henceforth we refer only to this case when we speak of the Kähler-Einstein problem.

In general, there are obstructions to the existence of Kähler-Einstein metrics of positive scalar curvature. A classic obstruction is the theorem of Matsushima [94], which says that if there exists a constant scalar curvature metric, then the automorphism group of $X$ would have to be reductive. Another obstruction, due to Futaki [57] and also related to the automorphisms of $X$, is the vanishing of the Futaki invariant defined as follows. Given any Kähler metric $\omega \in c_{1}(X)$, let $f$ be its Ricci potential, as defined earlier by (2.4). Then the Futaki invariant Fut is the character on $H^{0}\left(X, T^{1,0}\right)$ defined by

$$
F u t(V)=\int_{X}(V f) \omega^{n}, \quad V \in H^{0}\left(X, T^{1,0}\right) .
$$

The key propery of $F u t(V)$ is that it is actually independent of the choice of $\omega$ within $c_{1}(X)$. Thus, if $c_{1}(X)$ admits a Kähler-Einstein metric, then Fut must vanish identically.

The vanishing of the Futaki invariant was shown to imply the existence of a Kähler-Einstein metric when $\operatorname{dim} X=2[\mathbf{1 4 1}]$ and when $X$ is a toric variety [154]. However, a counterexample was provided by Tian in 1997 [143] of a compact complex manifold $X$ with $c_{1}(X)>0$, no holomorphic vector fields, and yet no Kähler-Einstein metrics (see $\S 7.1$ for a description of this and some other results in $[\mathbf{1 4 3}])$.

2.3. The conjecture of Yau. The existence of Kähler-Einstein metrics, and more generally, of constant scalar curvature metrics in a given Kähler class $c_{1}(L)$, is expected to be related to deeper properties of the bundle $L \rightarrow X$. In fact, the guiding light of much of the current research in the area has been the conjecture of Yau [159], which says that the existence of $\omega \in c_{1}(L)$ with $R(\omega)$ constant should be equivalent to the stability of $L \rightarrow X$ in the sense of geometric invariant theory.

This conjecture has clearly a strong analogy with the case of holomorphic vector bundles. Let $E \rightarrow X$ be a holomorphic vector bundle over a compact Kähler manifold $(X, \omega)$. Given a Hermitian metric $H_{\bar{\alpha} \beta}$ on $E$, let $F_{\bar{k} j}{ }^{\alpha}{ }_{\beta}=-\partial_{\bar{k}}\left(H^{\alpha \bar{\gamma}} \partial_{j} H_{\bar{\gamma} \beta}\right)$ be its curvature. A metric $H_{\bar{\alpha} \beta}$ is said to be Hermitian-Einstein if $g^{j \bar{k}} F_{\bar{k} j}{ }^{\alpha}{ }_{\beta}=\mu \delta^{\alpha}{ }_{\beta}$ for some constant $\mu$. The theorem of Donaldson-Uhlenbeck-Yau $[\mathbf{4 3}, \mathbf{1 5 2}]$ asserts that $E \rightarrow X$ admits a Hermitian-Einstein metric if and only if $E \rightarrow X$ is stable in the sense of Mumford-Takemoto.

As in the case of Hermitian-Einstein metrics and Mumford-Takemoto stability, a particularly striking aspect of the conjecture of Yau is that it asserts the equivalence between the existence of a solution to a non-linear 
partial differential equation and a global, algebraic-geometric property of the underlying space.

\section{The analytic problem}

In this section, we begin by setting up the problem from the analytic point of view. Analytically, there are several possible formulations and approaches.

\subsection{Fourth order non-linear PDE and Monge-Ampère equa-} tions. The most direct formulation of the problem is as a non-linear PDE in the potential $\phi$. More precisely, fixing a metric $h_{0}$ on $L$ with curvature $\omega_{0}=-\frac{i}{2} \partial \bar{\partial} \log h_{0}>0$, we seek another metric $h=h_{0} e^{-\phi}$ with curvature $\omega=-\frac{i}{2} \partial \bar{\partial} \log h>0$ so that $R(\omega)$ is constant. This means that $\phi$ must satisfy the " $\omega_{0}$-plurisubharmonicity" constraint

$$
\omega_{0}+\frac{i}{2} \partial \bar{\partial} \phi>0
$$

and the partial differential equation

$$
-g^{j \bar{k}} \partial_{j} \partial_{\bar{k}} \log \left(\omega_{0}+\frac{i}{2} \partial \bar{\partial} \phi\right)^{n}=\bar{R}
$$

where $g_{\bar{k} j}$ is the metric corresponding to the Kähler form $\omega$. The value of the constant $\bar{R}$ is cohomological: clearly, $\bar{R}$ must be given by the average of the scalar curvature, and thus

$$
\bar{R}=\frac{1}{V} \int_{X} R \omega^{n}=\frac{n}{V} \int_{X} \operatorname{Ric}(\omega) \wedge \omega^{n-1}=n \frac{\left[c_{1}(X)\right]\left[c_{1}(L)\right]^{n-1}}{\left[c_{1}(L)\right]^{n}} \equiv n \mu .
$$

Here $V$ is the volume of $X$ and $\mu$ is the cohomological constant defined by

$$
V=\int_{X} \omega^{n}=\left[\pi c_{1}(L)\right]^{n}, \quad \mu=\frac{\left[c_{1}(X)\right]\left[c_{1}(L)\right]^{n-1}}{\left[c_{1}(L)\right]^{n}} .
$$

Thus the problem can be viewed as a 4th-order non-linear elliptic PDE in $\phi$.

In the special case $L=K_{X}^{-1}$, we have seen that the constant scalar curvature condition is equivalent to the constant Ricci curvature condition. This last condition is well-known to be equivalent to a complex elliptic Monge-Ampère equation. Indeed, we always have $\operatorname{Ric}\left(\omega_{0}\right) \in c_{1}\left(K_{X}^{-1}\right)$, and if $\omega_{0} \in c_{1}(L)=c_{1}\left(K_{X}^{-1}\right)$ also, then we can write $\operatorname{Ric}\left(\omega_{0}\right)-\omega_{0}=\frac{i}{2} \partial \bar{\partial} f_{0}$, where $f_{0}$ is the Ricci potential of $\omega_{0}$. It is now easily seen, simply by taking $-\partial \bar{\partial} \log$ of both sides, that the following complex Monge-Ampère equation

$$
\left(\omega_{0}+\frac{i}{2} \partial \bar{\partial} \phi\right)^{n}=e^{f_{0}-\phi} \omega_{0}^{n}
$$

for $\phi$ still satisfying the $\omega_{0}$-plurisubharmonicity constraint $\omega_{0}+\frac{i}{2} \partial \bar{\partial} \phi>0$, is equivalent to the constant Ricci curvature equation. Geometrically, this means that, when $L=K_{X}^{-1}$, the 4 th order constant scalar curvature equation on the potential can been reduced to a 2 nd order equation in $\phi$, involving the volume of the Kähler metric $\omega=\omega_{0}+\frac{i}{2} \partial \bar{\partial} \phi$. 
3.2. Geometric heat flows. One approach to finding a solution to a given equation is to interpret it as the fixed point of a dynamical system. In the present case, this amounts to replacing the elliptic non-linear PDE by a non-linear parabolic flow. The problem becomes then that of the long-time existence and convergence of the flow.

For general $L$, a parabolic version of the equation (3.2) is the following 4th order parabolic flow,

$$
\dot{\phi}=R-\bar{R}, \quad \phi(0)=c_{0},
$$

for $\phi$ satisfying the $\omega_{0}$-plurisubharmonicity constraint (3.1). This is equivalent to the Calabi flow [20], which is the following flow for metrics $g_{\bar{k} j}$,

$$
\dot{g}_{\bar{k} j}=\partial_{j} \partial_{\bar{k}} R, \quad g_{\bar{k} j}(0)=g_{\bar{k} j}^{0} .
$$

Just as in the elliptic case, when $L=K_{X}^{-1}$, we need only consider a second-order parabolic flow, namely the Kähler-Ricci flow. This is the Kähler version of the Ricci flow introduced by Hamilton [68] (see also $[\mathbf{3 6}, \mathbf{3 7}]$ for a detailed treatment, with an extensive list of references). It can be written either as a flow of metrics,

$$
\dot{g}_{\bar{k} j}=-\left(R_{\bar{k} j}-\mu g_{\bar{k} j}\right), \quad g_{\bar{k} j}(0)=g_{\bar{k} j}^{0}
$$

(with $\mu$ the cohomological value defined by (7.36)), or as a parabolic MongeAmpère equation for the potential $\phi$,

$$
\dot{\phi}=\log \frac{\left(\omega_{0}+\frac{i}{2} \partial \bar{\partial} \phi\right)^{n}}{\omega_{0}^{n}}+\mu \phi-f_{0}, \quad \phi(0)=c_{0},
$$

with $f_{0}$ the Ricci potential (c.f. $\left.(2.4)\right)$ for the original Kähler form $\omega_{0}$.

3.3. Variational formulation and energy functionals. Another approach which plays an important role in the sequel is the variational approach. Thus we seek a functional whose Euler-Lagrange equation is precisely the given equation, so that the problem reduces to determining whether the functional admits a critical point.

3.3.1. The Mabuchi K-energy $K_{\omega_{0}}(\phi)$. A first important fact in the theory, established by Mabuchi [90], is that the equation $R(\omega)-\bar{R}=0$ for $\omega$ in a given Kähler class $c_{1}(L), L \rightarrow X$ positive, is indeed realizable as an EulerLagrange equation. More precisely, there is a functional $K_{\omega_{0}}(\phi)$, now called the Mabuchi $K$-energy and defined on the space $\mathcal{K}$ of Kähler potentials,

$$
\mathcal{K} \equiv\left\{\phi \in C^{\infty}(X) ; \omega_{\phi} \equiv \omega_{0}+\frac{i}{2} \partial \bar{\partial} \phi>0\right\}
$$

satisfying

$$
\delta K_{\omega_{0}}(\phi)=-\frac{1}{V} \int_{X} \delta \phi(R-\bar{R}) \omega_{\phi}^{n}
$$

Since the $K$-energy is characterized by its variation, its exact definition depends on the choice of a reference metric, which we have taken to be $\omega_{0}$. 
An explicit expression for $K_{\omega_{0}}(\phi)$ is (see e.g. the derivation in Theorem 5 below)

$$
K_{\omega_{0}}(\phi)=\frac{1}{V}\left[\int_{X}\left(\log \frac{\omega_{\phi}^{n}}{\omega_{0}^{n}}\right) \omega_{\phi}^{n}-\phi \sum_{j=0}^{n-1} \operatorname{Ric}\left(\omega_{0}\right) \omega_{\phi}^{j} \omega_{0}^{n-1-j}+\mu \sum_{j=0}^{n} \phi \omega_{\phi}^{j} \omega_{0}^{n-j}\right],
$$

although we shall mostly use only its characterizing variational property (3.11). We note that $K_{\omega_{0}}(\phi+c)=K_{\omega_{0}}(\phi)$ for constant $c$, so that $K_{\omega_{0}}(\phi)$ descends to a functional on the space of Kähler metrics in $c_{1}(L)$. Also, the variational formula for $K_{\omega_{0}}(\phi)$ implies the following important cocycle property for $\phi, \phi+\psi \in \mathcal{K}$,

$$
K_{\omega_{0}}(\phi+\psi)=K_{\omega_{0}}(\phi)+K_{\omega_{\phi}}(\psi) .
$$

3.3.2. The Aubin-Yau functional $F_{\omega_{0}}^{0}(\phi)$. There is another functional in the theory, the Aubin-Yau functional $F_{\omega_{0}}^{0}(\phi)$, whose role is as important as that of the Mabuchi $K$-energy, but which is more subtle. First, let $J_{\omega_{0}}(\phi)$ be the functional on $\mathcal{K}$ given by

$$
J_{\omega_{0}}(\phi)=\frac{i}{2 V} \sum_{j=0}^{n-1} \frac{n-j}{n+1} \int_{X} \partial \phi \wedge \bar{\partial} \phi \wedge \omega_{\phi}^{n-1-j} \wedge \omega_{0}^{j} .
$$

Then the functional $F_{\omega_{0}}^{0}(\phi)$ is defined by

$$
F_{\omega_{0}}^{0}(\phi)=J_{\omega_{0}}(\phi)-\frac{1}{V} \int_{X} \phi \omega_{0}^{n} .
$$

Its variation can be readily verified to be

$$
\delta F_{\omega_{0}}^{0}(\phi)=-\frac{1}{V} \int_{X} \delta \phi \omega_{\phi}^{n}
$$

and it also satisfies the cocycle condition

$$
F_{\omega_{0}}^{0}(\phi+\psi)=F_{\omega_{0}}^{0}(\phi)+F_{\omega_{\phi}}^{0}(\psi), \quad \phi, \phi+\psi \in \mathcal{K} .
$$

In the case of $L=K_{X}^{-1}$, the Monge-Ampère equation (3.5) for KählerEinstein metrics can be viewed as an Euler-Lagrange equation for $F_{\omega_{0}}^{0}(\phi)$ with constraints. More precisely, let

$$
F_{\omega_{0}}(\phi)=F_{\omega_{0}}^{0}(\phi)-\log \left(\frac{1}{V} \int_{X} e^{f_{0}-\phi} \omega_{0}^{n}\right)
$$

Then we have

$$
\frac{\delta F_{\omega_{0}}}{\delta \phi}=0 \Longleftrightarrow\left(\omega_{0}+\frac{i}{2} \partial \bar{\partial} \phi\right)^{n}=e^{f_{0}-\phi} \omega_{0}^{n} .
$$

For general $L$, there does not appear to be any evident relation between $F_{\omega_{0}}^{0}(\phi)$ and the constant scalar curvature equation $R(\omega)-\bar{R}=0$. It comes therefore as a surprise that $F_{\omega_{0}}^{0}(\phi)$ is actually intimately related to this equation. However, to see this relation, we have to restrict $F_{\omega_{0}}^{0}(\phi)$ to certain 
finite-dimensional subspaces of $\mathcal{K}$, namely the subspaces $\mathcal{K}_{k}$ of Bergman metrics. We turn next to a description of these spaces.

\section{The spaces $\mathcal{K}_{k}$ of Bergman metrics}

The conjecture of Yau links the existence of a transcendental object, namely a Kähler metric with constant scalar curvature, to an algebraicgeometric condition, namely stability in GIT. This last concept depends fundamentally on the realization of $X$ as a projective variety by Kodaira imbeddings. Associated to each such imbedding is an "algebraic" metric on $L$, namely the induced Fubini-Study metric from $O(1)$. The Tian-YauZelditch theorem (TYZ) asserts that any metric on $L$ with positive curvature can be approximated by such "algebraic" metrics. Thus the induced Fubini-Study metrics - or "Bergman metrics" - provide a bridge between the original analytic problem and the underlying algebraic-geometric structure. The strategy of approximating transcendental objects by algebraicgeometric ones has been advocated by Yau [159] over the years. It plays an essential role in many developments described in this paper, in particular Donaldson's theorem on the necessity of Chow-Mumford stability in $\S 7.2$, the construction of geodesics in section $\S 12.3$, and Donaldson's lower bound for the Calabi functional in $§ 7.3$. In this section, we describe the spaces of Bergman metrics and the TYZ theorem. In the next, we return to the functional $F_{\omega_{0}}^{0}(\phi)$ and show how its significance in connection with the equation $R-\bar{R}=0$ emerges upon restricting it to the spaces of Bergman metrics.

4.1. Kodaira imbeddings. Let $L \rightarrow X$ be a positive line bundle over a compact complex manifold $X$. Then for each basis $\underline{s}=\left\{s_{\alpha}(z)\right\}_{\alpha=0}^{N_{k}}$ of $H^{0}\left(X, L^{k}\right), N_{k}+1=\operatorname{dim} H^{0}\left(X, L^{k}\right)$, and $k$ large enough, the Kodaira imbedding theorem asserts that the map

$$
\iota_{\underline{s}}: X \in z \longrightarrow\left[s_{0}(z), \ldots, s_{N_{k}}(z)\right] \in \mathbf{C P}^{N_{k}}
$$

is an imbedding. Under this imbedding, the bundle $O(1)$ over $\mathbf{C P}^{N_{k}}$ pullsback to $L^{k}$. Let $h_{F S}$ be the Fubini-Study metric on $O(1)$, and $\omega_{F S}=$ $-\frac{i}{2} \partial \bar{\partial} \log h_{F S}$ the Fubini-Study metric on $\mathbf{C P}^{N_{k}}$. Then $\iota_{\underline{s}}^{*}\left(h_{F S}\right)^{1 / k}$ is a metric of $L$ with positive curvature, and the space $\mathcal{K}_{k}$ of Bergman metrics is by definition the space of all metrics of the form $\iota_{\underline{s}}^{*}\left(h_{F S}\right)^{1 / k}$ as the basis $\underline{s}$ varies,

$$
\mathcal{K}_{k}=\left\{\iota_{\underline{s}}^{*}\left(h_{F S}\right)^{1 / k} ; \underline{s} \text { basis of } H^{0}\left(X, L^{k}\right)\right\}
$$

If we fix a reference basis $\underline{\hat{s}}$, then any other basis can be obtained from $\underline{\hat{s}}$ by an element of $G L\left(N_{k}+1\right)$, and, since $h_{F S}$ is invariant under $S U\left(N_{k}+1\right)$, we obtain

$$
\mathcal{K}_{k}=S L\left(N_{k}+1\right) / S U\left(N_{k}+1\right) .
$$

Thus $\mathcal{K}_{k}$ can be viewed as a symmetric space with negative curvature. 
4.2. The Tian-Yau-Zelditch theorem. Explicitly, we can view $\mathbf{C P}^{N}$ as $\mathbf{C P}^{N}=\left\{\mathbf{C}^{N+1} \backslash 0\right\} / \sim$ with $x, y \in \mathbf{C}^{N+1} \backslash 0$ equivalent if $x=\lambda y$. As such, $\mathbf{C P}^{N}$ carries a natural line bundle, namely the universal bundle consisting of the line $\ell_{[y]}=\{x=\lambda y\}$ over the point $[y] \in \mathbf{C P}^{N}$. The $O(1)$ bundle over $\mathbf{C P}^{N}$ is the dual of the universal bundle. Thus its fiber at each $[y]$ is the space of linear functionals $\ell_{[y]} \rightarrow$ C. A basis of $H^{0}\left(\mathbf{C P}^{N}, O(1)\right)$ is provided by the linear functionals $x \rightarrow x_{\alpha}$, for $0 \leq \alpha \leq N$. We can then define the Fubini-Study metrics on $O(1)$ by

$$
h_{F S}=\frac{1}{\sum_{\alpha=0}^{N}\left|x_{\alpha}\right|^{2}} \equiv \frac{1}{|x|^{2}} .
$$

Its curvature $\omega_{F S}=\frac{i}{2} \partial \bar{\partial} \log |x|^{2}$ is then the Fubini-Study metric on $\mathbf{C P}^{N}$.

In the setting $L \rightarrow X$ positive, imbedded into $O(1) \rightarrow \mathbf{C P}^{N_{k}}$ by a basis $\underline{s}$ of $H^{0}\left(X, L^{k}\right)$, it follows that the induced metrics on $L$ and on $X$ are given explicitly by

$$
\iota_{\underline{s}}^{*}\left(h_{F S}\right)^{1 / k}=\frac{1}{\left(\sum_{\alpha=0}^{N_{k}}\left|s_{\alpha}(z)\right|^{2}\right)^{1 / k}}, \quad \frac{1}{k} \iota_{\underline{s}}^{*}\left(\omega_{F S}\right)=\frac{i}{2 k} \partial \bar{\partial} \log \sum_{\alpha=0}^{N_{k}}\left|s_{\alpha}(z)\right|^{2} .
$$

Fix now a metric $h$ on $L$ with positive curvature $\omega=-\frac{i}{2} \partial \bar{\partial} \log h$. Let $\underline{s}$ be an orthonormal basis for $H^{0}\left(X, L^{k}\right)$, with respect to the $L^{2}$ metric defined on the sections of $L^{k}$ by the metric $h$ and the volume form $\omega$. Define the density of states $\rho_{k}(z)$ by

$$
\rho_{k}(z)=\sum_{\alpha=0}^{N_{k}}\left|s_{\alpha}(z)\right|^{2} h^{k}(z)
$$

Clearly, $\rho_{k}(z)$ is independent of the choice of $\underline{s}$ among the orthonormal bases of $H^{0}\left(X, L^{k}\right)$. Its integral gives $N_{k}+1=\operatorname{dim} H^{0}\left(X, L^{k}\right)$, whence its name. Introducing $\rho_{k}(z)$ converts the expression (4.5) for the induced metrics

$$
h(k) \equiv \iota_{\underline{s}}^{*}\left(h_{F S}\right)^{1 / k}, \quad \omega(k) \equiv \frac{1}{k} \iota_{\underline{s}}^{*}\left(\omega_{F S}\right)
$$

into the following simple relation with the original metric $h$ and its curvature $\omega$,

(4.8) $\log \frac{h(k)}{h}=-\frac{1}{k} \log \rho_{k}(z), \quad \omega-\omega(k)=-\frac{i}{2 k} \partial \bar{\partial} \log \rho_{k}(z)$.

The Tian-Yau-Zelditch theorem can be stated as follows:

TheOREM 1. $[\mathbf{1 5 8}, \mathbf{1 4 0}, \mathbf{1 6 3}]$ The density of states $\rho_{k}(z)$ admits an asymptotic expansion $\sum_{p=0}^{\infty} A_{p}(z) k^{n-p}$ with $A_{0}(z)=1, A_{p}(z)$ smooth functions, in the sense that

$$
\left\|\rho_{k}(z)-\sum_{p=0}^{M} A_{p}(z) k^{n-p}\right\|_{C^{L}} \leq C_{L, M} k^{n-M-1} .
$$


In particular, we have the following approximations, for any $C^{L}$ norm $\|\cdot\|$,

$$
\left\|\log \frac{h(k)}{h}+n \frac{\log k}{k}\right\|=O\left(\frac{1}{k^{2}}\right), \quad\|\omega(k)-\omega\|=O\left(\frac{1}{k^{2}}\right) .
$$

The proof of (4.9) is built on the expansion of Boutet de MonvelSjöstrand [17] for the Szegö kernel on strongly pseudoconvex domains, extending an earlier expansion along the diagonal due to Fefferman [55]. Another independent proof, also built on the result of Boutet de MonvelSjöstrand, is due to Catlin [25]. The approach in [140] is based on Hörmander's $L^{2}$ estimates, and a generalization to open manifolds with bounds on the Ricci curvature is also given there. The TYZ theorem implies that an arbitrary metric $h$ in the space $\mathcal{K}$ of Kähler potentials can be approximated in $C^{\infty}$ by metrics $h(k)$ in $\mathcal{K}_{k}$. In this sense, we have

$$
\mathcal{K}=\lim _{k \rightarrow \infty} \mathcal{K}_{k}
$$

The second leading coefficient $A_{1}(z)$ plays a key role in subsequent developments (c.f. $§ 5.2, \oint 7.2$ and $\S 7.3$ ) and has been determined by Lu [86]:

THEOREM 2. Let the set up be the same as in the previous theorem. Then

$$
A_{1}(z)=\frac{1}{2 \pi} R(z)
$$

where $R(z)$ is the scalar curvature of the Kähler metric $\omega$ on $X$.

\section{The functional $\boldsymbol{F}_{\omega_{0}}^{0}(\phi)$ on $\mathcal{K}_{k}$}

We return now to the discussion of the functional $F_{\omega_{0}}^{0}(\phi)$. Unlike the $K$-energy $K_{\omega_{0}}(\phi)$, for general positive line bundles $L \rightarrow X$, there is no obvious relation between $F_{\omega_{0}}^{0}(\phi)$ and the constant scalar curvature equation $R-\bar{R}=0$. However, remarkably, upon restriction to the spaces $\mathcal{K}_{k}$ of Bergman metrics, $F_{\omega_{0}}^{0}(\phi)$ turns out to be closely related both to the constant scalar curvature equation and to stability conditions. In fact, it is related to all forms of GIT stability conditions, whether it be Chow-Mumford, $K$ stability, or Donaldson's infinite-dimensional GIT. We explain this now in some detail.

It is convenient to think of the restriction of $F_{\omega_{0}}^{0}(\phi)$ to $\mathcal{K}_{k}$ as a functional on $S L\left(N_{k}+1\right) / U\left(N_{k}+1\right)$. To this end, we fix a basis $\underline{\hat{s}}$ of $H^{0}\left(X, L^{k}\right)$, and identify $X$ with the projective variety $\iota_{\underline{\hat{s}}}(X)$,

$$
X \longleftrightarrow \hat{X} \equiv \iota_{\underline{\hat{s}}}(X) \subset \mathbf{C P}^{N_{k}}
$$

Now for any basis $\underline{s}=\sigma \cdot \underline{\hat{s}}$ of $H^{0}\left(X, L^{k}\right)$ with $\sigma \in S L\left(N_{k}+1\right)$, we have

$$
\iota_{\sigma \cdot \underline{\hat{s}}}(X)=\sigma \cdot \iota_{\underline{\hat{s}}}(X)
$$

and the pull-back metrics $\iota_{\sigma \cdot \underline{\underline{\hat{s}}}}^{*}\left(\omega_{F S}\right)$ and $\iota_{\underline{\underline{\hat{s}}}}^{*}\left(\omega_{F S}\right)$ are related by

$$
\iota_{\sigma \cdot \underline{\hat{s}}}^{*}\left(\omega_{F S}\right)=\frac{i}{2} \partial \bar{\partial} \log |\sigma x|^{2}=\iota_{\underline{\hat{s}}}^{*}\left(\omega_{F S}\right)+\frac{i}{2} \partial \bar{\partial} \phi_{\sigma}
$$


where $[x] \in \hat{X}$, and $\phi_{\sigma}(x)$ is the following potential

$$
\phi_{\sigma}(x)=\log \frac{|\sigma x|^{2}}{|x|^{2}}, \quad x \in \mathbf{C}^{N_{k}+1} .
$$

Now $\iota_{\underline{s}}^{*}\left(\omega_{F S}\right) \in k c_{1}(L)$, and it is natural to introduce the functional $F_{\iota_{\underline{\underline{s}}}^{*}\left(\omega_{F S}\right)}^{0}$ $(\phi)$, which is a functional on the space of Kähler potentials in $k c_{1}(L)$ rather than in $c_{1}(L)$. The use of $F_{\iota_{\underline{\underline{s}}}^{*}\left(\omega_{F S}\right)}(\phi)$ is particularly convenient when $X$ is identified with $\hat{X}$ and the extrinsic geometry of $\hat{X}$ as a projective variety play an important role. Clearly, we have in general

$$
F_{k \omega_{0}}^{0}(k \phi)=k F_{\omega_{0}}^{0}(\phi), \quad \phi \in \mathcal{K},
$$

so that this scaling from $c_{1}(L)$ to $k c_{1}(L)$ is a question of emphasis rather than one really of substance. Furthermore, in view of the cocycle property (3.17), any other choice of reference Kähler form $\omega_{0}$ in $F_{k \omega_{0}}(k \phi)$ can be brought back to $\iota_{\underline{\hat{s}}}^{*}\left(\omega_{F S}\right)$ when considering the derivatives of $F_{k \omega_{0}}^{0}(k \phi)$. Thus we have the following function of $\sigma$,

$$
S L\left(N_{k}+1\right) \ni \sigma \longrightarrow F_{l_{\underline{s}}^{*}\left(\omega_{F S}\right)}^{0}\left(\phi_{\sigma}\right)
$$

which can be viewed as a rescaling of the functional $F_{\omega_{0}}^{0}(\phi)$ restricted to $\mathcal{K}_{k}$.

5.1. $\boldsymbol{F}_{\boldsymbol{\omega}_{0}}^{\mathbf{0}}$ and balanced imbeddings. A key feature of $\mathcal{K}_{k}=S L\left(N_{k}+\right.$ $1) / S U\left(N_{k}+1\right)$ is that any two potentials there can be connected by a one-parameter subgroup $\sigma(t)=e^{(\delta \sigma) t}$ in $S L\left(N_{k}+1\right), \delta \sigma \in \operatorname{sl}\left(N_{k}+1\right)$. The derivatives of $F_{\iota_{\underline{s}}^{*}\left(\omega_{F S}\right)}^{0}\left(\phi_{\sigma}\right)$ along such one-parameter subgroups can be evaluated explicitly:

$$
\begin{aligned}
-\left.\frac{d}{d t}\right|_{t=0} F_{\iota_{\underline{\underline{s}}}^{*}\left(\omega_{F S}\right)}^{0}\left(\phi_{\sigma(t)}\right) & =\operatorname{Tr}\left(\left(\delta \sigma+\delta \sigma^{*}\right) \cdot M\right), \\
-\left.\frac{d^{2}}{d t^{2}}\right|_{t=0} F_{\iota_{\underline{\underline{s}}}^{*}\left(\omega_{F S}\right)}^{0}\left(\phi_{\sigma(t)}\right) & =\left\|\pi_{\mathcal{N}} V\right\|^{2}
\end{aligned}
$$

where $M=M_{\bar{\alpha} \beta}$ is the following matrix

$$
M_{\bar{\alpha} \beta}=\frac{1}{V_{F S}} \int_{\hat{X}} \frac{\bar{x}_{\alpha} x_{\beta}}{|x|^{2}} \omega_{F S}^{n}
$$

$V_{F S}$ is the volume of $\hat{X}$ with respect to the Fubini-Study metric, and $\pi_{\mathcal{N}} V$ is the projection on the normal bundle to $\hat{X}$ of the vector field corresponding to the action of $\delta \sigma$ on $\mathbf{C P}^{N_{k}}$. In fact, the variation of $\phi_{\sigma}$ with respect to $\sigma$ is given by

$$
\delta \phi_{\sigma}=\frac{x^{*} \sigma^{*}\left(\delta \sigma+\delta \sigma^{*}\right) \sigma x}{x^{*} \sigma^{*} \sigma x} .
$$


and thus, in view of the defining equation (3.16) for $\delta F_{\omega}^{0}(\phi)$, we obtain

$$
\begin{aligned}
\left.\frac{d}{d t}\right|_{t=0} F_{\iota_{\underline{\underline{s}}}^{*}\left(\omega_{F S}\right)}^{0}\left(\phi_{\sigma}\right) & =-\left.\frac{1}{V_{F S}} \int_{X} \dot{\phi}_{\sigma(t)} \iota_{\sigma(t) \cdot \underline{s}}^{*}\left(\omega_{F S}\right)^{n}\right|_{t=0} \\
& =-\frac{1}{V_{F S}} \int_{\hat{X}} \frac{x^{*}\left(\delta \sigma+\delta \sigma^{*}\right) x}{x^{*} x} \omega_{F S}^{n}
\end{aligned}
$$

and the desired formula. The proof of the formula for the second derivative is slightly longer, and can be found in [107]. Note that it implies that $F_{\iota_{\underline{\underline{s}}}^{*}\left(\omega_{F S}\right)}^{0}\left(\phi_{\sigma}\right)$ is convex along one-parameter subgroups of $S L\left(N_{k}+1\right)$. Replacing $\hat{X}$ by $\sigma \cdot \hat{X}$, we obtain the following immediate consequence of the formula for the first derivative:

TheOREM 3. A point $\sigma \in S L\left(N_{k}+1\right)$ is a critical point of the functional $F_{\omega_{0}}^{0}$ restricted to $\mathcal{K}_{k}$ if and only if the image $\sigma \cdot \hat{X}$ of $X$ by the Kodaira imbedding defined by the basis $\sigma \cdot \underline{\hat{s}}$ of $H^{0}\left(X, L^{k}\right)$ is a balanced submanifold of $\mathbf{C P}^{N_{k}}$, in the sense that

$$
\frac{1}{V_{F S}} \int_{\sigma \cdot \hat{X}} \frac{\bar{x}_{\alpha} x_{\beta}}{|x|^{2}} \omega_{F S}^{n}=\lambda \delta_{\bar{\alpha} \beta}
$$

for some scalar $\lambda$.

The notion of a balanced submanifold was introduced by Bourguignon, $\mathrm{Li}$, and Yau [16]. As we shall see later, by a theorem of Zhang [166] (see also Luo [89] and [107]), the existence of a unique balanced imbedding is equivalent to Chow-Mumford stability, so that the above theorem actually provides a first link between the functional $F_{\omega}^{0}(\phi)$ and stability in GIT. A similar version of balanced imbeddings for vector bundles and its equivalence with Gieseker stability has been proved by X. Wang [153].

The convexity of $F_{\iota_{s}^{*}\left(\omega_{F S}\right)}^{0}\left(\phi_{\sigma}\right)$ along one-parameter subgroups provides yet another link to stability, this time to the notion of $K$-stability to be described in greater detail in section $§ 6.1$. Indeed, it implies that for any $\delta \sigma_{k} \in \operatorname{sl}\left(N_{k}+1\right)$, the limit

$$
\mu_{k} \equiv-\lim _{t \rightarrow-\infty} V_{F S} \dot{F}_{\iota_{\underline{s}}^{*}\left(\omega_{F S}\right)}^{0}\left(\phi_{\sigma_{k}(t)}\right)
$$

exists. For the natural family of $\delta \sigma_{k}$ associated to a test configuration, this limit is in fact the Mumford numerical invariant, and we shall see subsequently that

$$
F=\lim _{k \rightarrow \infty} \frac{\mu_{k}}{k^{n}}
$$

is the Donaldson-Futaki invariant, whose sign defines the notion of $K$ stability (see Lemma 6 below). 
5.2. $\boldsymbol{F}_{\boldsymbol{\omega}_{0}}^{\mathbf{0}}$ and the Euler-Lagrange equation $R-\bar{R}=0$. We show next that the equation $R-\bar{R}=0$ is, in a sense, the Euler-Lagrange equation for $F_{\omega}^{0}$ when restricted to $\mathcal{K}_{k}$. For this, fix a metric $h$ on $L$ with positive curvature $\omega$, and let $\underline{\hat{s}}$ be an orthonormal basis for $H^{0}\left(X, L^{k}\right)$ with respect to the $L^{2}$ metric defined by $h$ and the volume form $\omega^{n}$. By definition of the Kodaira imbedding, $x_{\alpha}=s_{\alpha}(z), V_{F S}=k^{n} V$, and the matrix $M_{\bar{\alpha} \beta}$ of (5.8) can be expressed as

$$
M_{\bar{\alpha} \beta}=\frac{1}{V_{F S}} \int_{X} \frac{\bar{s}_{\alpha} s_{\beta}}{\sum_{\gamma=0}^{N_{k}}\left|s_{\gamma}\right|^{2}}\left(\iota_{\underline{\hat{s}}}^{*}\left(\omega_{F S}\right)\right)^{n}=\frac{1}{V} \int_{X} \frac{\left\langle s_{\beta}, s_{\alpha}\right\rangle}{\rho_{k}(z)} \omega^{n} \frac{\left(\frac{1}{k} \iota_{\underline{\underline{s}}}^{*}\left(\omega_{F S}\right)\right)^{n}}{\omega^{n}}
$$

where $\rho_{k}(z)$ is the density of states defined in (4.6). In view of the Tian-YauZelditch theorem and Lu's formula described in section $\S 4$, we can expand the right hand side in powers of $k$ and obtain

$$
M_{\bar{\alpha} \beta}=\kappa \delta_{\bar{\alpha} \beta}-\frac{1}{V k^{n+1}} \int_{X}\left\langle s_{\beta}, s_{\alpha}\right\rangle(R-\bar{R}) \omega^{n}+O\left(\frac{1}{k^{n+2}}\right) .
$$

for some constant $\kappa$ whose exact value is immaterial. Thus we have, under a variation $\delta \sigma \in \operatorname{sl}\left(N_{k}+1\right)$,

$$
\begin{aligned}
\delta F_{\iota_{\underline{\underline{s}}}^{*}\left(\omega_{F S}\right)}^{0}\left(\phi_{\sigma}\right) & =\frac{1}{V k^{n+1}} \int_{X} \delta \Phi(R-\bar{R}) \omega^{n}+O\left(\frac{1}{k^{n+2}}\right) \\
\delta \Phi & \equiv \operatorname{Tr}\left(\left(\delta \sigma+\delta \sigma^{*}\right) \cdot\left\langle s_{\beta}, s_{\alpha}\right\rangle\right)
\end{aligned}
$$

This observation is due to Donaldson [50]. It is in this sense that $R-\bar{R}=0$ can also be viewed as the Euler-Lagrange equation for $F_{\omega_{0}}^{0}$.

5.3. $\boldsymbol{F}_{\boldsymbol{\omega}_{0}}^{\mathbf{0}}$ and Monge-Ampère masses. Another key property of $F_{\omega_{0}}^{0}$ is its relation to Monge-Ampère masses, and hence to infinite-dimensional GIT (see $\S 12$ below). Strictly speaking, this is a property of $F_{\omega_{0}}^{0}$ itself, not necessarily restricted to $\mathcal{K}_{k}$, but we include it in this section, because the main application of this relation does involve restrictions to $\mathcal{K}_{k}$, and also because it fits in the theme of relations to stability which are not apparent from the initial definition of $F_{\omega_{0}}^{0}$.

Let $(-T, 0] \ni t \rightarrow \phi(z, t)$ be a smooth path in the space $\mathcal{K}$ of Kähler potentials. To such a path, we associate the following potential $\Phi(z, w)$ defined on $X \times A$, with $A=\left\{w \in \mathbf{C} ; e^{-T}<|w| \leq 1\right\}$,

$$
\Phi(z, w)=\phi(z, \log |w|)
$$

Let $\Omega_{0}$ be the curvature $\omega_{0}$ of $h_{0}$, viewed as a $(1,1)$-form on $X \times A$. Then we have the following basic identity $[\mathbf{1 1 3}]$

$$
\frac{1}{(n+1) V} \iint_{X \times A}\left(\Omega_{0}+\frac{i}{2} \partial \bar{\partial} \Phi\right)^{n+1}=-\dot{F}_{\omega_{0}}^{0}(\phi(\cdot, 0))+\dot{F}_{\omega_{0}}^{0}(\phi(\cdot, T)) .
$$


In particular, for infinitely extended paths, we may take $T \rightarrow \infty$ and obtain, with $D^{\times}=\{w \in \mathbf{C} ; 0<|w| \leq 1\}$,

$$
\frac{1}{(n+1) V} \iint_{X \times D^{\times}}\left(\Omega_{0}+\frac{i}{2} \partial \bar{\partial} \Phi\right)^{n+1}=-\dot{F}_{\omega}^{0}(\phi(\cdot, 0))+\lim _{T \rightarrow \infty} \dot{F}_{\omega}^{0}(\phi(\cdot, T)) .
$$

The identity (5.18) can be established as follows. A first important observation due to Semmes $[\mathbf{1 2 0}]$ and Donaldson $[\mathbf{4 4}]$ is that

$$
\frac{1}{n+1}\left(\Omega_{0}+\frac{i}{2} \partial \bar{\partial} \Phi\right)^{n+1}=\left(\ddot{\phi}-g_{\phi}^{j \bar{k}} \partial_{j} \dot{\phi} \partial_{\bar{k}} \dot{\phi}\right) \omega_{\phi}^{n} d t d \theta
$$

where $\left(e^{t}, \theta\right)$ are polar coordinates for $w$. On the other hand,

$$
\begin{aligned}
-\partial_{t} F_{\omega}^{0}(\phi(\cdot, t)) & =\partial_{t}\left(\frac{1}{V} \int_{X} \dot{\phi} \omega_{\phi}^{n}\right) \\
& =\frac{1}{V} \int_{X}(\ddot{\phi}+\dot{\phi} \Delta \dot{\phi}) \omega_{\phi}^{n} \\
& =\frac{1}{V} \int_{X}\left(\ddot{\phi}-g_{\phi}^{j \bar{k}} \partial_{j} \dot{\phi} \partial_{\bar{k}} \dot{\phi}\right) \omega_{\phi}^{n} .
\end{aligned}
$$

Comparing the two identities and integrating in $t$ gives the desired formula.

The significance of the identity (5.18) is as follows. We shall see later that the space $\mathcal{K}$ of Kähler potentials carries a natural metric, with respect to which the geodesic equation for paths $t \rightarrow \phi(z, t)$ is precisely the vanishing of the expression $\ddot{\phi}-g_{\phi}^{j \bar{k}} \partial_{j} \dot{\phi} \partial_{\bar{k}} \dot{\phi}$, and hence of $\left(\Omega_{0}+\frac{i}{2} \partial \bar{\partial} \Phi\right)^{n+1}$ in view of the equation (5.20). Just as $\mathcal{K}$ is the limit of the finite-dimensional symmetric spaces $\mathcal{K}_{k}$, the geodesic rays in $\mathcal{K}$ are the analogues in infinite-dimensional GIT of the one-parameter subgroups in $\mathcal{K}_{k}$, and thus the identity (5.18) links this key concept for stability once again with the $F_{\omega_{0}}^{0}$ functional.

\section{Notions of Stability}

Stability is a condition on a geometric object that should ensure that the moduli space of stable objects be a well-behaved space, and in particular Hausdorff. As stressed earlier, it is still unclear what is the correct notion of stability for a positive line bundle $L \rightarrow X$ that should apply in Yau's conjecture, and be equivalent to the existence of constant scalar curvature metric in the Kähler class $c_{1}(L)$. A prime candidate is $K$-stability (or its variants), but other seemingly natural notions of stability also arise, as suggested by infinite-dimensional GIT and the Kähler-Ricci flow. We present here a brief overview of all these notions.

6.1. Stability in GIT. In GIT, one typically associates to the geometric object a non-zero "defining vector" $V$ in a vector space $H$ carrying an action of $S L(N+1, \mathbf{C})$. The vector $V$ is not intrinsically defined, as it depends on various choices, but if $[V] \in \mathbf{P} H$ denotes the image of $V$ 
in $\mathbf{P} H=(H \backslash\{0\}) / \mathbf{C}^{\times}$, then the orbit $S L(N+1) \cdot[V] \subseteq \mathbf{P} H$ is independent of all choices and it characterizes the object. We say that the orbit $S L(N+1) \cdot[V] \subseteq \mathbf{P} H$ is GIT stable if $S L(N+1) \cdot V \subseteq H$ is closed and the stabilizer of $V$ is finite. Thus the orbit of $[V]$ characterizes the object in question while the orbit of $V$ is the one which is relevant in the definition of GIT stability. It follows then that the moduli space of stable orbits is Hausdorff and, in fact, an algebraic variety [97]. For an extensive discussion, see $[\mathbf{1 4 9}]$.

6.1.1. Closedness of orbits. In our specific context, the geometric object is a positive line bundle $L \rightarrow X$ over a compact complex manifold. The GIT procedure outlined above can be implemented as follows:

As before, for $k$ sufficiently large, to each basis $\underline{s}=\left\{s_{\alpha}(z)\right\}_{\alpha=0}^{N_{k}}$ of $H^{0}\left(X, L^{k}\right)$ we can associate the image of $X$ by the corresponding Kodaira imbedding $\iota_{\underline{s}}(X)$, which is a subvariety of $\mathbf{C P}^{N_{k}}$. Clearly, the subvariety $\iota_{s}(X)$ is not intrinsic, since it depends on the choice of the basis $\underline{s}$. If $\underline{\tilde{s}}$ is another basis, then $\underline{\tilde{s}}=\sigma \cdot \underline{s}$ and $\iota_{\underline{s}}(X)=\sigma \cdot \iota_{\underline{s}}(X)$ for some $\sigma \in G L\left(N_{k}+1\right)$. As discussed above, in order to define an intrinsic object in GIT we associate to $X$ the whole orbit

$$
X \longleftrightarrow\left\{\sigma \cdot \iota_{\underline{s}}(X) \mid \sigma \in S L\left(N_{k}+1\right)\right\}
$$

As described above, we would like rather an orbit of vectors $V$ rather than an orbit of projective varieties. In Chow-Mumford stability, this is achieved by associating a Chow point Chow $(\hat{X})$ to each n-dimensional subvariety $\hat{X} \subset \mathbf{C P}^{N}$ of degree $d$ as follows: let the Chow variety $Z$ of $\hat{X}$ be the hypersurface in the Grassmannian $\operatorname{Gr}\left(N-n-1, \mathbf{C P}^{N}\right)$ defined by

$$
Z=\left\{w \in G r\left(N-n-1, \mathbf{C P}^{N}\right) ; w \cap \hat{X} \neq 0\right\}
$$

Let $H$ be the vector space $H=H^{0}(G r(N-n-1), O(d))$ and let $\left[V_{\hat{X}}\right]$ be the set of elements of $H$ which vanish on $Z$. Then $\left[V_{\hat{X}}\right]$ is a line in $H$ (known as the "Chow line") that is, $\left[V_{\hat{X}}\right] \in \mathbf{P} H$. We say that $\hat{X}$ is Chow-Mumford stable if the orbit $S L(N+1) \cdot\left[V_{\hat{X}}\right] \subseteq \mathbf{P} H$ is GIT stable. We say that a polarized variety $(X, L)$ is Chow-Mumford r-stable if $L^{r}$ is very ample and if, for some (and hence every) basis $\underline{s}$ of $H^{0}\left(X, L^{r}\right)$, the subvariety $\iota_{\underline{s}}(X) \subseteq \mathbf{P}^{N_{r}}$ is Chow-Mumford stable in the sense defined above.

To define Hilbert-Mumford stability, one associates a different line to each subvariety $\hat{X}$ of $\mathbf{C P}^{N}$ as follows. For $k>>0$, the inclusion

$$
\left\{s \in H^{0}\left(\mathbf{C P}^{N}, O(k)\right):\left.s\right|_{\hat{X}}=0\right\} \subset H^{0}\left(\mathbf{C P}^{N}, O(k)\right)
$$

defines an element of the Grassmannian of codimension $p(k)$ planes in $H^{0}\left(\mathbf{C P}^{N}, O(k)\right)$, where

$$
p(k) \equiv \operatorname{dim} H^{0}\left(\hat{X},\left.O(k)\right|_{\hat{X}}\right)
$$

is the Hilbert polynomial. The maximum wedge product of this subspace is by definition the Hilbert-Mumford k-line. We say $\hat{X}$ is Hilbert-Mumford $\mathrm{k}$-stable if the orbit of this line is GIT stable. We say $\hat{X}$ is Hilbert-Mumford 
stable if it is k-stable for $k$ sufficiently large. We say that $(L, X)$ is asymptotically Hilbert-Mumford stable if for every $r>>0$, there is a basis $\underline{s}$ of $H^{0}\left(X, L^{r}\right)$ such that $\iota_{\underline{s}}(X) \subseteq \mathbf{C P}^{N_{r}}$ is Hilbert-Mumford stable.

More generally, let the Hilbert scheme $\mathcal{H}=\mathcal{H}(N, p)$ be the parameter space of subschemes of $\mathbf{C P}^{N}$ with the same Hilbert polynomial $p(k)$. The preceding procedure for associating an orbit of vectors $V_{\hat{X}}$ corresponds to a choice of an ample line bundle $\eta \rightarrow \mathcal{H}$, carrying a lift of the action of $S L(N+1)$. The orbit of vectors is then obtained by selecting a vector $V_{\hat{X}}$ in the fiber of $\eta$ over $\hat{X}$, and applying the $S L(N+1)$ action.

Definition 1. (a) Let $\eta \rightarrow \mathcal{H}$ be an ample line bundle over the Hilbert scheme equipped with a lifting of the $S L(N+1)$ action to $\eta$. Then a projective variety $\hat{X}$ is said to be $\eta$-stable if the orbit $\left\{\sigma \cdot V_{\hat{X}}\right\}$ is closed, and the stabilizer of $V_{\hat{X}}$ is finite.

(b) In the context of positive line bundles: Let $L \rightarrow X$ be an ample line bundle with Hilbert polynomial p. For $r>>1$, let $\mathcal{H}_{r}$ be the Hilbert scheme of subchemes of $\mathbf{C P}^{N_{r}}$ with Hilbert polynomial $p_{r}(k)=p(r k)$ and let $\eta_{r} \rightarrow \mathcal{H}_{r}$ be a family of ample line bundles with $S L\left(N_{r}+1\right)$ action. We say $(X, L)$ is $\eta_{r}$-stable if the image $\hat{X}=\iota_{\underline{\hat{s}}}(X) \subset \mathbf{C P}^{N_{r}}$ of $X$ by the Kodaira imbedding is $\eta_{r}$ stable. We say $(X, L)$ is asymptotically $\eta$ stable if it is $\eta_{r}$ stable for all sufficiently large $r$.

In general, the notion of stability in GIT depends on the choice of ample line bundle $\eta \rightarrow \mathcal{H}$. However, for Chow-Mumford and Hilbert-Mumford stability, we have the following recent theorem due to Mabuchi [93],

THEOREM 4. Let $L \rightarrow X$ be a positive line bundle over a compact complex manifold $X$. Then $L \rightarrow X$ is asymptotically Hilbert-Mumford stable if and only if $L \rightarrow X$ is asymptotically Chow-Mumford stable.

6.1.2. The Hilbert-Mumford criterion. There is a remarkable condition which is equivalent to stability in the sense of GIT, but which can be formulated without reference to the closures of orbits of vectors in $\mathbf{C}^{M}$ and is thus susceptible to generalization. This is the Hilbert-Mumford numerical criterion, which can be stated as follows.

Let $\hat{X} \in \mathcal{H}$, and let $\lambda: \mathbf{C}^{\times} \rightarrow S L(N+1)$ be a homomorphism. Set

$$
\hat{X}_{0}=\lim _{\tau \rightarrow 0} \lambda(\tau) \cdot \hat{X}
$$

Then $\hat{X}_{0}$ is fixed by $\lambda$, and, denoting $\lambda(\tau)$ as the lift of $\lambda$ to $\eta$, we define the numerical invariant $F$ by

$$
\lambda(\tau) \xi=\tau^{-F} \xi
$$

where $\xi$ is any non-zero vector in the fiber of $\eta$ above $\hat{X}_{0} \in \mathcal{H}$. Then the Hilbert-Mumford numerical criterion says that $\hat{X}$ is $\eta$-stable if and only if $F>0$ for all homomorphisms $\lambda: \mathbf{C}^{\times} \rightarrow S L(N+1)$. 
6.1.3. Chow-Mumford and K-stability. The Hilbert-Mumford criterion is a powerful tool, since it reduces matters to one-dimensional complex subgroups of $S L(N+1)$, and to the study of the sign of a single numerical invariant rather than the property of closure of orbits. Even more important for the problem of constant scalar curvature metrics, it can be used to define a notion of $\eta$-stability even when $\eta \rightarrow \mathcal{H}$ is a line bundle over the Hilbert scheme which is not necessarily ample.

Thus given a line bundle $\eta \rightarrow \mathcal{H}$, not necessarily ample, we define $L \rightarrow X$ to be $\eta$-stable if the corresponding numerical invariant $F$ defined by $(6.6)$ is strictly positive for all homomorphisms $\lambda: \mathbf{C}^{\times} \rightarrow S L(N+1)$.

We can now describe the notions of stability which arise in the context of metrics of constant scalar curvature. Each corresponds to a choice of line bundle $\eta \rightarrow \mathcal{H}$. They can be defined in several ways, but we shall give for each a formulation in terms of Deligne intersection pairings (a brief summary of basic facts about Deligne intersection pairings can be found in section $\S 6.1 .4)$. The advantage of the Deligne pairing formulation is that it provides a unified approach, and also ties in naturally with the $K$-energy and the functional $F_{\omega_{0}}^{0}$ which are central to the analytic formulation of the problem:

- Chow-Mumford stability: We have given earlier a geometric construction of the Chow-Mumford bundle. In view of a theorem of S. Zhang [166] (see also section $§ 9.1$ ), it can also be defined as the following Deligne intersection pairing,

$$
\eta_{\text {Chow }}=\langle O(1), \ldots, O(1)\rangle
$$

- K-stability: The line bundle $\eta_{K}$ defining the notion of $K$-stability can be defined in two equivalent ways, either in terms of the line bundles $\lambda_{j}=\lambda_{j}(L, X, \mathcal{H})$ of the Knudsen-Mumford expansion (see section $\S 6.1 .5$ below), or in terms of Deligne intersection pairings:

$$
\eta_{K}=\left\{\begin{array}{l}
\lambda_{n+1}^{\mu} \otimes\left(\frac{\lambda_{n+1}^{2}}{\lambda_{n}^{2}}\right)^{n+1} \\
\left\langle K_{X}, O(1), \ldots, O(1)\right\rangle^{n+1}\langle O(1), \ldots, O(1)\rangle^{n c_{1}(X) c_{1}(L)^{n-1} / c_{1}(L)^{n}}
\end{array}\right.
$$

This algebraic notion of $K$-stability is due to Donaldson [47], and is very similar to an earlier analytic notion of $K$-stability due to Tian $[\mathbf{1 4 3 , 1 4 5}$, who defined it in terms of a Futaki invariant for special degenerations with $Q$-Fano (and in particular, normal) central fibers (see $\S 7.1$ ). Both notions of $K$-stability have been conjectured to be equivalent to the existence of a constant scalar curvature Kähler metric in $c_{1}(L)$, the analytic version in $[\mathbf{1 4 3}]$, and the algebraic version in $[\mathbf{4 7}]$.

The equivalence between Donaldson's original formulation and the formulation in terms of Knudsen-Mumford expansions is in [100]. The equivalence with the above Deligne intersection pairing formulation is in [102]. 
A slightly stronger version of $K$-stability has been proposed by Szekelyhidi [136]. Another version in terms of slopes, and hence more similar to the notion of Mumford-Takemoto stability for vector bundles, has been introduced by Ross and Thomas [118].

6.1.4. Deligne pairings and energy functionals. We provide here a summary of Deligne pairings and explain how they are related to energy functionals $[166,107,130]$.

Let $L_{0}, \ldots, L_{n}$ be holomorphic line bundles over a complex manifold $X$ of dimension $\operatorname{dim} X=n$. Then the Deligne pairing $\left\langle L_{0}, L_{1}, \ldots, L_{n}\right\rangle$ is a one-dimensional space, generated by the symbol $\left\langle l_{0}, l_{1}, \ldots, l_{n}\right\rangle$, where $l_{i}$ are generic meromorphic sections of $L_{i}$ in general position, transforming in the following way under changes of sections $l_{i} \rightarrow l_{i}^{\prime}$,

$$
\left\langle l_{0}^{\prime}, l_{1}, \ldots, l_{n}\right\rangle=\left(\prod_{z \in \cap_{i \neq 0} \operatorname{div} l_{i}} \frac{l_{0}^{\prime}(z)}{l_{0}(z)}\right)\left\langle l_{0}, l_{1}, \ldots, l_{n}\right\rangle .
$$

More generally, if $\pi: X \rightarrow B$ is a flat projective morphism of integral schemes of pure relative dimension $n$, and $L_{0}, L_{1}, \ldots, L_{n}$ are line bundles over $X$, then we can define the Deligne pairing

$$
\left\langle L_{0}, L_{1}, \ldots, L_{n}\right\rangle(X / B)
$$

which is a line bundle on $B$, locally generated by symbols $\left\langle l_{0}, l_{1}, \ldots, l_{n}\right\rangle$ with $l_{j}$ rational sections of $L_{j}$ in general position and transforming as above under changes of sections $l_{j}$. A useful property is the induction formula

$$
\left\langle L_{0}, \ldots, L_{n-1}, L_{n}\right\rangle(X / B)=\left\langle L_{0}, \ldots, L_{n-1}\right\rangle\left(\operatorname{div} l_{n} / B\right),
$$

if all components of $\operatorname{div} l_{n}$ are flat over $B$.

A key feature of Deligne pairings is that they are equipped with a natural metric $\left\langle h_{0}, \ldots, h_{n}\right\rangle$, if each line bundle $L_{j}$ comes equipped with a metric $h_{j}$. This metric satisfies the following property

$$
\left\langle h_{0} e^{-\phi}, h_{1}, \ldots, h_{n}\right\rangle=e^{-\psi}\left\langle h_{0}, h_{1}, \ldots, h_{n}\right\rangle
$$

where $\psi: B \rightarrow \mathbf{C}$ is the function

$$
\psi=\int_{X / B} \phi \cdot \prod_{j=1}^{n} \omega_{j}\left(L_{j}\right)
$$

and $\omega_{j}\left(L_{j}\right) \equiv-\frac{i}{2} \partial \bar{\partial} \log h_{j}$ is the curvature of $h_{i}$. Denoting by $O(f)$ the trivial bundle with metric $\|1\|=e^{-f}$, we obtain the following induction formula with metrics

$$
\begin{aligned}
& \left\langle L_{0}, \ldots, L_{n-1}, L_{n}\right\rangle(X / B) \\
& \quad=\left\langle L_{0}, \ldots, L_{n-1}\right\rangle\left(\operatorname{div} l_{n} / B\right) \otimes O\left(-\int_{X / B} \log \left\|l_{n}\right\| \wedge_{j=0}^{n-1} \omega_{j}\left(L_{j}\right)\right)
\end{aligned}
$$

The lines bundles $\eta_{C h o w}$ and $\eta_{K}$ defining Chow-Mumford and $K$-stability can now be related to the functionals $F_{\omega_{0}}^{0}$ and $K_{\omega_{0}}$ by $[\mathbf{1 0 7}, \mathbf{1 1 0}]$. 
TheOREm 5. Let $L \rightarrow X$ be a positive holomorphic line bundle, and let $\eta_{\text {Chow }}$ and $\eta_{K}$ be given by the Deligne pairings (6.7) and (6.8). For each metric $h_{0}$ on $L$, let $\omega_{0}=-\frac{i}{2} \partial \bar{\partial} \log h_{0}$ be the corresponding metric on $X$, and equip $\eta_{C h o w}$ and $\eta_{K}$ with the corresponding metrics as Deligne pairings. Then under a change of metric $h \rightarrow h e^{-\phi}$, we have

$$
\begin{aligned}
\eta_{\text {Chow }}\left(h_{0} e^{-\phi}\right) & =\eta_{\text {Chow }}\left(h_{0}\right) \otimes O\left((n+1) c_{1}(L)^{n} F_{\omega_{0}}^{0}(\phi)\right) \\
\eta_{K}\left(h_{0} e^{-\phi}\right) & =\eta_{K}\left(h_{0}\right) \otimes O\left(-(n+1) c_{1}(L)^{n} K_{\omega_{0}}^{0}(\phi)\right)
\end{aligned}
$$

These basic relations provide yet additional evidence that the functionals $F_{\omega_{0}}^{0}(\phi)$ and $K_{\omega_{0}}(\phi)$ are closely related to the algebraic-geometric notion of stability. Similarly, the Futaki functional defined by Futaki in [58] can also be realized in terms of Deligne pairings, more specifically $\left\langle K_{X}^{-1}, K_{X}^{-1}, \ldots, K_{X}^{-1}\right\rangle$. For the relation of Deligne pairings to other functionals in Kähler geometry, see [130]. At the 2002 Complex Geometry conference in Tokyo, Professors T. Mabuchi and L. Weng have informed us that they have also been aware of potential applications of Deligne pairings to Kähler geometry for some time.

6.1.5. Deligne pairings and Knudsen-Mumford expansions. We discuss now the two formulations of $\eta_{K}$ given in (6.8) and their equivalence.

Let $\pi: X \rightarrow B$ be again a morphism of flat integral schemes with constant relative dimension, and let $L \rightarrow X$ be a relatively ample line bundle. The theorem of Knudsen-Mumford [77] says that there exist functorially defined line bundles $\lambda_{j}=\lambda_{j}(X, L, B) \rightarrow B$ such that the following isomorphism holds for all $k>>1$

$$
\operatorname{det} \pi_{*}\left(L^{k}\right) \sim \lambda_{n+1}^{\left(\begin{array}{c}
k \\
n+1
\end{array}\right)} \otimes \lambda_{n}^{\left(\begin{array}{c}
k \\
n
\end{array}\right)} \otimes \cdots \otimes \lambda_{0} .
$$

Deligne [39] showed that, in dimension $n=1$, we have $\lambda_{2}=\langle L, L\rangle_{\mathcal{X} / B}$ and $\lambda_{1}^{2}=\left\langle L K_{X}^{-1}, L\right\rangle_{X / B}$. More generally, for general $n$, Knudsen-Mumford showed that $\lambda_{n+1}$ is equal to the Chow bundle. Combined with the theorem of Zhang [166], which says that the Chow bundle is in turn equal to $\langle L, \ldots, L\rangle_{X / B}$ (see Theorem 19 below), we have then

$$
\lambda_{n+1}(L, X, B)=\langle L, \ldots, L\rangle_{X / B} .
$$

The bundle $\eta_{K}$ in terms of the Knudsen-Mumford line bundles $\lambda_{j}$ in the first line of the definition (6.8) was given by Paul-Tian [100], who pointed out that its numerical invariants coincided with the Donaldson-Futaki invariants defined by Donaldson [47] in his definition of $K$-stability. The bundle $\eta_{K}$ in terms of Deligne pairings in the second line of the definition (6.8) was first introduced in $[\mathbf{1 0 7}, \mathbf{1 1 0}]$. The following theorem due to $[\mathbf{1 0 2}]$ identifies 
the second leading term $\lambda_{n}$ in the Knudsen-Mumford expansion, and shows that the two bundles in the definition of $\eta_{K}$ in (6.8) are equivalent:

Theorem 6. Let $\pi: X \rightarrow B$ be a proper flat morphism of integral schemes of relative dimension $n \geq 0$, and let $L \rightarrow X$ which is very ample on the fibers. Assume that $X$ and $B$ are smooth. Let $K$ be the relative canonical line bundle of $X \rightarrow B$. Then

(i) There is a canonical functorial isomorphism

$$
\lambda_{n}^{2}(L, X, B)=\left\langle L^{n} K^{-1}, \ldots, L\right\rangle_{X / B} .
$$

(ii) In particular,

$$
\lambda_{n+1}^{\mu} \otimes\left(\frac{\lambda_{n+1}^{2}}{\lambda_{n}^{2}}\right)^{n+1}=\left\langle K_{X}, O(1), \ldots, O(1)\right\rangle^{n+1}\langle O(1), \ldots, O(1)\rangle^{\frac{n c_{1}(X) c_{1}(L)^{n-1}}{c_{1}(L)^{n}}}
$$

6.1.6. Test configurations and Donaldson-Futaki invariants. As we have seen in $\$ 6.1 .3$, the definition of $\eta$-stability, when $\eta$ is not ample, consists solely in the requirement that the corresponding numerical invariant $F$ be strictly positive. Thus it is useful to exhibit this numerical invariant more concretely in the case of $K$-stability. For this as well as for other subsequent uses, it is preferable to use the formalism of "test configurations" introduced by Donaldson [47] rather than of one-parameter subgroups. In view of its importance, we describe this formalism in some detail in this section.

Definition 2. Let $L \rightarrow X$ be a positive line bundle over a compact complex manifold $X$. A test configuration $\mathcal{T}$ consists of

(1) a scheme $\mathcal{X}$ with a $\mathbf{C}^{\times}$action $\rho$,

(2) an $\mathbf{C}^{\times}$equivariant line bundle $\mathcal{L} \rightarrow \mathcal{X}$, ample on all fibers,

(3) and a flat $\mathbf{C}^{\times}$equivariant map $\pi: \mathcal{X} \rightarrow \mathbf{C}$ where $\mathbf{C}^{\times}$acts on $\mathbf{C}$ by multiplication, with the following properties: the pair $\left(X_{1}, L_{1}\right)$ is isomorphic to $\left(X, L^{r}\right)$ for some $r>0$ and $L^{r}$ is very ample. Here $X_{w}=\pi^{-1}(w)$ and $L_{w}=\mathcal{L}_{\left.\right|_{X_{w}}}$.

It is convenient to denote the test configuration $\mathcal{T}$ by

$$
\mathcal{T}=\left(\rho: \mathbf{C}^{\times} \rightarrow \operatorname{Aut}(\mathcal{L} \rightarrow \mathcal{X} \rightarrow \mathbf{C})\right) .
$$

We also say that a test configuration is trivial if $\mathcal{L}=L \times \mathbf{C}$ with the trivial action $\rho(\tau)(l, w)=(l, \tau w)$, for $(l, w) \in L \times \mathbf{C}, \tau \in \mathbf{C}^{\times}$.

An important property of a test configuration $\mathcal{T}$ is that it defines for each $k$ an $\left(N_{k}+1\right) \times\left(N_{k}+1\right)$ diagonal matrix $B_{k}$ and its traceless part $A_{k}$. Indeed, in a test configuration $\mathcal{T}$, the fiber $L_{0} \rightarrow X_{0}$ is fixed by the $\mathbf{C}^{\times}$ action $\rho$. Thus we obtain an induced automorphism $\rho_{k}(\tau)$ of $H^{0}\left(X_{0}, L_{0}^{k}\right)$

$$
\rho_{k}(\tau): H^{0}\left(X_{0}, L_{0}^{k}\right) \longrightarrow H^{0}\left(X_{0}, L_{0}^{k}\right)
$$

and hence, if we choose a basis $\underline{s}$ of $H^{0}\left(X_{0}, L_{0}^{k}\right)$, we obtain a one-parameter subgroup $\rho: \mathbf{C}^{\times} \rightarrow G L\left(N_{k}+1\right)$. Since every one-parameter subgroup 
is a direct sum of one dimensional subgroups, there exists a basis $\underline{s}$ with the following property. The matrix $\rho(\tau)$ is diagonal with entries $\tau^{\lambda_{\alpha}}$ where $\lambda_{0}^{(k)} \leq \cdots \leq \lambda_{N_{k}}^{(k)}$ are integers that are independent of $\tau$. We let $B_{k}$ be the diagonal matrix with entries $\lambda_{\alpha}^{(k)}$ and we shall often write

$$
\rho(\tau)=\tau^{B_{k}}
$$

The matrix $A_{k}$ is then defined to be the traceless part of $B_{k}$.

We shall see shortly (c.f. Lemma 1 below) that a test configuration can be imbedded equivariantly into $\mathbf{C P}^{N_{k}}$, with $\tau^{B_{k}}$ defining a one-parameter subgroup completed by the central fiber $X_{0}$ (6.5). Thus, for each choice of bundle $\eta$ over the Hilbert scheme, as in the case of one-parameter subgroups described in section $\S 6.1 .2$, we can define a numerical invariant. Since we had not given any detail there of the construction of the numerical invariant, we do so now, in the equivalent context of test configurations.

Let as before $p(k) \equiv \operatorname{dim} H^{0}\left(X, L^{k}\right)$ be the Hilbert polynomial of $L \rightarrow X$, and let $\mathcal{H}$ be the Hilbert scheme of all subvarieties of $\mathbf{C P}^{p(k)-1}$ with Hilbert polynomial $p(k)$. Let $\eta \rightarrow \mathcal{H}$ be a line bundle on $\mathcal{H}$, not necessarily ample, which carries a linearization of $G L(p(k), \mathbf{C})$, that is, an action of $G L(p(k), \mathbf{C})$ which is a lift of the natural action of $G L(p(k), \mathbf{C})$ on $\mathcal{H}$.

Let $\mathcal{T}=\left(\rho: \mathbf{C}^{\times} \rightarrow \operatorname{Aut}(\mathcal{L} \rightarrow \mathcal{X} \rightarrow \mathbf{C})\right)$ be a test configuration. Then we have seen that $\mathcal{T}$ induces an endomorphism $\rho_{k}(\tau)$ on $H^{0}\left(X_{0}, L_{0}^{k}\right)$ defined by

$$
\left(\rho_{k}(\tau)(s)\right)(x)=\rho(\tau)^{-1}(s(\rho(\tau) x)) \text { for all } x \in X_{0}
$$

so if we fix a basis $\underline{s}=\left(s_{0}, \ldots, s_{p(k)-1}\right)$ then $\rho(\tau)\left(s_{\alpha}\right)=\sum \sigma(\tau)_{\alpha \beta} s_{\beta}$ for some invertible matrix $\sigma(\tau)=\left(\sigma(\tau)_{\alpha \beta}\right) \in G L(p(k))$. Moreover, $\sigma: \mathbf{C}^{\times} \rightarrow$ $G L(p(k))$ is a one parameter subgroup. For $k>>1$ we let $\iota_{\underline{s}}: X_{0} \hookrightarrow$ $\mathbf{C P}^{p(k)-1}$ be the imbedding $x \mapsto\left[s_{0}(x), \ldots, s_{p(k)-1}(x)\right]$. Then one easily sees that $\iota_{\underline{s}}\left(X_{0}\right)$ is invariant under the action of $\sigma(\tau)$. Indeed, if $x \in X_{0}$ then $\rho(\tau) x \in X_{0}$ and

$$
\sigma(\tau)\left(\iota_{\underline{s}}(x)\right)=\left(\iota_{\underline{s}}(\rho(\tau) x)\right)
$$

To see this we observe

$$
\begin{aligned}
\sigma(\tau)\left(\iota_{\underline{s}}(x)\right) & =\left(\rho_{k}(\tau)\left(s_{\alpha}\right)\right)(x)=\left(\rho(\tau)^{-1}\left(s_{\alpha}(\rho(\tau) x)\right)\right. \\
& =\frac{s_{\alpha}(\rho(\tau) x)}{s_{\alpha_{0}}(\rho(\tau) x)} \rho(\tau)^{-1}\left(s_{\alpha_{0}}(\rho(\tau) x)\right)=\left(s_{\alpha}(\rho(\tau) x)\right)
\end{aligned}
$$

Now let $\left[\iota_{s}\right] \in \mathcal{H}$ be the point in the Hilbert scheme corresponding to the imbedding $\iota_{s}: X_{0} \hookrightarrow \mathbf{C P}^{p(k)-1}$. The discussion above shows that $\left[\iota_{s}\right]$ is a fixed point for the action of $\sigma(\tau) \in G L(p(k))$. Since the $G L(p(k), \mathbf{C})$ action on the Hilbert scheme $\mathcal{H}$ lifts to an action on $\eta$, it follows that there exists $F \in \mathbf{Z}$ such that

$$
\sigma(\tau) n_{\underline{s}}=\tau^{-F} n_{\underline{s}}
$$

for all $n_{\underline{s}}$ in the fiber of $\eta$ above $\left[\iota_{\underline{s}}\right] \in \mathcal{H}$. 
We claim that $F$ is independent of the basis $\underline{s}$. To see this, let $\underline{\tilde{s}}$ be another basis and $\gamma \in G L(p(k))$ chosen so that $\underline{\tilde{s}}=\gamma \underline{s}$. Let $\tilde{\sigma}(\tau)$ be the matrix representing $\rho_{k}(\tau)$ with respect to the basis $\underline{\tilde{s}}$. Then $\tilde{\sigma}(\tau)=\gamma \sigma(\tau) \gamma^{-1}$. Moreover $\gamma \iota_{\underline{s}}(x)=\iota_{\underline{s}}(x)$ so $\gamma\left[\iota_{\underline{s}}\right]=\left[\iota_{\underline{\tilde{s}}}\right]$. Fix $n_{\underline{s}}$ as above and define $n_{\underline{\tilde{s}}}=\gamma n_{\underline{s}}$. Then

$$
\tilde{\sigma}(\tau) n_{\underline{\tilde{s}}}=\gamma \sigma(\tau) \gamma^{-1} \gamma n_{\underline{s}}=\gamma \tau^{-F} n_{\underline{s}}=\tau^{-F} n_{\underline{\tilde{s}}}
$$

establishing the desired invariance.

The bundle $L \rightarrow X$ is consequently defined to be $\eta$-stable if $F>0$ for all test configurations. In view of the correspondence between testconfigurations and one-parameter subgroups given below, this definition is just a rephrasing in terms of test configurations of the earlier definition of $\eta$ stability. However, it allows us to make now contact, in the case of $\eta=\eta_{K}$, with the original definition of $K$-stability by Donaldson [47]:

Definition 3. Let $X_{0}$ be a projective scheme and $\alpha: \mathbf{C}^{\times} \rightarrow \operatorname{Aut}\left(L_{0} \rightarrow\right.$ $\left.X_{0}\right)$ an algebraic homomorphism. Then $F(\alpha)$ is defined by the asymptotic expansion $^{2}$

$$
\frac{\text { Trace } B_{k}}{k\left(N_{k}+1\right)}=F_{0}-F(\alpha) k^{-1}+O\left(k^{-2}\right)
$$

where $B_{k}: H^{0}\left(X_{0}, L_{0}^{k}\right) \rightarrow H^{0}\left(X_{0}, L_{0}^{k}\right)$ is the infinitesimal generator of the one-parameter group of endomorphisms induced by the action of $\alpha$.

Now let $L \rightarrow X$ be an ample line bundle. Let $\mathcal{T}$ be a test configuration for $L \rightarrow X$. Then the Donaldson-Futaki invariant $F(\mathcal{T})$ is defined by $F(\mathcal{T})=$ $F(\tilde{\rho})$ where $\tilde{\rho}$ is the restriction of $\rho$ to the central fiber.

In [47], Donaldson defined $L \rightarrow X$ to be $K$-stable if $F(\mathcal{T})>0$ for any non-trivial test configuration $\mathcal{T}$. As we had mentioned earlier, it was noted by Paul-Tian $[\mathbf{1 0 0}]$ that $F(\mathcal{T})$ coincided with the numerical invariant $F$ of the bundle $\eta_{K}$. Thus the definition of $K$-stability of [47] coincides with $\eta_{K}$-stability as defined in section $§ 6.1 .3$.

6.1.7. Equivariant imbeddings of test configurations. The correspondence between one-parameter subgroups and test configurations is described by the following lemma, which provides an equivariant imbedding of test configurations into projective space (see the original statement in [50], and [114] for the version presented here):

Lemma 1. (i) Let $X$ be a subvariety of $\mathbf{C P}^{N_{k}}$ and let $\lambda: \mathbf{C}^{\times} \rightarrow G L$ $\left(N_{k}+1\right)$ be a one-parameter subgroup of $G L\left(N_{k}+1\right)$. Let

$$
\mathcal{X}^{\times}=\left\{(\lambda(\tau) x, \tau): \tau \in \mathbf{C}^{\times}, x \in X\right\} \subseteq \mathbf{C P}^{N_{k}} \times \mathbf{C}^{\times} .
$$

Then the closure (or, more precisely, the flat limit) of $O(1) \times \mathbf{C}^{\times} \rightarrow \mathcal{X}^{\times} \rightarrow$ $\mathbf{C}^{\times}$inside $O(1) \times \mathbf{C} \rightarrow \mathbf{P}^{N_{k}} \times \mathbf{C} \rightarrow \mathbf{C}$, is a test configuration.

\footnotetext{
${ }^{2}$ Our conventions for $F(\alpha)$ differ from those of $[\mathbf{4 7}]$ by a minus sign.
} 
(ii) Conversely, let $L \rightarrow X$ be a positive line bundle, and $\rho: \mathbf{C}^{\times} \rightarrow$ $\operatorname{Aut}(\mathcal{L} \rightarrow \mathcal{X} \rightarrow \mathbf{C})$ be a test configuration. Let $k$ be an integer such that $L^{k}$ is very ample. Let $B_{k}$ be the matrix defined in (6.22). Then there exists a basis $\underline{s}$ of $H^{0}\left(X, L^{k}\right)$, and an imbedding

$$
I_{\underline{s}}:\left(\mathcal{L}^{k} \rightarrow \mathcal{X} \rightarrow \mathbf{C}\right) \rightarrow\left(O(1) \times \mathbf{C} \rightarrow \mathbf{C P}^{N_{k}} \times \mathbf{C} \rightarrow \mathbf{C}\right)
$$

which restricts to $\iota_{\underline{s}}$ on the fiber $X_{1}$ and intertwines $\rho(\tau)$ and $B_{k}$, i.e.,

$$
I_{\underline{s}}\left(\rho(\tau) l_{w}\right)=\left(\tau^{B_{k}} \cdot I_{\underline{s}}\left(l_{w}\right), \tau w\right) \quad \text { for each } l_{w} \in L^{k}
$$

(iii) If $h$ is a metric on $L$ with positive curvature $\omega=-\frac{i}{2} \partial \bar{\partial} \log h$, then we may choose $\underline{s}$ to be an orthonormal basis of $H^{0}\left(X, L^{k}\right)$ with respect to the $L^{2}$ metric on $H^{0}\left(X, L^{k}\right)$ induced by $h$ and $\omega$. Moreover, the basis $\underline{s}$ is unique up to $U\left(N_{k}+1\right)$ elements commuting with $B_{k}$.

6.1.8. Bott-Chern secondary characteristic classes. We have seen how the energy functionals $F_{\omega}^{0}(\phi)$ and $K_{\omega}(\phi)$ are readily related to Deligne intersection pairings, and whence to Chow-Mumford and $K$-stability. There is an alternative construction of energy functionals, based on Bott-Chern secondary characteristic classes, which is also of interest. It began with Donaldson's [43] construction of the functional for Hermitian-Einstein metrics, and has been developed further by Tian $[\mathbf{1 4 4}]$ in a more general context. We give a brief exposition following [144].

Let $E \rightarrow X$ be a holomorphic vector bundle of rank $r$ over a compact Kähler manifold $X$. For each function $\Phi(A)$ on $g l(r, \mathbf{C})$ which is a symmetric polynomial of degree $k$ in the eigenvalues of $A$, we have a Chern-Weil $(k, k)$-form $\Phi\left(\frac{i}{2 \pi} F(H)\right)$, defined for each Hermitian metric $H_{\bar{\alpha} \beta}$ on $E$, where $F=-\bar{\partial}\left(H^{-1} \partial H\right)$ is the curvature of $H$. These forms are cohomologically equivalent for different metrics $H_{\bar{\alpha} \beta}$. In fact, we can write explicitly

$$
\Phi\left(\frac{i}{2 \pi} F(H)\right)-\Phi\left(\frac{i}{2 \pi} F\left(H^{\prime}\right)\right)=-\partial \bar{\partial} \mathbf{B C}\left(\Phi ; H, H^{\prime}\right),
$$

where $\mathbf{B C}$ is a form given explicitly modulo $\operatorname{Im} \partial+\operatorname{Im} \bar{\partial}$ by

$$
\mathbf{B C}\left(\Phi ; H, H^{\prime}\right)=\int_{0}^{1} \Phi^{\prime}\left(\frac{i}{2 \pi} F\left(H_{t}\right), H_{t}^{-1} \dot{H}_{t}\right) d t .
$$

Here $H_{t}$ is a smooth one-parameter family of metrics joining $H=H_{0}$ to $H^{\prime}=$ $H_{1}$, and the derivative $\Phi^{\prime}$ of $\Phi$ is defined by $\Phi^{\prime}(A ; B)=\left.\frac{d}{d t}\right|_{t=0}\left(A e^{t B}\right)$. The form $\mathbf{B C}$ is called the Bott-Chern secondary characteristic class associated to $\Phi$. The corresponding Donaldson functional is then defined by

$$
D\left(\Phi ; H, H^{\prime}\right)=\int_{X} \mathbf{B C}\left(\Phi ; H, H^{\prime}\right) .
$$

To make connection with the functionals $F_{\omega_{0}}^{0}(\phi)$ and $K_{\omega_{0}}(\phi)$ introduced in the previous sections, let $L$ be now a positive line bundle over $X$ with metric 
$h_{0}$ and positive curvature $\omega_{0}$. If we choose $\Phi(A)=\operatorname{Trace}\left(A^{n+1}\right)$, then for any other metric $e^{-\phi} h_{0}$ with positive curvature $\omega_{\phi}=\omega_{0}+\frac{i}{2} \partial \bar{\partial} \phi$, we have

$$
F_{\omega_{0}}^{0}(\phi)=D\left(\Phi ; h_{0}, e^{-\phi} h_{0}\right) .
$$

Similarly, $K_{\omega_{0}}(\phi)$ coincides with $D\left(\Phi ; H, H^{\prime}\right)$, when $E$ is the virtual bundle defined by

$$
E=(n+1)\left(K_{X}^{-1}-K_{X}\right) \otimes\left(L-L^{-1}\right)^{n}-\mu\left(L-L^{-1}\right)^{n+1} .
$$

6.2. Donaldson's infinite-dimensional GIT. Beginning in the late 1990's, Donaldson [44] developed an approach to the problem of constant scalar curvature Kähler metrics, motivated partly by the interpretation of the scalar curvature as the moment map of an infinite-dimensional group of symplectic automorphisms. Central to this approach is a natural interpretation of the space $\mathcal{K}$ of Kähler potentials ${ }^{3}$ in $c_{1}(L)$,

$$
\mathcal{K}=\left\{\phi \in C^{\infty}(X) ; \omega_{0}+\frac{i}{2} \partial \bar{\partial} \phi>0\right\}
$$

as an infinite-dimensional symmetric space, whose geodesics would play a similar role to one-parameter subgroups in the usual finite-dimensional GIT.

The starting point is that $\mathcal{K}$ admits a natural Riemannian metric, given at $\phi$ by

$$
\|\delta \phi\|_{\phi}^{2}=\int_{X}|\delta \phi|^{2} \omega_{\phi}^{n}
$$

where we have identified the tangent space to $\mathcal{K}$ at $\phi$ with $C^{\infty}(X)$. The following theorem is then due to Donaldson [44], Semmes [120], and Mabuchi [91]:

TheOREM 7. The Riemannian manifold $\mathcal{K}$ is an infinite-dimensional symmetric space of non-positive curvature, in the sense that its curvature is covariant constant and non-positive. In fact, the Riemann curvature tensor $R_{\phi}$ and the sectional curvature $K_{\phi}$ at the point $\phi \in \mathcal{K}$ are given by the following expression

$$
\begin{aligned}
R_{\phi}\left(\delta_{1} \phi, \delta_{2} \phi\right) \delta_{3} \phi & =-\frac{1}{4}\left\{\left\{\delta_{1} \phi, \delta_{2} \phi\right\}_{\phi}, \delta_{3} \phi\right\}_{\phi}, \\
K_{\phi}\left(\delta_{1} \phi, \delta_{2} \phi\right) & =-\frac{1}{4}\left\|\left\{\delta_{1} \phi, \delta_{2} \phi\right\}_{\phi}\right\|_{\phi}^{2},
\end{aligned}
$$

where $\{,\}_{\phi}$ is the Poisson bracket on $C^{\infty}(X)$ defined by the symplectic form $\omega_{\phi}$.

It is also shown in $[\mathbf{2 1}]$ that the space $\mathcal{K}$ is non-positive in the sense of Alexandrov, and essentially that the Calabi flow (see §10.1) is distance decreasing in this space.

\footnotetext{
${ }^{3}$ We denote the space of Kähler potentials and the space of corresponding Kähler metrics by the same letter $\mathcal{K}$, although strictly speaking, the latter is equal to the former modulo constants.
} 
6.2.1. Geodesic segments and geodesic rays. The geodesics $(-T, 0] \ni t \rightarrow$ $\phi(t)$ in $\mathcal{K}$ are by definition the solutions of the Euler-Lagrange equation of the length functional. They are readily seen to be given by

$$
\ddot{\phi}-g_{\phi}^{j \bar{k}} \partial_{j} \dot{\phi} \partial_{\bar{k}} \dot{\phi}=0
$$

where we have denoted by $\left(g_{\phi}\right)_{\bar{k} j}=g_{\bar{k} j}^{0}+\partial_{j} \partial_{\bar{k}} \phi$ the metric corresponding to the Kähler form $\omega_{\phi}$. The geodesic equation admits an interpretation as a completely degenerate complex Monge-Ampère equation which will be crucial in subsequent developments: let $A=\left\{w \in \mathbf{C} ; e^{-T} \leq|w| \leq 1\right\}$ and define the $S^{1}$ invariant function $\Phi(z, w)$ on $X \times A$ as in $(5.17)$ by $\Phi(z, w)=$ $\phi(z, \log |w|)$. Let $\Omega_{0}$ be again the Kähler form $\omega_{0}$ viewed as a form on $X \times A$. Then in view of the equation (5.20), we see that the above geodesic equation is equivalent to the following completely degenerate Monge-Ampère equation $[\mathbf{1 2 0}, 44]$

$$
\left(\Omega_{0}+\frac{i}{2} \partial \bar{\partial} \Phi\right)^{n+1}=0 \text { on } X \times A .
$$

Geodesic segments with given end points correspond to $S^{1}$ invariant solutions of the degenerate complex Monge-Ampère equation with given Dirichlet boundary conditions on $X \times \partial A$. We shall also be particularly interested in geodesic rays, which would be defined for $t \in(-\infty, 0]$, and which correspond to the same set-up with $A$ replaced by the punctured disk $D^{\times}=$ $\{w \in \mathbf{C} ; 0<|w| \leq 1\}$. In the case of a given starting point for the geodesic ray, the Dirichlet boundary condition would be specified at $X \times\{|w|=1\}$. The formulation in terms of the complex degenerate Monge-Ampère equation also provides us with a natural notion of generalized geodesics: these will be by definition $S^{1}$ invariant, locally bounded, generalized solutions of the Monge-Ampère equation (6.41) in the sense of pluripotential theory (see $\S 12.3 .1)$.

6.2.2. Stability conditions in terms of geodesic rays. We have seen that, by the Tian-Yau-Zelditch theorem, the space $\mathcal{K}$ of Kähler potentials can be viewed as the limit of the spaces $\mathcal{K}_{k}$ as $k \rightarrow \infty$. Now stability conditions are conditions on the numerical invariants of one-parameter orbits of $\mathcal{K}_{k}$. Since $\mathcal{K}_{k}$ is a symmetric space, these are the same as geodesics, and it is then natural to construct a GIT theory based directly on geodesic rays in the infinite-dimensional symmetric space $\mathcal{K}$.

The cost of working in an infinite-dimensional setting is partly offset by the intuition we gain from the theory of symmetric spaces and moment maps [44]. There is also a very powerful incentive for the use of geodesic rays in $\mathcal{K}$ : it is the fact that the $K$-energy is convex along these rays. In fact, if $\phi(t)$ is any smooth path in $\mathcal{K}$, then we have

$$
\ddot{K}_{\omega}(\phi(t))=\frac{1}{V} \int_{X}|\bar{\nabla} \bar{\nabla} \dot{\phi}|^{2} \omega_{\phi}^{n}-\frac{1}{V} \int\left(\ddot{\phi}-g_{\phi}^{j \bar{k}} \partial_{j} \dot{\phi} \partial_{\bar{k}} \dot{\phi}\right)(R-\mu n) \omega_{\phi}^{n} .
$$


This implies that $K_{\omega}(\phi(t))$ is convex when $\phi(t)$ is a geodesic, and in fact, strictly convex if $\operatorname{Aut}^{0}(X)=0$. This strict convexity has some immediate consequences: if geodesics always exist, the critical points, and thus the metrics of constant scalar curvature, would be unique.

The analogy with stability in GIT leads to the following early conjecture/question of Donaldson [44]: the non-existence of constant scalar curvature Kähler metrics in $c_{1}(L)$ should be equivalent to the existence of an infinite geodesic ray $\phi_{t}, t \in(-\infty, 0]$ such that

$$
\frac{1}{V} \int_{X} \dot{\phi}(R-\mu n) \omega_{\phi}^{n}<0
$$

for all $t \in(-\infty, 0]$. Since the left-hand side is just $-\dot{K}_{\omega}(\phi)$ at $\phi_{t}$, this condition is very close to the formulation of stability in terms of the asymptotic behavior of energy functionals, and can be viewed as an infinite-dimensional version of $K$-stability.

6.3. Stability conditions on $\operatorname{Diff}(X)$ orbits. There is another concept of stability which arises naturally if we try to construct the moduli space of complex structures on a smooth manifold $X$ as the space of equivalence classes of integrable almost-complex structures $J$ on $X$, modulo diffeomorphisms

$$
\{J \text { integrable almost - complex structure }\} / \operatorname{Diff}(X) .
$$

A point in this moduli space would then be an orbit $\operatorname{Diff}(X) \cdot J$ for some $J$, and the orbit would have to be closed in order for the point to be a Hausdorff point. From this point of view, the closedness of orbits is a condition similar to the ones in stability in GIT, except that the orbits are now that of the infinite-dimensional group $\operatorname{Diff}(X)$ rather than the finite-dimensional groups $S L(N+1)$. We describe now some specific versions of such stability conditions, motivated as we shall see by the convergence of the Kähler-Ricci flow $([\mathbf{1 1 2}, \mathbf{1 0 5}]$, and section $\S 8.2)$.

6.3.1. Condition (B). Let $X$ be a compact Kähler manifold and let $J$ be the almost-complex structure of $X$, viewed as a tensor. Condition (B) is the following condition $[\mathbf{1 1 2}]$ :

(B): There exists no almost-complex structure $\tilde{J}$ in the $C^{\infty}$ closure of the orbit $\operatorname{Diff}(X) \cdot J$ with

$$
\operatorname{dim} H^{0}\left(X, T_{\tilde{J}}^{1,0}\right)>\operatorname{dim} H^{0}\left(X, T_{J}^{1,0}\right)
$$

Here $H^{0}\left(X, T_{\tilde{J}}^{1,0}\right)$ denotes the space of holomorphic $(1,0)$ vector fields with respect to the integrable almost-complex structure $\tilde{J}$.

Clearly, if Condition (B) is violated, the orbit Diff $(X) \cdot J$ cannot be closed, and thus does not define a Hausdorff point.

For our purposes (see section $§ 8.2 .4$ ), we actually do not need the full strength of condition (B), but actually only the following weaker version 
which may be closer in spirit to the other stability conditions formulated in this chapter:

$\left(\mathrm{B}^{*}\right)$ : Let $g_{m}$ be any sequence of metrics in $c_{1}(X)$ with $K$-energy bounded from above, and which converges in $C^{\infty}$ in the sense of Cheeger-Gromov, i.e., there is a family of diffeomorphisms $F_{m}: X \rightarrow X$ so that $F_{m}^{*}\left(g_{m}\right)$ converges in $C^{\infty}$. Then the sequence of almost-complex structures $F_{m}^{*}(J)$ does not admit any $C^{\infty}$ limit point $J_{\infty}$ satisfying (6.45).

6.3.2. Condition $(S)$. A second type of stability condition that arises in the study of the Kähler-Ricci flow is a condition called there Condition (S). Let $X$ be a compact Fano manifold and let $J$ be again the almost-complex structure of $X$, viewed as a tensor. Condition $(\mathrm{S})$ is the following condition $[\mathbf{1 0 5}, \mathbf{1 0 6}]$ :

$(\mathrm{S})$ : There exists some solution $\omega(t)$ to the Kähler-Ricci flow $\dot{g}_{\bar{k} j}(t)=$ $-\left(R_{\bar{k} j}-g_{\bar{k} j}\right)$ so that

$$
\lambda \equiv \inf _{t \geq 0} \lambda_{\omega(t)}>0,
$$

where $\lambda_{\omega}$ is the lowest strictly positive eigenvalue of the Laplacian $\bar{\partial}^{\dagger} \bar{\partial}=$ $-g^{j \bar{k}} \nabla_{j} \nabla_{\bar{k}}$ acting on smooth $T^{1,0}(X)$ vector fields,

$$
\lambda_{\omega}=\inf _{V \perp H^{0}\left(X, T^{1,0}\right)} \frac{\|\bar{\partial} V\|^{2}}{\|V\|^{2}}
$$

Condition ( $\mathrm{S}$ ) can be interpreted as a stability condition in the following sense: Assume that there exist diffeomorphisms $F_{t}: X \rightarrow X$ so that $\left(F_{t}\right)^{*}(g(t))$ converges in $C^{\infty}$ to a metric $\tilde{g}(\infty)$. Then if $J$ is the complex structure of $X$, the pull-backs $\left(F_{t}\right)_{*}(J)$ converge also to a complex structure $J(\infty)$ (see $[\mathbf{1 1 2}], \S 4)$. Clearly, the eigenvalues $\lambda_{\omega(t)}$ are unchanged under $F_{t}$. If they do not remain bounded away from 0 as $t \rightarrow \infty$, then the complex structure $J(\infty)$ would have a strictly higher number of independent vector fields than $J$. Thus the $C^{\infty}$ closure of the orbit of $J$ under the diffeomorphism group contains a complex structure different from $J$, and $J$ cannot be included in a Hausdorff moduli space of complex structures.

Just as the condition (B) can be weakened to condition $\left(\mathrm{B}^{*}\right)$, we can strengthen condition $(S)$ to a condition $\left(\mathrm{S}^{*}\right)$ which requires more than we need, but which has now the advantage of not referring specifically to the Kähler-Ricci flow:

$\left(\mathrm{S}^{*}\right)$ Let $g_{m}$ be any sequence of metrics in $c_{1}(X)$ with $K$-energy bounded from above. Then the corresponding eigenvalues $\lambda_{g_{m}}$ are bounded uniformly from below by a strictly positive constant.

\section{The Necessity of Stability}

After describing at one end the analytic problem and at the other end the expected algebraic-geometric answer, we turn now to describing some of the partial results linking the two which have been obtained so far. The 
more complete results go in the direction of necessity, that is, the existence of constant scalar curvature metrics implies various forms of stability or semi-stability. We describe some of these results in this section.

\subsection{The Moser-Trudinger inequality and analytic $K$-stability} Consider first the case of $L=K_{X}^{-1}$ and thus Kähler-Einstein metrics. In this case, we have the following theorem of Tian [143], which shows the equivalence between the existence of a Kähler-Einstein metric and a properness property of the $F_{\omega_{0}}$ functional, and which led to the notion of $K$-stability (see Theorem 10 below):

THEOREM 8. Let $\left(X, \omega_{0}\right)$ be a compact manifold with $\left[\omega_{0}\right]=c_{1}(X)>0$. If $\operatorname{Aut}^{0}(X)=0$, then $X$ admits a Kähler-Einstein metric if and only if there exists $\gamma>0$ and constants $A_{\gamma}>0, B_{\gamma}$ so that

$$
F_{\omega_{0}}(\phi) \geq A_{\gamma} J_{\omega_{0}}(\phi)^{\gamma}-B_{\gamma}
$$

for all $\phi$ which are $\omega_{0}$-plurisubharmonic.

It was conjectured in $[\mathbf{1 4 3}]$ that the exponent $\gamma$ in the inequality $(7.1)$ can be taken to be $\gamma=1$. This conjecture, as well as the following extension to the case $\operatorname{Aut}^{0}(X) \neq 0$, was established in [104]:

TheOREM 9. Let $\left(X, \omega_{0}\right)$ be a compact manifold with $\left[\omega_{0}\right]=c_{1}(X)>0$. Let $G \subset \operatorname{Aut}^{0}(X)$ be any closed subgroup whose centralizer in the stabilizer $\operatorname{Stab}\left(\omega_{K E}\right)$ is finite. Then $X$ admits a $G$-invariant Kähler-Einstein metric if and only if there exists constants $A>0, B$, so that the following inequality holds for $G$-invariant $\omega_{0}$-plurisubharmonic potentials $\phi$,

$$
F_{\omega_{0}}(\phi) \geq A J_{\omega_{0}}(\phi)-B
$$

As a consequence of Theorem 8, Tian [143] deduced the following theorem, which implies in particular that the Iskovskih manifolds are smooth manifolds with $c_{1}(X)>0, \operatorname{Aut}^{0}(X)=0$ (and hence automatically vanishing Futaki invariant), and yet no Kähler-Einstein metrics. Define a special degeneration to be a one-parameter test configuration where the central fiber $X_{0}$ is a $\mathrm{Q}$-Fano variety. This means that $X_{0}$ is a normal variety, and if $K^{-1}$ is the anti-canonical line bundle on $X_{0}^{\text {reg }} \subseteq X_{0}$ (the smooth points of $X_{0}$ ) then there exists $L$, an ample line bundle on $X_{0}$ whose restriction to $X_{0}^{\text {reg }}$ is $K^{-1}$. In this set-up, by using a resolution of singularities, Ding and Tian [41] showed that one can define a generalized Futaki invariant $f_{X_{0}}$. This invariant can also be constructed explicitly as follows.

Let $\phi_{L^{k}}: X_{0} \rightarrow \mathbf{P}^{N}$ be a Kodaira imbedding corresponding to some basis $s_{j}$ of $H^{0}\left(X_{0}, L^{k}\right)$, let $h=\phi_{L^{k}}^{*} h_{F S}^{1 / k}$, which is a metric on the line bundle $K^{-1}$, and let $\omega=\frac{1}{k} \phi_{L^{k}}^{*} \omega_{F S}$, which is a Kähler metric on $X_{0}^{\text {reg }}$. Then $\omega^{n}$ defines a second metric on $K^{-1}$. Two metrics on the same holomorphic line bundle differ by a positive function: $\omega^{n}=h e^{-f / k}$. Thus $\operatorname{Ric}(\omega)-\omega=\frac{i}{2} \partial \bar{\partial} f$. In fact, we have an explicit formula for $f$ :

$$
f=-\log \sum\left|s_{j}\right|_{\omega}^{2}
$$


Let $V$ be the holomorphic vector field induced by the $\mathbf{C}^{\times}$action on $X_{0}^{\text {reg }}$. Then $f_{X_{0}}=\int_{M_{\text {reg }}} V(f) \omega^{n}$ is the generalized Futaki invariant defined previously by $[41]$.

TheOREM 10. [143] Let $X$ be a compact Kähler manifold with $c_{1}(X)>0$ and no holomorphic vector field. If $X$ admits a Kähler-Einstein metric, then $X$ is analytically $K$-stable in the sense that, for any non-trivial special degeneration, $f_{X_{0}}>0$.

A very rough sketch of the arguments in [143] is as follows. Let $X_{t} \subset$ $\mathbf{C P}^{N}$ be a special degeneration of a smooth projective variety $X$, and let $\phi_{t}$ be the Kähler potential of the Fubini-Study metric on $X_{t}$, pulled-back to $X$.

- If the degeneration is non-trivial, then $\sup _{t}\left\|\phi_{t}\right\|_{C^{0}}=\infty$.

- There exists constants $C_{1}, C_{2}$ so that $\left\|\phi_{t}\right\|_{C^{0}} \leq C_{1} J_{\omega_{0}}\left(\phi_{t}\right)+C_{2}$. This is a Harnack-type inequality, and follows from the uniformity of Sobolev constants for the family $X_{t}$ of subvarieties of $\mathbf{C P}^{N}$.

Assume next that $X$ has a Kähler-Einstein metric $\omega_{K E}$ and that Aut $^{0}$ $(X)=0$. By Theorem 8, it follows that $K_{\omega_{0}}\left(\phi_{t}\right) \rightarrow+\infty$ as $t \rightarrow 0$. On the other hand, we always have an expansion of the form

$$
K_{\omega_{0}}\left(\phi_{t}\right)=f_{X_{0}} \log \frac{1}{|t|}+O(1)
$$

as $t \rightarrow 0$ for a degeneration. It follows that $f_{X_{0}}>0$, as claimed. In the Iskovskih examples, $f_{X_{0}}=0$ always, and thus Theorem 10 implies that they do not admit Kähler-Einstein metrics.

7.2. Necessity of Chow-Mumford stability. Donaldson [45] has proved that the existence of a constant scalar curvature metric implies ChowMumford stability. More precisely, we have:

TheOREM 11. Let $X$ be a compact complex manifold and $L \rightarrow X$ a positive holomorphic line bundle. Assume that $X$ admits a metric $\omega \in c_{1}(L)$ of constant scalar curvature, and that $\operatorname{Aut}(X, L) / \mathbf{C}^{\times}$is discrete. Then $\left(X, L^{k}\right)$ is Hilbert-Mumford stable for $k$ sufficiently large.

The discreteness assumption was later weakened by Mabuchi [92].

The key intermediate notion for the proof of Theorem 11 is the notion of balanced imbedding. By the result of Zhang [166] (see also $\S 9.1$ below), the bundle $L \rightarrow X$ is $k$ Chow-Mumford stable if and only if $L^{k} \rightarrow X$ admits a balanced imbedding into $O(1) \rightarrow \mathbf{C P}^{N_{k}}$. Both notions of constant scalar curvature and of balanced imbedding are related to properties of the density of states $\rho_{k}(z)$. On one hand, by the Tian-Yau-Zelditch theorem and Lu's formula, we have the following expansion for the density of states $\rho_{k}(z)$,

$$
\rho_{k}(z)=k^{n}+\frac{1}{2} R(\omega) k^{n-1}+O\left(k^{n-2}\right)
$$

and thus, the condition that $R(\omega)$ is constant means that $\rho_{k}(z)$ is constant up to errors of order $O\left(k^{n-2}\right)$. On the other hand, if $h$ is a metric on 
$L$, with respect to which the density of states $\rho_{k}(z)$ is constant, then the orthonormal bases $\underline{s}=\left\{s_{\alpha}(z)\right\}_{\alpha=0}^{N_{k}}$ of $H^{0}\left(X, L^{k}\right)$ with respect to $h$ provide a balanced imbedding. Indeed, in view of the formula (4.8), we have then

$$
\omega-\frac{1}{k} \iota_{\underline{s}}^{*}\left(\omega_{F S}\right)=-\frac{i}{2 k} \partial \bar{\partial} \log \rho_{k}(z)=0,
$$

and hence

$$
\begin{aligned}
\frac{1}{V_{F S}} \int_{X} \frac{\overline{s_{\alpha}}(z) s_{\beta}(z)}{\sum_{\gamma=0}^{N_{k}}\left|s_{\gamma}(z)\right|^{2}} \iota_{\underline{s}}^{*}\left(\omega_{F S}\right)^{n} & =\frac{1}{V} \int_{X} \frac{\left\langle s_{\beta}, s_{\alpha}\right\rangle}{\rho_{k}} \omega^{n} \\
& =\frac{1}{\rho_{k} V} \int_{X}\left\langle s_{\beta}, s_{\alpha}\right\rangle \omega^{n}=\lambda \delta_{\beta \alpha}
\end{aligned}
$$

showing that the imbedding is balanced. Thus, heuristically, one would like to construct a metric $h$ with $\rho_{k}(z)$ constant. In practice, one proceeds in two steps. The first step uses the Tian-Yau-Zelditch theorem to produce metrics where $\rho_{k}(z)$ is constant to arbitrarily high order in $k^{-1}$, and whence a basis of $H^{0}\left(X, L^{k}\right)$ which is "approximately balanced". The second step shows how to construct a balanced metric from one which is approximately balanced.

In this section we give an outline of the two steps in the proof. The first is based directly on [45]. The second step is based on [45] together with the estimates proved in [108]. This step is motivated by the methods of [45] concerning moment maps and infinite dimesnsional symplectic geometry, but it does not make explicit use of these notions. Moreover, Deligne's formula for the curvature of the Deligne pairing, which plays a key role in the second step below, has an elementary proof (which is given in [107]) and one does not need to invoke Deligne's theory $[39]$ to implement the method (thus simplifying the presentation in $[\mathbf{1 0 8}])$.

7.2.1. Approximately balanced imbeddings. Donaldson [45] uses the TianYau-Zelditch theorem together with the formula of $\mathrm{Lu}$ [85] to prove the following two lemmas:

LEMMA 2. Assume that $\omega_{\infty} \in c_{1}(L)$ has constant scalar curvature and fix $q>0$. Then for $k>>0$ there exists a basis $\underline{s}=\underline{s}(k)$ of $H^{0}\left(X, L^{k}\right)$ with the following properties.

$$
M_{\bar{\alpha} \beta}=\frac{1}{V} \int_{\iota_{\underline{s}(k)}(X)} \frac{\bar{x}_{\alpha} x_{\beta}}{|x|^{2}} \omega_{F S}^{n}=\frac{k^{n}}{N+1} \delta_{\alpha \beta}+\left(E_{k}\right)_{\bar{\alpha} \beta}
$$

where $\iota_{\underline{s}}: X \rightarrow \mathbf{P}^{N_{k}}$ is the imbedding of $X$ via the basis $\underline{s}$ and, for $k$ sufficiently large,

$$
\left\|E_{k}\right\|_{o p} \leq k^{-q-1}
$$

where $\|\cdot\|_{o p}$ denotes the operator norm.

Remark: The matrix $E_{k}$ vanishes precisely when the basis $\underline{s}(k)$ is balanced. Thus we may think of $\underline{s}(k)$ as "approximately balanced". 
LEMma 3. There exists $C>0$ with the following properties. Let $\xi \in$ $\left.s u\left(N_{k}+1\right)\right)$, with $\|\xi\|_{\text {op }}=1$ and, for $t \in\left(-\frac{1}{10}, \frac{1}{10}\right)$ and $x \in \mathbf{P}^{N_{k}}$ with $k>>0$, let $\sigma_{t}(x)=\exp (i t \xi x)$. Let $\tilde{\omega}_{\infty}=k \omega_{\infty}$. Then

$$
\left\|\iota_{\underline{s}(k)}^{*} \sigma_{t}^{*} \omega_{F S}-\tilde{\omega}_{\infty}\right\|_{C^{4}\left(\tilde{\omega}_{0}\right)} \leq C t+O\left(\frac{1}{k}\right)
$$

Remark: Lemma 3 is a rewording of Proposition 27 in [45].

The idea of the proof of Lemma 2 goes as follows: Theorem 1 says that the density of states functions has an asymptotic expansion

$$
\rho_{k}(\omega)=k^{n}+A_{1}(\omega) k^{n-1}+A_{2}(\omega) k^{n-2}+\cdots
$$

where the $A_{p}$ are polynomials in the curvature tensor of $\omega$ and its covariant derivatives. If $\omega=\omega_{\infty}$ has constant scalar curvature, then Lu's theorem implies that $A_{1}\left(\omega_{\infty}\right)$ is constant. The key step in proving Lemma 2 is to construct a metric $\tilde{\omega}$ with the property

$$
\rho_{k}(\tilde{\omega})=k^{n}+\tilde{A}_{1} k^{n-1}+\tilde{A}_{2} k^{n-2}+\cdots
$$

where the $\tilde{A}_{j}$ are constant for $1 \leq j \leq q$. To do this, we let $\tilde{\omega}=\omega_{\infty}+\frac{i}{2 k} \partial \bar{\partial} \eta$ where $\eta$ is an unknown smooth function. Now the variation $\delta R$ of the scalar curvature under a variation $\delta \phi$ of the potential is given by

$$
\delta R=-\mathcal{D}^{*} \mathcal{D} \delta \phi+g^{j \bar{k}} \partial_{j} R \partial_{\bar{k}} \delta \phi,
$$

where $\mathcal{D}$ is the Lichnerowicz operator, mapping scalar functions to symmetric two-tensors

$$
\mathcal{D} \delta \phi=\nabla_{\bar{k}} \nabla_{\bar{j}} \delta \phi .
$$

Since $\omega$ has constant scalar curvature, we have then $A_{1}(\tilde{\omega})=A_{1}(\omega)+\frac{1}{k} \mathcal{L}(\eta)+$ $O\left(\frac{1}{k^{2}}\right)$ and $A_{2}(\tilde{\omega})=A_{2}(\omega)+O\left(\frac{1}{k}\right)$, where $\mathcal{L}(\eta) \equiv \mathcal{D}^{*} \mathcal{D}$. Under the assumption that $\operatorname{Aut}(L \rightarrow X)$ is discrete, $\mathcal{L}$ is a self-adjoint elliptic operator whose kernel consists only of the constants. Thus

$$
\rho_{k}(\tilde{\omega})=k^{n}+A_{1}\left(\omega_{\infty}\right) k^{n-1}+\left(A_{2}\left(\omega_{\infty}\right)+\mathcal{L}(\eta)\right) k^{n-2}+\cdots
$$

Now we choose $\tilde{A}_{2}$ to be the average of $A_{2}\left(\omega_{\infty}\right)$ and we choose $\eta$, the unique smooth function (up to additions of scalars) satisfying $\mathcal{L}(\eta)=\tilde{A}_{2}-A_{2}\left(\omega_{\infty}\right)$. This proves the result for $q=2$, and the statement for arbitrary $q$ is proved in a similar manner.

7.2.2. Estimates for the normal projection. This step consists of two lemmas which concern estimates for the normal projection operator $\pi_{N}$ under the assumption of $R$-bounded geometry. We first recall the definitions of " $R$-bounded geometry" (introduced in [45]) and of $\pi_{N}$ and then we state the results.

Definition of $R$-bounded geometry. Let $k$ be a positive integer, $R$ a positive real number and let $\tilde{\omega}_{0}=k \omega_{0}$. We say that a Kähler metric $\tilde{\omega} \in k c_{1}(L)$ is $R$-bounded if

$$
\tilde{\omega}>R^{-1} \tilde{\omega}_{0} \quad \text { and } \quad\left\|\tilde{\omega}-\tilde{\omega}_{0}\right\|_{C^{4}\left(\tilde{\omega}_{0}\right)}<R
$$


We say that a basis $\underline{s}$ of $H^{0}\left(X, L^{k}\right)$ is $R$-bounded if the corrseponding Bergman metric $\tilde{\omega}=\iota_{s}^{*} \omega_{F S}$ is $R$-bounded. Note that (7.10) implies that for $|t| \leq \rho$ and $k$ sufficiently large, the metric $\iota_{s}^{*} \sigma_{t}^{*} \omega_{F S}$ is $R$ bounded with $R=\frac{1}{2}$. In other words, the basis $\sigma_{t}(\underline{s})$ is $\frac{1}{2}$-bounded.

Definition of the operator $\pi_{N}$. Let $\underline{s}$ be a basis of $H^{0}\left(X, L^{k}\right)$ and consider the exact sequence of holomorphic vector bundles

$$
0 \rightarrow T X \rightarrow \iota_{\underline{s}}^{*} T \mathbf{P}^{N_{k}} \rightarrow Q \rightarrow 0
$$

where $Q \rightarrow X$ is, by defintion, the quotient bundle $\iota_{\underline{s}}^{*} T \mathbf{P}^{N_{k}} / T X$. Let $\mathcal{N} \subseteq$ $\iota_{\underline{s}}^{*} T \mathbf{P}^{N_{k}}$ be the orthogonal complement of $T X$ with respect to the FubiniStudy metric. Then we define $\pi_{T}: \iota_{s}^{*} T \mathbf{P}^{N_{k}} \rightarrow T X$ and $\pi_{N}: \iota_{s}^{*} T \mathbf{P}^{N_{k}} \rightarrow \mathcal{N}$ to be the corresponding projections. If $V \in \iota_{\underline{s}}^{*} T \mathbf{P}^{N_{k}}$ then we shall write $|V|_{F S}$ to be its norm with respect to the Fubini-Study metric on $T \mathbf{P}^{N_{k}}$ and we define

$$
\|V\|_{L^{2}\left(\iota_{\underline{s}}^{*} \omega_{F S}\right)}^{2}=\int_{X}|V|_{F S}^{2}\left(\iota_{\underline{s}}^{*} \omega_{F S}\right)^{n}
$$

With these notions in place, we can state the two lemmas. Fix $\xi \in$ $s u\left(N_{k}+1\right)$, with $\|\xi\|=1$. Let $X_{\xi}$ be the holomorphic vector field on $\mathbf{P}^{N_{k}}$ generated by $\xi$. More explicitly, let us recall that the holomorphic tangent bundle $T^{1,0}\left(\mathbf{P}^{N_{k}}\right)$ can be describe as follows.

$$
T^{1,0}\left(\mathbf{P}^{N_{k}}\right)=\left\{(z, v): z, v \in \mathbf{C}^{N_{k}+1}, z \neq 0\right\} / \sim
$$

where $(z, v) \sim\left(z^{\prime}, v^{\prime}\right)$ if and only if $z^{\prime}=\lambda v$ and $v^{\prime}=\lambda v+\mu z$ for some $\lambda, \mu \in \mathbf{C}$. Then $X_{\xi}: \mathbf{P}^{N_{k}} \rightarrow T^{1,0}\left(\mathbf{P}^{N_{k}}\right)$ is the $\operatorname{map} X_{\xi}(z)=(z, \xi z)$.

To simplify the exposition, we assume throughout that Aut $(X)$ is discrete (this assumption can be removed - see [108] for details).

LEMMA 4. Let $\underline{s}$ be a basis of $H^{0}\left(X, L^{k}\right)$ with $\frac{1}{2}$-bounded geometry. Then there exists $C_{1}>1$ such that

$$
1 \leq C_{1} k\left\|X_{\xi}\right\|_{L^{2}\left(\iota_{\underline{s}}^{*} \omega_{F S}\right)}^{2}
$$

LEMmA 5. Let $\underline{s}$ be a basis of $H^{0}\left(X, L^{k}\right)$ with $\frac{1}{2}$-bounded geometry. Then there exists $C_{2}>1$ such that

$$
\left\|\pi_{T} X_{\xi}\right\|_{L^{2}\left(\iota_{\underline{s}}^{*} \omega_{F S}\right)}^{2} \leq C_{2} k\left\|\pi_{N} X_{\xi}\right\|_{L^{2}\left(\iota_{\underline{s}}^{*} \omega_{F S}\right)}^{2}
$$

Remark: The assumption that Aut $(X)$ is discrete implies that $X_{\xi}$ is not tangent to $\iota_{\underline{s}}(X)$ and thus, $\left\|\pi_{N} X_{\xi}\right\|_{L^{2}\left(\iota_{\underline{s}}^{*} \omega_{F S}\right)}^{2}>0$. Lemma 5 shows that $\left\|\pi_{N} X_{\xi}\right\|_{L^{2}\left(\iota_{\underline{s}}^{*} \omega_{F S}\right)}^{2}$ cannot be too close to zero.

7.2.3. Synthesis. We show how steps one and two can be combined to prove Theorem 11. We must show that the existence of the almost balanced basis $\underline{s}(k)$ implies the existence of a balanced basis. For this, it is simplest to reformulate the problem in terms of the functional $-F_{\iota_{s}^{*}\left(\omega_{F S}\right)}^{0}$ : our task is to find a critical point (in fact, a minimum) for this functional. This is now 
easy, since section $\S 7.2 .1$ provides upper bounds for the absolute value of its first derivatives, while section $\S 7.2 .2$ provides a positive lower bound for its second derivative.

We first introduce some necessary notation: Let $\tilde{\omega}(k)=\iota_{\underline{s}(k)}^{*} \omega_{F S}$. If $\sigma \in S L\left(N_{k}+1, \mathbf{C}\right)$ we let $\phi_{\sigma}(x)=\log \frac{|\sigma(x)|^{2}}{|x|^{2}}$. Then to prove existence of a balanced metric, in view of Theorem 3 , it suffices to show that $-F_{\tilde{\omega}(k)}^{0}\left(\phi_{\sigma}\right)$ achieves its minimum at some point $\sigma \in S L\left(N_{k}+1\right)$. Fix $\xi \in s u\left(N_{k}+1\right)$ and let $\sigma_{t}(x)=\exp (i t \xi x)$ and $\phi_{t}(x)=\phi_{\sigma(t)}$. Consider

$$
-(n+1) V_{F S} F^{0}(t)=-(n+1) V_{F S} F_{\tilde{\omega}(k)}^{0}\left(\phi_{t}\right)=\int_{\iota_{\underline{s}}(X)} \phi_{t} \cdot \sum_{j=0}^{n}\left(\sigma_{t}^{*} \omega_{F S}^{j} \wedge \omega_{F S}^{n-j}\right)
$$

Then (5.7) combined with Lemma 2 yields

$$
\begin{aligned}
\left|(n+1) \dot{F}^{0}(0)\right| & =\left|\frac{1}{V} \int_{\iota_{\underline{s}}(X)} \frac{x^{*} \xi x}{x^{*} x} \cdot \omega_{\mathrm{FS}}^{n}\right|=\left|\operatorname{Tr}\left(\xi E_{k}\right)\right| \\
& \leq\left(N_{k}+1\right)^{\frac{1}{2}}\|\xi\| \cdot\left\|E_{k}\right\|_{\mathrm{op}} \leq k^{n / 2-q-1}
\end{aligned}
$$

On the other hand we have the following formula of Deligne [39] for $\ddot{F}^{0}$ (see (5.7) and [107] for an elementary proof):

$$
-\ddot{F}^{0}(t)=\left\|\pi_{N} X_{\xi}\right\|_{L^{2}\left(\iota_{\underline{s}}^{*} \sigma_{t}^{*} \omega_{F S}\right)}^{2}=\int_{X}\left|\pi_{N} X_{\xi}\right|_{F S}^{2} \cdot\left(\iota_{\underline{s}}^{*} \sigma_{t}^{*} \omega_{F S}\right)^{n}
$$

To estimate $\left\|\pi_{N} X_{\xi}\right\|^{2}$ we wish to use Lemma 4 and Lemma 5 . First we apply Lemma 3 to deduce that the basis $\sigma_{t} \cdot \underline{s}(k)$ is $\frac{1}{2}$-bounded for $|t| \leq \frac{1}{4 C}$ and for $k \gg 0$. Now Lemma 4 and Lemma 5 imply that for such $t$,

$$
\begin{aligned}
-\ddot{F}^{0}(t) & \geq \frac{1}{C_{2}(k+1)}\left(C_{2} k\left\|\pi_{N} X_{\xi}\right\|^{2}+\left\|\pi_{N} X_{\xi}\right\|^{2}\right) \\
& \geq \frac{1}{C_{2}(k+1)}\left\|X_{\xi}\right\|^{2} \geq \frac{1}{C_{1} C_{2} k(k+1)}
\end{aligned}
$$

Thus, if we take $q>n / 2+1$, comparing (7.22) and (7.24) we see that, for $k$ sufficiently large,

$$
-F^{0}(t)>-F^{0}(0) \quad \text { if } \quad|t| \geq \frac{1}{4 C_{1}} .
$$

This shows that $-F_{\tilde{\omega}(k)}^{0}\left(\phi_{\sigma}\right)$ achieves its minimum at some point $\sigma \in S L$ $\left(N_{k}+1\right)$ which is of the form $\sigma=\exp (i \xi)$ where $\xi \in s u\left(N_{k}+1\right)$ and $\|\xi\| \leq \frac{1}{4 C_{1}}$.

7.3. Necessity of semi $\boldsymbol{K}$-stability. The following theorem due to Donaldson [50] provides an attractive lower bound for $\|R(\omega)-\mu n\|_{L^{2}}^{2}$ in terms of Futaki invariants of test degenerations: 
TheOREM 12. Let $L \rightarrow X$ be a positive line bundle. Then we have

$$
\inf _{\omega \in c_{1}(L)}\|R(\omega)-\mu n\|_{L^{2}} \geq \sup _{\mathcal{T}}\left(-\frac{\mathrm{F}(\mathcal{T})}{D(\mathcal{T})}\right)
$$

where the supremum on the right hand side runs over all test configurations $\mathcal{T}$, and the invariant $D(\mathcal{T})$ of a test configuration $\mathcal{T}$ is defined by,

$$
D(\mathcal{T})=\lim _{k \rightarrow+\infty} \frac{\left\|A_{k}\right\|}{k^{\frac{n}{2}+1}} .
$$

where $\|A\|=\left(\sum_{i, j}\left|a_{i j}\right|^{2}\right)^{1 / 2}$ is the Hilbert-Schmidt norm.

This implies immediately the following corollary:

Corollary 1. If $L \rightarrow X$ is a positive line bundle over a compact complex manifold, and if $X$ admits a metric of constant scalar curvature in $c_{1}(L)$, then $L \rightarrow X$ is $K$-semistable.

Another lower bound for the Calabi energy involving instead a version of the Futaki invariant along geodesic rays can be found in [28].

In the particular case when $L=K_{X}^{-1}$, it is known that the boundedness of the Mabuchi $K$-energy from below on $c_{1}(L)$ implies that inf ${ }_{\omega \in c_{1}(L)} \| R(\omega)-$ $\mu n \|_{L^{2}}=0$ (see e.g. $[\mathbf{7}, \mathbf{1 1 1}, \mathbf{1 0 5}]$ ). Thus we also have:

Corollary 2. Let $X$ be a compact complex manifold with $c_{1}(X)>$ 0 . If the Mabuchi $K$-energy is bounded from below on $c_{1}(X)$, then $X$ is K-semistable.

Lower bounds for the $K$-energy have been obtained in certain cases by Weinkove [155] using the $J$-flow.

The proof of Theorem 12 is so basic and elegant that we can include it here. In fact, it relies on the properties of $F_{\omega_{0}}^{0}(\phi)$ on the spaces $\mathcal{K}_{k}$ of Bergman metrics which have already been discussed in section $\S 5$. The version that we present here is slightly shorter, thanks to the use of Lemma 6 below.

Let $\mathcal{T}$ be a test configuration, and let $A_{k}, B_{k}$ be the endomorphisms on $H^{0}\left(X_{0}, L_{0}^{k}\right)$ as defined in section $\S 6$ 1.6. Let $\sigma_{t}^{(k)}=e^{t B_{k}}: \mathbf{R} \rightarrow G L\left(N_{k}+1\right)$, $\underline{\sigma}_{t}^{(k)}=e^{t A_{k}}: \mathbf{R} \rightarrow S L\left(N_{k}+1\right)$ be the one-parameter subgroup defined by $B_{k}$ and $A_{k}$. For $k>>0$ embed $X \subseteq \mathbf{C P}^{N_{k}}$ by a basis $\underline{s}$ of $H^{0}\left(X, L^{k}\right)$. Let $\phi_{t}(x)=\log \frac{\left|\sigma_{t}^{(k))}(x)\right|^{2}}{|x|^{2}}$ and define

$$
\mu\left(A_{k}\right)=-\lim _{t \rightarrow-\infty} V_{F S} \dot{F}_{\iota_{\underline{s}}^{*}\left(\omega_{F S}\right)}^{0}\left(\phi_{t}\right)
$$

Then we have the following alternative description of the Donaldson-Futaki invariant of the test configuration $\mathcal{T}[\mathbf{1 1 4}]$.

Lemma 6. Let $\mathcal{T}$ be a test configuration. Then

$$
F(\mathcal{T})=\lim _{k \rightarrow \infty} \frac{\mu\left(A_{k}\right)}{k^{n}}
$$


Assuming the lemma for the moment, we can give the proof of the theorem. Fix $h$ a metric on $L$, and let $\underline{s}$ be the Kodaira imbedding defined by an orthonormal basis $\underline{s}$ of $H^{0}\left(X, L^{k}\right)$ with respect to the $L^{2}$ norm defined by $h$ and $\omega=-\frac{i}{2} \partial \bar{\partial} \log h$. By the convexity of $F_{\iota_{\underline{s}}^{*}\left(\omega_{F S}\right)}^{0}$ along one-parameter subgroups, we have

$$
-\lim _{t \rightarrow-\infty} V_{F S} \dot{F}_{\iota_{\underline{s}}^{*}\left(\omega_{F S}\right)}^{0}(t) \leq-V_{F S} \dot{F}_{\iota_{\underline{s}}^{*}\left(\omega_{F S}\right)}^{0}(0)
$$

We can now evaluate both sides of the equation, using Lemma 6 for the numerical invariant $\mu\left(A_{k}\right)$ and $(5.7)$ for $-\dot{F}_{\iota_{\underline{s}}^{*}\left(\omega_{F S}\right)}\left(\right.$ with $\delta \sigma=\delta \sigma^{*}=A_{k}$ ). This gives

$$
\frac{\mu\left(A_{k}\right)}{\left\|A_{k}\right\|} \leq V_{F S} \frac{\operatorname{Tr}\left(A_{k} \cdot \underline{M}\right)}{\left\|A_{k}\right\|} \leq V_{F S}\|\underline{M}\|,
$$

where $\underline{M}$ is the traceless part of the matrix $M$ defined by (5.8) and (5.15), and thus given by

$$
\underline{M}_{\alpha \bar{\beta}}=-\frac{1}{k V_{F S}} \int_{X}\left\langle s_{\alpha}, s_{\beta}\right\rangle(R-\bar{R}) \omega^{n}+O\left(\frac{1}{k^{n+2}}\right) .
$$

We estimate the Hilbert-Schmidt norm of $\underline{M}$. By an orthonormal transformation, we may assume that $\underline{M}_{\alpha \bar{\beta}}$ is diagonal. The diagonal entries can be estimated by the Cauchy-Schwarz inequality and the fact that $\left\|s_{\alpha}\right\|_{L^{2}}^{2}=1$,

$$
\begin{aligned}
V_{F S} \underline{M}_{\alpha \bar{\alpha}} & =-\frac{1}{k} \int_{X}|s|_{h^{k}}^{2}(R(\omega)-\bar{R}) \omega^{n}+O\left(\frac{1}{k^{2}}\right) \\
& \leq \frac{1}{k}\left(\int_{X}|s|_{h^{k}}^{2}|R(\omega)-\bar{R}|^{2} \omega^{n}\right)^{\frac{1}{2}}+O\left(\frac{1}{k^{2}}\right) .
\end{aligned}
$$

Applying the Tian-Yau-Zelditch theorem, we obtain

$$
\begin{aligned}
V_{F S}^{2} \sum_{\alpha=0}^{N_{k}} \underline{M}_{\alpha \bar{\alpha}}^{2} & \leq \frac{1}{k^{2}} \int_{X} \rho_{k}(z)|R(\omega)-\bar{R}|^{2} \omega^{n}+O\left(\frac{1}{k^{-n+3}}\right) \\
& \leq \frac{1}{k^{-n+2}}\|R(\omega)-\bar{R}\|_{L^{2}}^{2}+O\left(\frac{1}{k^{-n+3}}\right) .
\end{aligned}
$$

Substituting this bound into (7.31) and letting $k \rightarrow \infty$ gives the desired statement. Q.E.D.

Proof of Lemma 6: We begin by recalling some basic facts about the Mumford numerical invariant for projective varieties. Let $Z \subseteq \mathbf{P}^{N}$ be a smooth subvariety, and $B$ an $(N+1) \times(N+1)$ matrix. Let $\omega_{F S}$ be the Fubini-Study metric on $\mathbf{P}^{N}$. We shall also denote by $\omega_{F S}$ the restriction of the FubiniStudy metric to $Z$. For $t \in \mathbf{R}$ let $\sigma_{t} \in G L(N+1, \mathbf{C})$ be the matrix $\sigma_{t}=e^{t B}$ and let $\psi_{t}: \mathbf{P}^{N} \rightarrow \mathbf{R}$ be the function

$$
\psi_{t}(z)=\log \frac{\left|\sigma_{t} z\right|^{2}}{|z|^{2}}
$$


Here we view $z$ as an element in $\mathbf{P}^{N}$ and, when there is no fear of confusion, a column vector in $\mathbf{C}^{N+1}$.

Then $\psi_{t}$ is a smooth path in $\mathcal{H}$ : In fact, $\sigma_{t}^{*} \omega_{F S}=\omega_{F S}+\frac{i}{2} \partial \bar{\partial} \psi_{t}$. Define

$$
\mu(Z, B)=-\lim _{t \rightarrow-\infty} V_{F S} \dot{F}_{\omega_{F S}}^{0}\left(\psi_{t}\right)=-\operatorname{dot} V_{F S} F^{0}(-\infty)
$$

where $V_{F S}$ denotes the volume of $Z$ with respect to $\omega_{F S}$. The function $F^{0}(t)=F_{\omega_{F S}}^{0}\left(\psi_{t}\right): \mathbf{R} \rightarrow \mathbf{R}$ is convex so the above limit exists. Suppose $\lambda: \mathbf{C}^{\times} \rightarrow G L(N+1, \mathbf{C})$ is an algebraic homomorphism, let $B \in g l(N+1, \mathbf{C})$ be such that $\lambda\left(e^{t}\right)=e^{t B}$ for all $t \in \mathbf{R}$ and $Z^{(0)}=\lim _{\tau \rightarrow 0} \lambda(\tau)(Z)$ the flat limit of $Z$. Thus $Z^{(0)} \subseteq \mathbf{P}^{N}$ is a subscheme of $\mathbf{P}^{N}$ with the same Hilbert polynomial as $Z$. Let $M_{0}=\left.O(1)\right|_{Z^{(0)}}$ and $M=\left.O(1)\right|_{Z}$. We assume that $Z \subseteq \mathbf{P}^{N}$ is an imbedding of $Z$ by a basis of $H^{0}(Z, M)$. Then $\lambda(\tau)$ defines an automorphism of $H^{0}\left(Z^{(0)}, M_{0}^{p}\right)$ and, for $p>0$, we let $\tilde{w}(Z, B, p)$ be the weight of this action on $\operatorname{det}\left(H^{0}\left(Z_{0}, M_{0}^{p}\right)\right)$. One easily sees that

$$
\tilde{w}(Z, B, 1)=\operatorname{Tr}(B)
$$

and, if $I$ is the identity matrix,

$$
\tilde{w}(Z, I, p)=p \operatorname{dim} H^{0}\left(Z^{(0)}, M^{p}\right)
$$

It is then known by the work of Mumford [97] and Zhang [166] that $\tilde{w}(Z, B, p)$ is a polynomial in $p$ for $p$ large such that for $B=A \in \operatorname{sl}(N+1, \mathbf{C})$ we have

$$
\tilde{w}(Z, A, p)=\frac{\mu(Z, A)}{(n+1) !} \cdot p^{n+1}+O\left(p^{n}\right) \text { and } \tilde{w}\left(Z^{(0)}, A, 1\right)=0
$$

We apply these general remarks to the case considered in Lemma 6 , that is, to the case $B=B_{k}$. Thus we let $L \rightarrow X$ be an ample line bundle, and $\rho: \mathbf{C}^{\times} \rightarrow \operatorname{Aut}(\mathcal{L} \rightarrow \mathcal{X} \rightarrow \mathbf{C})$ a test configuration $\mathcal{T}$. Let $k$ be an integer such that $L^{k}$ is very ample. By Lemma 1 , there exists a basis $\underline{s}$ of $H^{0}\left(X, L^{k}\right)$, and an imbedding

$$
I_{\underline{s}}:\left(\mathcal{L}^{k} \rightarrow \mathcal{X} \rightarrow \mathbf{C}\right) \rightarrow\left(O(1) \times \mathbf{C} \rightarrow \mathbf{C P}^{N_{k}} \times \mathbf{C} \rightarrow \mathbf{C}\right)
$$

which restricts to $\iota_{\underline{s}}$ on the fiber $X_{1}$ and intertwines $\rho(\tau)$ and $B_{k}$, i.e.,

$$
I_{\underline{s}}\left(\rho(\tau) l_{w}\right)=\left(\tau^{B_{k}} \cdot I_{\underline{s}}\left(l_{w}\right), \tau w\right) \quad \text { for each } l \in \mathcal{L}^{k}
$$

Let $Z_{k} \subseteq \mathbf{C P}^{N_{k}}$ be the image of $X$ by the map $\iota_{\underline{s}}$. Then, $\left(L^{r}\right)^{p}=L^{r p}$ implies (with $k=r p$ )

$$
\tilde{w}\left(Z_{r}, B_{r}, p\right)=\tilde{w}\left(Z_{k}, B_{k}, 1\right)=\operatorname{Tr}\left(B_{k}\right)
$$

In particular we see that $\operatorname{Tr}\left(B_{k}\right)$ is a polynomial in $k$ of degree $n+1$ (for $k>>1$ ).

On the other hand, $A_{k}=B_{k}-\frac{1}{d_{k}} \operatorname{Tr}\left(B_{k}\right)$ (where $d_{k}=N_{k}+1$ ) implies

$$
\begin{aligned}
r d_{r} \operatorname{Tr}\left(B_{k}\right) & =r d_{r} \tilde{w}\left(Z_{r}, B_{r}, p\right)=\tilde{w}\left(Z_{r}, r d_{r} B_{r}, p\right) \\
& =\tilde{w}\left(Z_{r}, r d_{r} A_{r}, p\right)+\tilde{w}\left(Z_{r}, r \operatorname{Tr}\left(B_{r}\right), p\right)
\end{aligned}
$$


Thus

$r d_{r} \operatorname{Tr}\left(B_{k}\right)-k d_{k} \operatorname{Tr}\left(B_{r}\right)=r d_{r} \tilde{w}\left(Z_{r}, A_{r}, p\right)=e_{T}(r) k^{n+1}+O\left(k^{n}\right)$ for $k \gg 1$

where $e_{T}$ is a polynomial in $r$ (for $r \gg 1$ ) of degree at most $n$. If follows from the definition of $F(T)$ that $F(T)$ is the leading coefficient of $e_{T}(r)$. Comparing with (7.39) we get

$$
\lim _{r \rightarrow \infty} \frac{\mu\left(Z_{r}, r N_{r} A_{r}\right)}{r^{n} r^{n+1}(n+1) !}=F(T)
$$

Since $r^{-n} N_{r}=\frac{1}{n !} \int \omega^{n}+O\left(r^{-1}\right)$, we obtain the desired formula.

\section{Sufficient Conditions: the Kähler-Einstein Case}

We describe next some of what is known in the direction of sufficiency of stability conditions. The case of Kähler-Einstein is better understood, so we begin with this case. As noted earlier, the constant scalar curvature equation reduces to a complex Monge-Ampère equation, which can be investigated either as an elliptic equation by the method of continuity, or as a parabolic flow, giving rise to the Kähler-Ricci flow.

8.1. The $\alpha$-invariant. An approach to the problem of Kähler-Einstein metrics is through the $\alpha$-invariant, defined on a compact Kähler manifold $\left(X, \omega_{0}\right)$ as follows $[\mathbf{1 3 9}]$,

$$
\alpha(X)=\sup \left\{\kappa>0 ; \sup _{\phi} \int_{X} e^{-\kappa \phi} \omega_{0}^{n}<\infty\right\},
$$

where the supremum on the right hand side is taken over all $\phi$ which are $\omega_{0}$ plurisubharmonic and normalized by $\sup _{X} \phi=0$. It was shown in Tian [139], using Hörmander's estimate for subharmonic functions [73], that $\alpha(X)>0$, and that the lower bound $\alpha(X)>\frac{n}{n+1}$ would imply the existence of a Kähler-Einstein metric on $X$. Other applications of lower bounds for $\alpha(X)$ are in [146]. For toric manifolds, the $\alpha$-invariant has been completely determined in Song [128], generalizing earlier special cases established in Batyrev and Selivanova $[\mathbf{9}]$ and $[\mathbf{1 2 7}]$. It has very recently been evaluated for the Mukai-Umemura 3-fold in Donaldson [53]. More general exponential estimates for plurisubharmonic functions with respect to certain probability measures are in Dinh, Nguyen, and Sibony [42]. The important role of an $\alpha$-invariant for tame symplectic manifolds, if it is strictly positive, has been brought to light by Tosatti, Weinkove, and Yau [151].

8.2. Nadel's multiplier ideal sheaves criterion. In the case of vector bundles, as proved by Donaldson [43] and Uhlenbeck-Yau [152], the obstruction to the existence of a Hermitian-Einstein metric is precisely the presence of destabilizing subsheaves in the sense of Mumford-Takemoto. It is natural to expect that the obstruction to Kähler-Einstein metrics, and more generally, to Kähler metrics of constant scalar curvature, can ultimately be 
also encoded in suitable notions of destabilizing sheaves. An early important result is the following theorem of Nadel [98], which we quote here in the simpler version of Demailly-Kollár [40]. Let $\left(X, \omega_{0}\right)$ be a compact Kähler manifold with $c_{1}(X)>0$. For each $\omega_{0}$-plurisubharmonic $\psi$ (that is, upper semi-continuous and satisfying $\omega_{0}+\frac{i}{2} \partial \bar{\partial} \psi \geq 0$ ), define the multiplier ideal sheaf $\mathcal{I}(\psi)$ by

$$
\mathcal{I}_{z}(\psi)=\left\{f ; \exists U \ni z, f \in \mathcal{O}(U), \int_{X} e^{-\psi}|f|^{2} \omega_{0}^{n}<\infty\right\}
$$

THEOREM 13. If $\left(X, \omega_{0}\right)$ does not admit a Kähler-Einstein metric, then for any $p \in\left(\frac{n}{n+1}, 1\right]$, there exists a $\omega_{0}$-plurisubharmonic function $\psi$ so that the multiplier ideal sheaf $\mathcal{I}(p \psi)$ defines a proper, coherent analytic sheaf on $X$ with acyclic cohomology, i.e.,

$$
H^{q}(X, \mathcal{J}(p \psi))=0, \quad q \geq 1 .
$$

If $X$ admits a compact subgroup $G$ of holomorphic automorphisms, and $\omega_{0}$ is $G$-invariant, then $\mathcal{I}(p \psi)$ and the corresponding subscheme are also $G$ invariant.

Multiplier ideal sheaves were introduced by Kohn $[\mathbf{7 8}]$ in the context of subelliptic estimates for the $\bar{\partial}$-Neumann problem. Their applications to complex geometry have been pioneered by Siu $[\mathbf{1 2 4}, \mathbf{1 2 5}]$.

The original proof of Nadel's theorem [98] is based on the method of continuity for the complex Monge-Ampère equation (3.5),

$$
\left(\omega_{0}+\frac{i}{2} \partial \bar{\partial} \phi\right)^{n}=e^{f_{0}-t \phi} \omega_{0}^{n}, \quad 0 \leq t \leq 1 .
$$

By Yau's estimates [157], a $C^{\infty}$ solution of (3.5) exists if the equation (8.4) admits a $C^{0}$ a priori estimate

$$
\sup _{t}\|\phi\|_{C^{0}} \leq C<\infty .
$$

On the other hand, the solutions of the equation (8.4) satisfy the following $[123,139]$

$$
\begin{aligned}
\frac{1}{V} \int_{X}(-\phi) \omega_{\phi}^{n} & \leq n \frac{1}{V} \int_{X} \phi \omega_{0}^{n} \\
\operatorname{osc} \phi & \leq A \frac{1}{V} \int_{X} \phi \omega_{0}^{n}+B
\end{aligned}
$$

The second inequality is a Harnack-type inequality which can be proved by Moser iteration, since the equation (8.4) implies that the Ricci curvature of $\omega_{\phi}$ is bounded from below for $t \geq \epsilon>0$, and the Sobolev constants with respect to $\omega_{\phi}$ are then uniformly bounded from below. Another important observation is the following lemma:

LEMMA 7. If there exists a constant $p \in\left(\frac{n}{n+1}, 1\right]$ so that

$$
\sup _{t} \frac{1}{V} \int_{X} \exp \left(-p\left(\phi-\frac{1}{V} \int_{X} \phi \omega_{0}^{n}\right)\right) \omega_{0}^{n}<\infty,
$$


then it follows that

$$
\sup _{t} \frac{1}{V} \int_{X} \phi \omega_{0}^{n} \leq C<\infty .
$$

Proof. Since the logarithm is concave, the given inequality implies

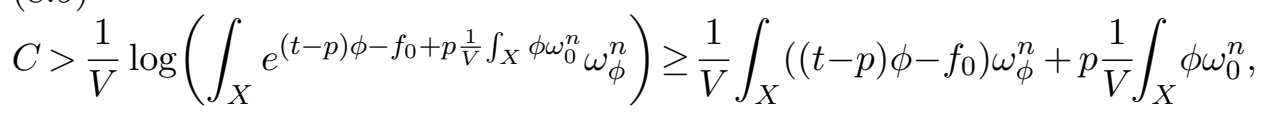

and thus, in view of (8.6),

$$
p \frac{1}{V} \int_{X} \phi \omega_{0}^{n} \leq(t-p) \frac{1}{V} \int_{X}(-\phi) \omega_{\phi}^{n}+C \leq n(1-p) \frac{1}{V} \int_{X} \phi \omega_{0}^{n}+C .
$$

where the last inequality applies if $\frac{1}{V} \int_{X}(-\phi) \omega_{\phi}^{n} \geq 0$. The desired bound follows in this case if $p>n(1-p)$. When $\frac{1}{V} \int_{X}(-\phi) \omega_{\phi}^{n}<0$, we just observe that it suffices to prove the upper bound on $\frac{1}{V} \int_{X} \phi \omega_{0}^{n}$ for $t \geq t_{0}$, for some fixed small time $t_{0}>0$. Since the given bound (8.7) implies a similar bound for $p_{0}=\frac{1}{2} t_{0}$, we can just apply the first inequality in (8.8) with $p \rightarrow p_{0}$ and deduce that $\frac{1}{V} \int_{X} \phi \omega_{0}^{n}$ is uniformly bounded from above. Q.E.D.

The proof of Nadel's theorem can now be completed as follows. If $X$ does not admit a Kähler-Einstein metric, then the $C^{0}$ estimate (8.5) must fail. Now, if we normalize $f_{0}$ by

$$
\frac{1}{V} \int_{X} e^{f_{0}} \omega_{0}^{n}=1
$$

then the equation (8.4) implies that $\phi$ must vanish somewhere, and thus $\|\phi\|_{C^{0}} \leq \operatorname{osc} \phi$. It follows that $\operatorname{osc} \phi$ is unbounded, and in view of the Harnack inequality (8.6) that $\frac{1}{V} \int_{X} \phi \omega_{0}^{n}$ is unbounded. By Lemma 7, for any $p \in\left(\frac{n}{n+1}, 1\right]$, the left hand side of the inequality (8.7) is unbounded for some sequence $\phi=\phi\left(t_{j}\right)$. Let $\psi$ be a limit point of $\phi\left(t_{i}\right)-\frac{1}{V} \int_{X} \phi\left(t_{i}\right) \omega_{0}^{n}$. By the semi-continuity theorem of Demailly-Kollár [40], $e^{-p \psi}$ is not in $L^{1}$, and thus $\mathcal{I}(p \psi)$ is a proper sheaf. It is also coherent and acyclic as a consequence of the general theory of multiplier ideal sheaves. This completes the proof of Theorem 13.

8.3. The Kähler-Ricci flow. Although there has been important developments since the late 1980's, particularly concerning complex surfaces $[\mathbf{1 4 6}, \mathbf{1 4 1}]$, we jump now to the more recent progresses, based on the Kähler-Ricci flow.

Let $\left(X, \omega_{0}\right)$ be a compact Kähler manifold, with $\mu \omega_{0} \in c_{1}(X)$. Recall that the Kähler-Ricci flow is the flow of metrics defined by (3.8). Since it preserves the Kähler class, we may write as usual $g_{\bar{k} j}=\left(g_{0}\right)_{\bar{k} j}+\partial_{j} \partial_{\bar{k}} \phi$, $\omega_{\phi}=\omega_{0}+\frac{i}{2} \partial \bar{\partial} \phi$, and the flow for the metrics $g_{\bar{k} j}$ is equivalent to the flow (3.9) for the potentials $\phi$. It follows readily from the maximum principle 
that

$$
\|\phi\|_{C^{M}(X \times[0, T))} \leq C_{M T}
$$

and thus the flow exists for all $t \in[0, \infty)[\mathbf{2 2}]$. The main issue is the convergence of the flow. It has been shown to converge when $\mu<0$ and $\mu=0[\mathbf{2 2}]$, and thus we restrict to the case $\mu>0$, which is the case of positive curvature. Clearly $\mu$ can be normalized to be $\mu=1$, and we shall do so henceforth. For the convenience of the reader, we reproduce here the equation

$$
\dot{\phi}=\log \frac{\omega_{\phi}^{n}}{\omega_{0}^{n}}+\phi-f_{0}, \quad \phi(0)=c_{0}
$$

where $c_{0}$ is a constant, and $f_{0}$ is the Ricci potential of the initial Kähler form, defined with normalization by

$$
\operatorname{Ric}\left(\omega_{0}\right)-\omega_{0}=\frac{i}{2} \partial \bar{\partial} f_{0}, \quad \frac{1}{V} \int_{X} e^{f_{0}} \omega_{0}^{n}=1 .
$$

8.3.1. Perelman's estimates. The following are key estimates for the Kähler-Ricci flow. They are all consequences of Perelman's crucial monotonicity formulas $[\mathbf{1 0 1}, \mathbf{3 6}, \mathbf{1 2 9}]$, with the first three statements directly due to him, and the last one to Ye $[\mathbf{1 6 1}]$ and Zhang [165]:

THEOREM 14. (i) The Ricci potential $f$ satisfies the following estimates along the Kähler-Ricci flow,

$$
\sup _{t \geq 0}\left(\|f\|_{C^{0}}+\|\nabla f\|_{C^{0}}+\|\Delta f\|_{C^{0}}\right)<\infty .
$$

(ii) The diameters of $X$ with respect to the metrics $g_{\bar{k} j}(t)$ are uniformly bounded for $t \geq 0$.

(iii) Let $\rho>0$ be fixed. Then there exists a constant $c>0$ so that for all $x \in X, t \geq 0$, and $r$ with $0<r \leq \rho$, we have

$$
\int_{B_{r}(x)} \omega_{\phi}^{n}>c r^{2 n}
$$

where $B_{r}(x)$ is the geodesic ball centered at $x$ of radius $r$ with respect to the metric $g_{\bar{k} j}(t)$.

(iv) There exists a constant $C$, independent of $t \geq 0$, so that the Sobolev inequality

$$
\|u\|_{L^{\frac{2 n}{n-1}}} \leq C\left(\|\nabla u\|_{L^{2}}+\|u\|_{L^{2}}\right), \quad u \in C^{\infty}(X),
$$

holds, with all norms taken with respect to the metric $g_{\bar{k} j}(t)$.

All these estimates are for quantities which depend only on the metrics $g_{\bar{k} j}$, and not on the potentials $\phi$ and the normalization $c_{0}$ for the initial potential. To translate them into estimates for $\phi$, we need to pick a precise normalization for $\phi$. First, we note that the quantity $\int_{0}^{\infty}\|\nabla \dot{\phi}\|_{L^{2}}^{2} e^{-t} d t$ (first written down in $[\mathbf{3 4}]$; see also [84]) is finite and independent of the choice of initial condition $c_{0}$. This is because two solutions of (8.13) with different 
initial values $c_{0}$ and $\tilde{c}_{0}$ differ by the expression $\left(\tilde{c}_{0}-c_{0}\right) e^{t}$, which cancels out in the norm $\|\nabla \dot{\phi}\|_{L^{2}}^{2} \equiv\|\nabla \dot{\phi}\|^{2}$. Furthermore,

$$
\dot{\phi}=-f+\alpha(t)
$$

for some $\alpha(t)$ independent of $z$, and by Perelman's estimate (i) in Theorem 14 above, the quantity $\|\nabla \dot{\phi}\|^{2}$ is bounded, and hence the integral in $t$ converges. It is then shown in $[\mathbf{1 0 3}]$ that, if the initial value $c_{0}$ is chosen to be

$$
c_{0}=\int_{0}^{\infty}\|\nabla \dot{\phi}\|^{2} e^{-t} d t+\frac{1}{V} \int_{X} f_{0} \omega_{0}^{n},
$$

then the constant $\alpha(t)$ is uniformly bounded, and (i) in Theorem 14 implies

$$
\|\dot{\phi}\|_{C^{0}} \leq C \text {. }
$$

8.3.2. Energy functionals and the Kähler-Ricci flow. Recall the energy functionals $F_{\omega}^{0}(\phi), F_{\omega}(\phi)$, and $K_{\omega}(\phi)$ introduced in section $\S 3$. It is wellknown that both energy functionals $F_{\omega}(\phi)$ and $K_{\omega}(\phi)$ decrease along the Kähler-Ricci flow. For the $K$-energy, this is an immediate consequence of its variational definition, and the fact that $\dot{\phi}=-f+\alpha$, where $f$ is the Ricci potential,

(8.21) $\frac{d}{d t} K_{\omega}(\phi)=-\frac{1}{V} \int_{X} \dot{\phi}(R-n) \omega_{\phi}^{n}=\frac{1}{V} \int_{X} \dot{\phi} \Delta \dot{\phi} \omega_{\phi}^{n}=-\frac{1}{V} \int_{X}|\nabla \dot{\phi}|^{2} \omega_{\phi}^{n}$.

As for the functional $F_{\omega}(\phi)$, we have, with $W \equiv \int_{X} e^{-\dot{\phi}} \omega_{\phi}^{n}$,

$$
\frac{d}{d t} F_{\omega}(\phi)=\int_{X}\left(\frac{1}{W} e^{-\dot{\phi}}-\frac{1}{V}\right) \dot{\phi} \omega_{\phi}^{n}=\int_{X}\left(\frac{1}{W} e^{-\dot{\phi}}-\frac{1}{V}\right)\left(\dot{\phi}-\log \frac{V}{W}\right) \omega_{\phi}^{n},
$$

which is negative since the integrand is of the form $-(x-y)\left(e^{x}-e^{y}\right) \leq 0$.

Henceforth, we choose the initial value $c_{0}$ for the flow (8.13) to be (8.19), so that the inequality (8.20) holds. The following identity and bounds will also be very useful:

LEMMA 8. (i) The following identity holds along the Kähler-Ricci flow

$$
K_{\omega}(\phi)-F_{\omega}^{0}(\phi)-\frac{1}{V} \int_{X} \dot{\phi} \omega_{\phi}^{n}=C .
$$

(ii) There exists a constant $C$ so that

$$
\left|F_{\omega}(\phi)-K_{\omega}(\phi)\right|+\left|F_{\omega}^{0}(\phi)-K_{\omega}(\phi)\right| \leq C
$$

along the Kähler-Ricci flow.

Proof. From the derivative of $F_{\omega}^{0}(\phi)$ and the definition of the flow, we have

$$
-\frac{d}{d t} F_{\omega}^{0}(\phi)=\frac{1}{V} \int_{X} \dot{\phi} \omega_{\phi}^{n}=\frac{1}{V} \int_{X} \ddot{\phi} \omega_{\phi}^{n}
$$


since $0=\frac{d}{d t}\left(\frac{1}{V} \int_{X} \omega_{\phi}^{n}\right)=\frac{d}{d t}\left(\frac{1}{V} \int_{X} e^{f_{0}-\phi+\dot{\phi}} \omega_{0}^{n}\right)=-\frac{1}{V} \int_{X} \dot{\phi} \omega_{\phi}^{n}+\frac{1}{V} \int_{X} \ddot{\phi} \omega_{\phi}^{n}$.

On the other hand,

$$
\begin{aligned}
\frac{1}{V} \int_{X} \ddot{\phi} \omega_{\phi}^{n} & =\frac{d}{d t}\left(\frac{1}{V} \int_{X} \dot{\phi} \omega_{\phi}^{n}\right)-\frac{1}{V} \int_{X} \dot{\phi} \Delta \dot{\phi} \omega_{\phi}^{n} \\
& =\frac{d}{d t}\left(\frac{1}{V} \int_{X} \dot{\phi} \omega_{\phi}^{n}\right)+\frac{1}{V} \int_{X}|\nabla \dot{\phi}|^{2} \omega_{\phi}^{n} \\
& =\frac{d}{d t}\left(\frac{1}{V} \int_{X} \dot{\phi} \omega_{\phi}^{n}\right)-\frac{d}{d t} K_{\omega}(\phi) .
\end{aligned}
$$

This establishes (i). Since $|\dot{\phi}|$ is bounded by Perelman's results, $\mid F_{\omega}^{0}(\phi)-$ $K_{\omega}(\phi) \mid$ is bounded. Finally, consider the difference between $F_{\omega}^{0}(\phi)$ and $F_{\omega}(\phi)$,

$$
\left|\log \left(\frac{1}{V} \int_{X} e^{f_{0}-\phi} \omega_{0}^{n}\right)\right|=\left|\log \left(\frac{1}{V} \int_{X} e^{-\dot{\phi}} \omega_{\phi}^{n}\right)\right| \leq C \frac{1}{V} \int_{X} \omega_{\phi}^{n}=C,
$$

and thus $\left|F_{\omega}(\phi)-K_{\omega}(\phi)\right|$ is bounded as well. Q.E.D.

It is convenient to group together the essential inequalities between the quantities $\frac{1}{V} \int_{X} \phi \omega_{0}^{n}, \frac{1}{V} \int_{X}(-\phi) \omega_{\phi}^{n}$, and $J_{\omega}(\phi)$ in the following lemma. Note that the first inequality is the analogue for the Kähler-Ricci flow of the first inequality in (8.6) for the complex Monge-Ampère equation:

Lemma 9. There exists constants $C$ so that

$$
\begin{aligned}
& \frac{1}{n} \frac{1}{V} \int_{X}(-\phi) \omega_{\phi}^{n}-C \leq J_{\omega}(\phi) \leq \frac{1}{V} \int_{X} \phi \omega_{0}^{n}+C \\
& \frac{1}{V} \int_{X} \phi \omega_{0}^{n} \leq n \frac{1}{V} \int_{X}(-\phi) \omega_{\phi}^{n}-(n+1) K_{\omega}(\phi)+C .
\end{aligned}
$$

uniformly along the Kähler-Ricci flow.

Proof. Since $F_{\omega}(\phi)$ is monotone decreasing along the Kähler-Ricci flow, we have $F_{\omega}(\phi) \leq 0$. As noted in the proof of Lemma 8, this implies that $F_{\omega}^{0}(\phi) \leq C$. Now let $I_{\omega}(\phi)$ be the following functional,

$$
I_{\omega}(\phi)=\frac{1}{V} \int_{X} \phi\left(\omega^{n}-\omega_{\phi}^{n}\right)=\frac{i}{2} \sum_{k=0}^{n-1} \int_{X} \partial \phi \wedge \bar{\partial} \phi \wedge \omega^{n-1-k} \wedge \omega_{\phi}^{k} .
$$

Comparing with the expression for $J_{\omega}(\phi)$ in $\S 3.3 .2$, we readily see that

$$
0 \leq \frac{1}{n} J_{\omega}(\phi) \leq \frac{1}{n+1} I_{\omega}(\phi) \leq J_{\omega}(\phi) .
$$

Furthermore, the functional $F_{\omega}^{0}(\phi)$ can be written in terms of $I_{\omega}(\phi)$ and $J_{\omega}(\phi)$ in two different ways,

$$
F_{\omega}^{0}(\phi)=J_{\omega}(\phi)-\frac{1}{V} \int_{X} \phi \omega^{n}=-\left[\left(I_{\omega}-J_{\omega}\right)(\phi)+\frac{1}{V} \int_{X} \phi \omega_{\phi}^{n}\right] .
$$


Using the first way of writing $F_{\omega}^{0}(\phi)$, we obtain the inequality on the right of the first statement of Lemma 9. Using the second way of writing $F_{\omega}^{0}(\phi)$, we obtain

$$
\frac{1}{V} \int_{X}(-\phi) \omega_{\phi}^{n} \leq\left(I_{\omega}-J_{\omega}\right)(\phi)+C \leq n J_{\omega}(\phi)+C .
$$

and the inequality on the left of the first statement of Lemma 9 also follows. The second statement of Lemma 9 is an easy consequence of Lemma 8. The inequality $F_{\omega}^{0}(\phi)-K_{\omega}(\phi) \geq-C$ can be rewritten as

$$
\frac{1}{V} \int_{X} \phi \omega_{0}^{n} \leq J_{\omega}(\phi)-K_{\omega}(\phi)+C \leq \frac{n}{n+1} I_{\omega}(\phi)-K_{\omega}(\phi)+C,
$$

and expressing $I_{\omega}(\phi)$ by its definition gives the desired result. Q.E.D.

The following lemma can be found in Rubinstein [119]. It is the analogue of the second inequality in (8.6) for the complex Monge-Ampère equation. As in that case, it is Moser iteration, applied originally by Yau [157] to the Monge-Ampère equation in his proof of the Calabi conjecture, and subsequently used in the method of continuity for the case of positive $c_{1}(X)$ by $[143,148]$. Its key ingredient is a uniform Sobolev constant, which has now become available for the Kähler-Ricci flow thanks to Theorem 14:

LEMMA 10. We have the following estimate along the Kähler-Ricci flow,

$$
\operatorname{osc} \phi \leq A \frac{1}{V} \int_{X} \phi \omega_{0}^{n}+B \text {. }
$$

Proof. Let $\psi=\max _{X} \phi-\phi+1 \geq 1$. Then for any $\alpha \geq 0$, we have

$$
\begin{aligned}
\int_{X} \psi^{\alpha+1} \omega_{\phi}^{n} & \geq \int_{X} \psi^{\alpha+1}\left(\omega_{\phi}^{n}-\omega_{0} \wedge \omega_{\phi}^{n-1}\right)=\frac{i}{2}(\alpha+1) \int_{X} \psi^{\alpha} \partial \psi \wedge \bar{\partial} \psi \wedge \omega_{\phi}^{n-1} \\
(8.35) & =\frac{i(\alpha+1)}{2\left(\frac{\alpha}{2}+1\right)^{2}} \int_{X} \partial\left(\psi^{\frac{\alpha}{2}+1}\right) \wedge \bar{\partial}\left(\psi^{\frac{\alpha}{2}+1}\right) \wedge \omega_{\phi}^{n-1}
\end{aligned}
$$

Thus we obtain

$$
\left\|\nabla\left(\psi^{\frac{\alpha}{2}+1}\right)\right\| \leq \frac{n\left(\frac{\alpha}{2}+1\right)^{2}}{\alpha+1} \int_{X} \psi^{\alpha+1} \omega_{\phi}^{n},
$$

and hence, in view of Theorem 14, (iv) and setting $\beta \equiv \frac{n}{n-1}>1, p=\alpha+2$,

$$
\left[\int_{X} \psi^{p \beta}\right]^{\frac{1}{\beta}} \leq C p \int_{X} \psi^{p} \omega_{\phi}^{n}, \quad p \geq 2 .
$$

Taking $p=2$ and iterating, $p \rightarrow p \beta \rightarrow \cdots p \beta^{k}$, it follows that

$$
\log \|\psi\|_{L^{\infty}\left(\omega_{\phi}\right)} \leq \sum_{k=1}^{\infty} \frac{\log \left(2 C \beta^{k}\right)}{2 \beta^{k}}+\log \|\psi\|_{L^{2}\left(\omega_{\phi}\right)}=C_{1}+\log \|\psi\|_{L^{2}\left(\omega_{\phi}\right)}
$$


On the other hand, a simple Bochner-Kodaira argument shows that

$$
\frac{1}{V} \int_{X} \psi^{2} e^{f} \omega_{\phi}^{n} \leq \frac{1}{V} \int_{X}|\nabla \psi|^{2} e^{f} \omega_{\phi}^{n}+\left(\frac{1}{V} \int_{X} \psi e^{f} \omega_{\phi}^{n}\right)^{2}
$$

since $R_{\bar{k} j}-g_{\bar{k} j}=\partial_{j} \partial_{\bar{k}} f$ (see $[\mathbf{5 9}]$, or $[\mathbf{1 4 8}, \mathbf{1 0 5}]$ ). By Theorem 14, (i), the measures $e^{f} \omega_{\phi}^{n}$ and $\omega_{\phi}^{n}$ are equivalent. Together with (8.36) with $\alpha=0$, we find that

$$
\left[\frac{1}{V} \int_{X} \psi^{2} \omega_{\phi}^{n}\right]^{\frac{1}{2}} \leq C\left(1+\frac{1}{V} \int_{X} \psi \omega_{\phi}^{n}\right) .
$$

Finally, up to an additive constant, the expression on the right hand side can clearly be bounded by $\sup _{X} \phi$ and $\frac{1}{V} \int_{X}(-\phi) \omega_{\phi}^{n}$. Both of these expressions are bounded, up to an additive constant, by $\frac{1}{V} \int_{X} \phi \omega_{0}^{n}$, the first because of the plurisubharmonicity property $\omega_{0}+\frac{i}{2} \partial \bar{\partial} \phi>0$, and the second in view of Lemma 9. Q.E.D.

LEMmA 11. Let $\left(X, \omega_{0}\right)$ be a compact Kähler manifold, $\omega_{0} \in c_{1}(X)$, and consider the Kähler-Ricci flow (8.13), with initial value $c_{0}$ given by (8.19). If there exists a constant $C$ with

$$
\sup _{t \in[0, \infty)} \frac{1}{V} \int_{X} \phi \omega_{0}^{n} \leq C<\infty,
$$

then the Kähler-Ricci flow converges exponentially fast in $C^{\infty}$ to a KählerEinstein metric.

ProOF. By Lemma 10, the hypothesis implies that the oscillation osc $\phi$ is uniformly bounded. But since $\int_{X} \omega_{\phi}^{n}=1$ and $\|\dot{\phi}\|_{C^{0}}$ is bounded, we also have

$$
0<C_{1} \leq \frac{1}{V} \int_{X} e^{-\phi} \omega_{0}^{n} \leq C_{2}
$$

which implies that

$$
\inf _{X} \phi \leq-\log C_{1}, \quad \sup _{X} \phi \geq-\log C_{2} .
$$

Combined with the bound for osc $\phi$, this implies that $\|\phi\|_{C^{0}}$ is bounded. As in Yau's proof of the Calabi conjecture [157], the $C^{0}$ bound for $\phi$ implies a uniform bound for $\|\phi\|_{C^{k}}$, for each $k \in \mathbf{N}$. Detailed derivations in the case of the Kähler-Ricci flow can be found in $[\mathbf{1 0 3 , 9 9 , 2 2 ]}$. This already implies that there exist subsequences of times $t_{m} \rightarrow+\infty$ with $g_{\bar{k} j}\left(t_{m}\right)$ converging to a Kähler-Einstein metric. The proof that the full flow $g_{\bar{k} j}(t)$ converges exponentially fast to a Kähler-Einstein metric is more involved, and actually makes use of bounds for the lowest eigenvalue $\lambda_{\omega(t)}$ for the operator $\bar{\partial}^{\dagger} \bar{\partial}$ introduced earlier in section $§ 2.6 .2$. It can be found in the second part of the proof of Lemma 6 in $[\mathbf{1 0 5}]$. 
8.3.3. Perelman's convergence theorem. We are now in position to give a proof, different from the earlier one in Tian-Zhu [148], of the following version of a result announced by Perelman in private communications:

THEOREM 15. If $X$ admits a Kähler-Einstein metric $\omega_{K E}$ and $\operatorname{Aut}^{0}(X)=$ 0 , then for any initial metric $\left(g_{0}\right)_{\bar{k} j}$, the Kähler-Ricci flow converges to a Kähler-Einstein metric. More generally, if $\operatorname{Aut}^{0}(X) \neq 0$ and $G \subset \operatorname{Stab}\left(\omega_{K E}\right)$ is a closed subgroup whose centralizer in the stabilizer $\operatorname{Stab}\left(\omega_{K E}\right)$ of $\omega_{K E}$ is finite, then the Kähler-Ricci flow converges to a Kähler-Einstein metric for all G-invariant initial metrics $\left(g_{0}\right)_{\bar{k} j}$.

Proof. Under the hypotheses of the theorem, the Moser-Trudinger inequality (7.1) holds. Since $F_{\omega}(\phi)$ is decreasing under the flow, it follows that $J_{\omega}(\phi)$ is uniformly bounded. The bound (7.1) also shows that $F_{\omega}(\phi)$ is bounded from below. By Lemma $8, K_{\omega}(\phi)$ is then bounded. By the second statement in Lemma $9, \frac{1}{V} \int_{X} \phi \omega_{0}^{n}$ is bounded from above. By Lemma 11, the Kähler-Ricci flow converges. Q.E.D.

We note that by an early result of Bando-Mabuchi [8], Kähler-Einstein metrics are unique up to diffeomorphisms. An extension of Theorem 15 of the case of Kähler-Ricci solitons has been given recently in [148], using an extension of works of Kolodziej [79]. When restricted to the Kähler-Einstein case, $[\mathbf{1 4 8}]$ also yields a proof of Theorem 15 .

8.3.4. Condition $(B)$. There has been so far relatively few results on the convergence of the Kähler-Ricci flow when $c_{1}(X)>0$. The first such result is due to Hamilton [70], who showed convergence for $X=\mathbf{C P}^{1}$, when the initial metric $\left(g_{0}\right)_{\bar{k} j}$ has positive curvature everywhere. This assumption was removed later by B. Chow [35], who showed that, for any initial metric $\left(g_{0}\right)_{\bar{k} j}$, the curvature eventually becomes positive everywhere. Convergence in higher dimensions under the assumption of positive bisectional curvature is treated in Chen and Tian $[\mathbf{3 2}, \mathbf{3 3}]$. This assumption is preserved under the Kähler-Ricci flow $[\mathbf{6 , 9 5}$, but it is restrictive, since it implies that $X$ is holomorphically equivalent to $\mathbf{C P}^{N}[\mathbf{9 6}, \mathbf{1 2 6}]$. In fact, the arguments in $[32,33]$ rely on the existence a priori of a Kähler-Einstein metric. More recently, convergence in the case of toric varieties with vanishing Futaki invariant has been established by Zhu [168], and, as we have seen above, for general manifolds manifolds $X$ under the assumption that $X$ admits a Kähler-Einstein metric (see Theorem 15 above) or Kähler-Ricci soliton [148]. Since toric manifolds with vanishing Futaki invariant are known to admit Kähler-Einstein metrics [154], all these results turn out to require manifolds for which the existence of such metrics were known in advance.

In view of the conjecture of Yau, the convergence of the Kähler-Ricci flow should be tied with the stability of $X$ in the sense of GIT. We describe next some recent results in this direction, where the stability conditions involved are the conditions $(\mathrm{B})$ and $(\mathrm{S})$ described in section $\S 2$. The following theorem was proved in [112]: 
Theorem 16. Let $(X, \omega)$ be a compact Kähler manifold with $c_{1}(X)>0$. Then the Kähler-Ricci flow converges if and only if the Riemann curvature tensor is bounded along the flow, the K-energy is bounded from below, and Condition (B) holds.

We have stated the theorem as it was stated in $[\mathbf{1 1 2}]$. But clearly, the condition (B) can be replaced by the weaker condition (B)* defined in section $\S 2.6 .1$, since in the proof of Theorem 16, only metrics along the Kähler-Ricci flow are considered, and their $K$-energy decreases along the flow and hence is bounded above.

We discuss briefly the role of Condition (B) in the proof of Theorem 16 . Note that the assumption of uniform boundedness of the curvature, together with the fixed volume and the boundedness of the diameter by Perelman's results, implies already that there exist subsequences $g_{\bar{k} j}\left(t_{m}\right)$ which converge in the sense of Cheeger-Gromov, that is, after suitable reparametrizations depending on $t_{m}$. The issue is full convergence, and more important, the convergence of $g_{\bar{k} j}(t)$ as a sequence of tensors, point by point on $X$.

Now the assumptions that the $K$-energy is bounded from below and the Riemann curvature tensor is bounded imply that $\left\|\dot{g}_{\bar{k} j}\right\|_{C^{k}}=\left\|R_{\bar{k}_{j}}-g_{\bar{k} j}\right\|_{C^{k}}$ tends to 0 for all $k$, but where norms are taken with respect to the evolving metric $g_{\bar{k} j}(t)$. The main step is then to show that the metrics $g_{\bar{k} j}(t)$ are uniformly equivalent. For this, according to a lemma of Hamilton [68], it suffices to establish an exponential decay for $\left\|\dot{g}_{\bar{k} j}\right\|_{t}$. The key starting point is the following differential inequality $([\mathbf{1 1 2}]$, eq. $(3.7))$ for $Y(t)$ defined by

$$
Y(t)=\int_{X}|\nabla f|^{2} \omega_{\phi}^{n}
$$

where $f$ is the Kähler-Ricci potential defined by (2.4),

$$
\begin{aligned}
\dot{Y}(t) \leq & -2 \lambda_{t} Y(t)+2 \lambda_{t} \operatorname{Fut}\left(\pi_{t}\left(\nabla^{j} f\right)\right)-\int_{X}|\nabla f|^{2}(R-\mu n) \omega^{n} \\
& -\int_{X} \nabla^{j} f \overline{\nabla^{k} f}\left(R_{\bar{k} j}-g_{\bar{k} j}\right) \omega_{\phi}^{n} .
\end{aligned}
$$

Here $\pi_{t}$ is the orthogonal projection of $(1,0)$-vector fields onto the space of holomorphic vector fields, Fut $(V)$ is the Futaki invariant acting on $V \in$ $H^{0}\left(X, T^{1,0}(X)\right)$, and $\lambda_{t}$ is the lowest strictly positive eigenvalue of the $\bar{\partial}^{\dagger} \bar{\partial}$ operator on vector fields. Under our assumptions, all the terms on the right hand side of (8.45) tend to 0 except for the term $-2 \lambda_{t} Y(t)$. To obtain exponential decay for $Y(t)$, we shall produce a strictly positive lower bound for $\lambda_{t}$.

This follows from Condition (B): assume otherwise. Since the curvatures, volume, diameter of the metrics $g(t) \equiv g_{\bar{k} j}(t)$ are bounded above and their injectivity radii bounded from below, by the Cheeger-GromovHamilton compactness theorem $[\mathbf{2 5}, \mathbf{6 1}, \mathbf{7 1}]$, a subsequence $F_{t_{j}}^{*}\left(g\left(t_{j}\right)\right)$ converges in $C^{\infty}$, after suitable reparametrizations $F_{t_{j}}$. The eigenvalues of the 
$\bar{\partial}^{\dagger} \bar{\partial}$ operator with respect to the metric $F_{t_{j}}^{*}\left(g\left(t_{j}\right)\right)$ and the almost-complex structure $F_{t_{j}}(J)$ are the same as $\lambda_{t_{j}}$. By going to a subsequence if necessary, we may assume that $F_{t_{j}}(J)$ converges to an almost-complex structure $J_{\infty} \in \overline{\operatorname{Diff}(X) \cdot J}$. But $\lambda_{t} \rightarrow 0$, and thus $\operatorname{dim} H^{0}\left(X, T_{J_{\infty}}^{1,0}(X)\right)>$ $\operatorname{dim} H^{0}\left(X, T_{J}^{1,0}(X)\right)$, contradicting Condition (B).

Once the exponential decay of $Y(t)=\|\nabla f\|_{(0)}^{2}$ is established, repeated applications of Bochner-Kodaira formulas show that $\|\nabla f\|_{(s)}$ converge exponentially to 0 for all $s$. This implies the exponential convergence to 0 of $\left\|\dot{g}_{\bar{k} j}(t)\right\|_{C^{0}}$, in the $g_{\bar{k} j}(t)$ norm, which implies the uniform equivalence of all metrics $g_{\bar{k} j}(t)$, by the lemma of Hamilton [68]. Once the uniform equivalence of $g_{\bar{k} j}(t)$ is established, the proof of $C^{\infty}$ convergence is easy.

The method of integral estimates has also been applied successfully by $\mathrm{Hou}$ and $\mathrm{Li}[\mathbf{7 4}]$ to boundary value problems for real Monge-Ampère equations.

8.3.5. Condition (S). One advantage of Condition (B) is that it is clearly a necessary condition for the orbit of $J$ to be a Hausdorff point in the moduli space of orbits of almost-complex structures. However, the assumption of uniform boundedness of the Riemann curvature tensor along the KählerRicci flow in Theorem 16 is very restrictive. The following theorem due to [105] eliminates the curvature assumption completely, by replacing Condition (B) by the closely related Condition (S):

Theorem 17. (i) If $\inf _{\omega \in \pi c_{1}(X)} K_{\omega_{0}}(\omega)>-\infty$, and Condition (S) holds, then the Kähler-Ricci flow $g_{\bar{k} j}(t)$ converges exponentially fast to a KählerEinstein metric.

(ii) Conversely, if the metrics $g_{\bar{k} j}(t)$ converge in $C^{\infty}$ to a Kähler-Einstein metric, then the above two conditions are satisfied.

(iii) In particular, if $g_{\bar{k} j}(t)$ converge in $C^{\infty}$, then the convergence is exponential.

We sketch the proof of the convergence of the flow under the assumptions in the theorem. It depends on the following criterion for the convergence of the flow $[\mathbf{1 0 5}]$ :

LEMMA 12. If the following inequality is satisfied,

$$
\int_{0}^{\infty}\|R-n\|_{C^{0}} d t<\infty
$$

then the Kähler-Ricci flow converges exponentially fast in $C^{\infty}$ to a KählerEinstein metric.

Indeed, by definition of the Kähler-Ricci flow, $\partial_{t} \log \left(\omega_{\phi}^{n} / \omega_{0}^{n}\right)=-(R-$ $n)$, and the condition in the lemma implies that the volume forms $\omega_{\phi}^{n}$ are all equivalent in size to the volume form $\omega_{0}^{n}$. But then $\phi=-\log \left(\omega_{\phi}^{n} / \omega_{0}^{n}\right)+$ $\dot{\phi}+f_{0}$ is bounded in $C^{0}$, and we can apply Lemma 11 . 
Returning to the proof of the theorem proper, the first important step is to show that, under the sole assumption that the $K$-energy is bounded from below, we have

$$
\|R(t)-n\|_{C^{0}} \rightarrow 0 \quad \text { as } t \rightarrow+\infty
$$

This is the analogue for the Kähler-Ricci flow of an estimate established by Bando [7] for the method of continuity. Combined with the inequality (8.45), this leads to the following key difference-differential inequality for $Y(t)=\|\nabla f\|_{L^{2}}^{2}$,

$$
\dot{Y}(t) \leq-2 \lambda_{t} Y+\epsilon \prod_{j=0}^{N} Y(t-j)^{\frac{\delta_{j}}{2}}, \quad \sum_{j=1}^{N} \delta_{j}=2, \quad \delta_{j}>0,
$$

for a fixed integer $N$, any given $\epsilon>0$, and $t \in\left[T_{\epsilon}, \infty\right)$, for a suitable $T_{\epsilon}$ large enough. The point is that a difference-differential inequality of this form can still guarantee the exponential decay of $Y(t)$. In fact, let $Z(t)=Z_{0} e^{-\mu\left(t-T_{\epsilon}\right)}$, for some $0<\mu<1$, and take $\epsilon=\frac{1}{2} \inf _{t} \lambda_{t}>0$. We claim that, for $Z_{0}$ large enough and $\mu$ small enough, $Z(t)$ is a barrier for $Y(t)$. Indeed, write $T_{\varepsilon}=0$ for simplicity, and let $Z_{0}$ be chosen so that $Y(t)<Z(t)$ for $t \in[0, N]$. We claim that $Y(t)<Z(t)$ for all $t$. Otherwise, let $T$ be the first time with $Y(T)=Z(T)$. Then $\dot{Y}(T) \geq \dot{Z}(T)$. On the other hand,

$$
\begin{aligned}
\dot{Y}(T)-\dot{Z}(T) & \leq-2 \varepsilon Y(T)+\mu Z(T)+\varepsilon \prod_{j=0}^{N} Y(T-j)^{\delta_{j}} \\
& \leq Z_{0}\left\{(\mu-2 \epsilon) e^{-\mu T}+\varepsilon e^{-\mu T+\mu \sum_{j=0}^{N} j \delta_{j}}\right\}<0
\end{aligned}
$$

for $\mu$ small enough, depending only on $\varepsilon, N$, and $\delta_{j}$. This is a contradiction, and thus we do have $Y(t)<Z(t)=Z_{0} e^{-\mu t}$ for all $t \geq 0$. Combined with Futaki's $L^{2}\left(e^{f} \omega^{n}\right)$ Poincare inequality [59] and Perelman's non-collapse theorem, the exponential decay of $Y(t)=\|\nabla f\|_{L^{2}}^{2}$ can be shown to imply an exponential decay for $\|f\|_{C^{0}}$, and ultimately for $\|\Delta f-n\|_{C^{0}}=\|R-n\|_{C^{0}}$, and the theorem follows.

Several extensions and applications of Theorems 16 and 17 have been obtained in [106]. In particular, the condition that the $K$-energy be bounded from below in Theorem 16 can be weakened to just the vanishing of the Futaki invariant. It is also shown in [106] that, under the assumption that the $\mathrm{K}$ energy is bounded from below (or just the vanishing of the Futaki invariant when $\operatorname{dim} X \leq 2$ ), if the initial metric has positive bisectional curvature, then the Kähler-Ricci flow converges to a Kähler-Einstein metric. We stress that the convergence of the Kähler-Ricci flow has been treated in $[\mathbf{3 2}, \mathbf{3 3}]$, but the arguments there rely on the existence of a Kähler-Einstein metric, and thus on the solution of the Frankel conjecture. Here it is essential that the arguments do not assume the a priori existence of such a metric, in order 
to be viewed as a progress in the problem of giving an independent proof of the Frankel conjecture by flow methods.

8.3.6. Multiplier ideal sheaves. We have seen in section $\S 8.2$ how multiplier ideal sheaves arise from the continuity method for the complex MongeAmpère equation. Here we discuss the adaptation of such ideas to the context of the Kähler-Ricci flow.

THEOREM 18. Consider the Kähler-Ricci flow (8.13) on a compact Kähler manifold $\left(X, \omega_{0}\right)$, with $\omega_{0} \in c_{1}(X)$, and initial value $c_{0}$ given by (8.19). Then the Kähler-Ricci flow converges if and only if there exists $p>1$ so that

$$
\sup _{t \geq 0} \frac{1}{V} \int_{X} e^{-p \phi} \omega_{0}^{n}<\infty
$$

The convergence is then in $C^{\infty}$ and exponentially fast.

This theorem is proved in [103], using a priori estimates of Kolodziej [79, 80] for Monge-Ampère equations with $L^{p}$ right hand sides, $p>1$. (In [103], the proof of the full convergence of the flow, by opposition to convergence only along a subsequence of times $t_{m} \rightarrow+\infty$, was given only under the assumption that $\operatorname{Aut}^{0}(X)=0$. But we now have at our disposal Lemma 11, based on the recent improvements in [105] for convergence arguments, and this technical assumption can be removed.) Clearly, Theorem 18 can be restated in terms of a version of multiplier ideal sheaves: let $\mathcal{J}^{p}$ be the sheaf whose stalk $\mathcal{J}_{z}^{p}$ at $z \in X$ is defined by

$$
\mathcal{J}_{z}^{p}=\left\{f ; \quad \exists U \ni z, f \in \mathcal{O}(U) \quad \sup _{t \geq 0} \int_{U} e^{-p \phi}|f|^{2} \omega_{0}^{n}<\infty\right\}
$$

Then the necessary and sufficient condition for the convergence of the KählerRicci flow is that there exists $p>1$ so that $\mathcal{J}^{p}$ admits the global section 1 .

An application of Theorem 18 to del Pezzo surfaces can be found in [72].

Theorem 18 implies the following weaker, but simpler statement. First note that for any $p>1$ we have

$$
\sup _{t \geq 0} \frac{1}{V} \int_{X} e^{-p \phi} \omega_{0}^{n}=\infty \Rightarrow \sup _{t \geq 0} \frac{1}{V} \int_{X} e^{-p\left(\phi-\frac{1}{V} \int_{X} \phi \omega_{0}^{n}\right)} \omega_{0}^{n}=\infty .
$$

In fact, replacing $\phi \rightarrow \phi-\sup _{X} \phi$ in the integrals on the left hand side only increases their sizes, in view of the estimate (8.43). But $\sup _{X} \phi \leq$ $\frac{1}{V} \int_{X} \phi \omega_{0}^{n}+C$ by $\omega_{0}$-plurisubharmonicity, hence the assertion. Assume now that a Kähler-Einstein metric does not exist, so that for all $p>1$, there exists a subsequence of times $t_{m} \rightarrow+\infty$. Let $\psi$ be a weak limit of $\phi-\frac{1}{V} \int_{X} \phi \omega_{0}^{n}$. Then the multiplier ideal sheaf $\mathcal{I}(p \psi)$ defined as in (8.2) defines a proper, coherent sheaf, with $H^{q}\left(X, K_{X}^{-[p]} \otimes \mathcal{I}^{p}\right)=0, q \geq 1$.

This last statement has been recently strengthened by Rubinstein [119] to the same range $p \in\left(\frac{n}{n+1}, \infty\right)$ that Nadel $[\mathbf{9 8}]$ established for the multiplier ideal sheaves obtained from the method of continuity. The point is that, together with Lemmas 9, 10 and the following Lemma 13, we have now the analogues in the case of the Kähler-Ricci flow of all the ingredients 
required for Nadel's arguments in the method of continuity, namely (8.6) and Lemma 7:

Lemma 13. The exact same statement as in Lemma 7 holds, with $\phi$ the solution of the Kähler-Ricci flow (8.13).

Proof. As in the proof of Lemma 7, the concavity of the logarithm implies

$$
C>\frac{1}{V} \int_{X} e^{(1-p) \phi-f_{0}+\dot{\phi}} \omega_{\phi}^{n} \geq \frac{1}{V} \int_{X}\left((1-p) \phi-f_{0}+\dot{\phi}\right) \omega_{\phi}^{n}+p \frac{1}{V} \int_{X} \phi \omega_{0}^{n},
$$

and thus, by Perelman's uniform bound for $|\dot{\phi}|$ and Lemma 9,

$$
p \frac{1}{V} \int_{X} \phi \omega_{0}^{n} \leq(1-p) \frac{1}{V} \int_{X}(-\phi) \omega_{\phi}^{n}+C \leq n(1-p) \frac{1}{V} \int_{X} \phi \omega_{0}^{n}+C .
$$

and Lemma 13 follows as before [119]. Q.E.D.

Assume now that $X$ does not admit a Kähler-Einstein metric. Then $\frac{1}{V} \int_{X} \phi \omega_{0}^{n}$ must diverge to $+\infty$ for some subsequence of times $t_{m} \rightarrow+\infty$, for otherwise Lemma 11 would imply that the Kähler-Ricci flow converges to a Kähler-Einstein metric. Thus the integrals in (8.7) must diverge to $\infty$ for some $p \in\left(\frac{n}{n+1}, 1\right]$. If we let $\psi$ be an $L^{1}$ limit point of $\phi-\frac{1}{V} \int_{X} \phi \omega_{0}^{n}$, then $\mathcal{I}(p \psi)$ provides the desired coherent, acyclic multiplier ideal sheaf.

\section{General L: Energy functionals and Chow points}

With this section, we begin the description of results which address, at least partially in some way, the eventual sufficiency of stability conditions for constant scalar curvature metrics in a general Kähler class $L$, which is not necessarily $K_{X}^{-1}$. A first class of results links directly the energy functionals $K_{\omega}(\phi)$ and $F_{\omega}^{0}(\phi)$ to the Chow point. That there should be some relation is to some extent already built into the notion of stability: we have seen that $K_{\omega}(\phi)$ and $F_{\omega}^{0}(\phi)$ are just the conformal changes of metrics in the very Deligne pairings which define the line bundles $\eta_{K}$ and $\eta_{C h o w}$ over the Hilbert scheme giving the notions of $\mathrm{K}$ and Chow-Mumford stability. But the direct relations which we describe below are much more precise, and one can hope that, combined with some suitable $k \rightarrow \infty$ limiting process, they may eventually allow to deduce the asymptotic growth of $K_{\omega}(\phi)$ and $F_{\omega}^{0}(\phi)$ on $\mathcal{K}$ from stability conditions.

9.1. $\boldsymbol{F}_{\boldsymbol{\omega}}^{\mathbf{0}}$ and Chow points. We start with the case of $F_{\omega}^{0}$. Here the basic result is the following theorem due to Zhang [166], which links all three concepts of critical points for $F_{\omega}^{0}(\phi)$, balanced imbeddings, and ChowMumford stability:

THEOREM 19. Let $\hat{X} \subset \mathbf{C P}^{N}$ be a smooth projective variety. Let $\omega=$ $\omega_{F S}, \phi_{\sigma}(x)=\log \frac{|\sigma x|^{2}}{|x|^{2}}$, and view $F_{\omega}^{0}\left(\phi_{\sigma}\right)$ as a function of $\sigma \in S L(N+1) /$ $S U(N+1)$. Then

(a) $-F_{\omega}^{0}$ is convex along one-parameter subgroups; 
(b) A point $\sigma_{0}$ is a critical point for $F_{\omega}^{0}$ if and only if $\sigma_{0}(\hat{X})$ is balanced, in the sense c.f. (5.11) that

$$
\int_{\sigma(\hat{X})} \frac{\bar{x}_{\alpha} x_{\beta}}{|x|^{2}} \sigma_{F S}^{n} \sim \delta_{\bar{\alpha} \beta} .
$$

(c) Define a norm $\|f\|$ on $f \in H^{0}(G r, O(d))$ by

$$
\log \|f\|^{2}=\frac{1}{D} \int_{G r} \log \frac{|f(z)|^{2}}{|P l(z)|^{2 d}} \omega_{G r}^{m}
$$

where $G r=G r\left(N-n-1, \mathbf{C P}^{N}\right)$ is the Grassmannian of $N-n-1$ planes in $\mathbf{C P}^{N}, P l$ is the Plücker imbedding, $\omega_{G r}$ is the Fubini-Study metric restricted to $G r$, and $D$ is its volume. Then we have

$$
-F_{\omega}^{0}\left(\phi_{\sigma}\right)=\log \frac{\|\sigma \cdot \operatorname{Chow}(\hat{X})\|^{2}}{\|\operatorname{Chow}(\hat{X})\|^{2}} .
$$

(d) In particular, $\hat{X}$ is Chow-Mumford stable if and only if there exists a unique $\sigma_{0} \in S L(N+1) / S U(N+1)$ with $\sigma_{0}(\hat{X})$ balanced.

The statements (a) and (b) and their proofs have been given in section $\S 5$. Zhang's original proof of (c) using Deligne pairings is in [166]. A different and perhaps simpler proof based on comparing the derivatives of both sides along one-parameter subgroups can be found in [107]. We show now how (d) follows from (a), (b), and (c). The theorem of Kempf and Ness [75] says that $\hat{X}$ is stable if and only if $\|\sigma \cdot \operatorname{Chow}(\hat{X})\|^{2}$ is a proper map from $S L(N+1)$ in to $\mathbf{R}$, in the sense that the inverse image of any compact subset is compact. Equivalently, $\hat{X}$ is stable if and only if

(9.4) $\log \|\sigma \cdot \operatorname{Chow}(\hat{X})\|^{2} \geq-C$ and $\lim _{\sigma \rightarrow \infty} \log \|\sigma \cdot \operatorname{Chow}(\hat{X})\|^{2}=\infty$ (where the latter statement means that for any constant $M$, there exists a compact set $K \subset S L(N+1)$ so that $\log \|\sigma \cdot \operatorname{Chow}(\hat{X})\|^{2} \geq M$ for $\sigma \notin K$.)

Assume that $\hat{X}$ is Chow-Mumford stable. In view of the above KempfNess characterization of stability, this implies that $\log \|\sigma \cdot \operatorname{Chow}(\hat{X})\|^{2}$ must attain its mimimum, and $-F_{\omega}^{0}$ must have a critical point $\sigma_{0}$. This critical point is unique, since if there are two distinct critical points, the convexity of $-F_{\omega}^{0}$ along the one-parameter subgroup joining them would force $\log \| \sigma$. Chow $(\hat{X}) \|^{2}$ to be constant along this geodesic, contradicting its properness. By (b), a point $\sigma_{0}$ is a critical point if and only if $\sigma_{0}(\hat{X})$ is balanced.

Conversely, assume that there exists a unique balanced, or equivalently, a unique critical point $\sigma_{0}$. By the convexity of $-F_{\omega}^{0}$ along one-parameter subgroups, this point must be a minimum along any such path. And since any point in $S L(N+1)$ can be joined to $\sigma_{0}$ by such a path, $\sigma_{0}$ must be a minimum for $-F_{\omega}^{0}$ on the whole of $S L(N+1)$. It is a strict minimum since the existence of another minimum would contradict the uniqueness assumption of balanced points. It follows that $\log \|\sigma \cdot \operatorname{Chow}(\hat{X})\|^{2}$ is bounded from below, and must tend to $\infty$ when restricted to any one-parameter subgroup. 
This implies that the numerical invariant along any one-parameter subgroup must be strictly positive, and, in view of the Hilbert-Mumford criterion, the variety $\hat{X}$ must be stable.

9.2. $\boldsymbol{K}_{\boldsymbol{\omega}}$ and Chow points. The analogue for the $K$-energy of the formula of the previous section is the following [107]:

TheOrem 20. Let $X \subset \mathbf{C P}^{N}$ be as in Theorem 19. Then

$$
\begin{aligned}
K_{\omega}\left(\phi_{\sigma}\right) & +\frac{1}{V}\left\langle\left[Y_{s}\right], \Phi_{\sigma} \sum_{i=0}^{m-1} \omega_{Z}^{i} \sigma^{*} \omega_{Z}^{m-1-i}\right\rangle \\
-\frac{D}{V} & \frac{m \operatorname{deg}\left(Y_{s}\right)}{m+1} \log \frac{\|\sigma \cdot \operatorname{Chow}(X)\|^{2}}{\|\operatorname{Chow}(X)\|^{2}} \\
& =\frac{D(m+2)(d-1)}{V(m+1)} \log \frac{\|\sigma \cdot \operatorname{Chow}(X)\|_{\#}^{2}}{\|\operatorname{Chow}(X)\|_{\#}^{2}}
\end{aligned}
$$

Here $Z=\left\{w \in G r\left(N-n-1, \mathbf{C P}^{N}\right) ; w \cap \hat{X} \neq 0\right\}$ is the Chow variety of $X$. In general, $Z$ is a singular variety, and we denote by $Y_{s}=\{w \in$ $\left.\operatorname{Gr}\left(N-n-1, \mathbf{C P}^{N}\right) ; \#(w \cap \hat{X})>1\right\}$ the subvariety where it is singular. We denote by $\omega_{Z}$ the restriction of the Fubini-Study Kähler form $\omega_{G r}$ on the Grassmannian to the regular part $Z \backslash Y_{s}$ of $Z$, and by $\left[Y_{s}\right]$ the current of integration on $Y_{s}$. Let $P \ell: G r\left(N-n-1, \mathbf{C P}^{N}\right) \rightarrow \mathbf{P}\left(\wedge^{N-n} \mathbf{C P}^{N+1}\right)$ be the Plücker imbedding, and set for each $\sigma \in G L(N+1), \phi_{\sigma}(z)=\log \frac{|\sigma z|^{2}}{|z|^{2}}$, $\Phi_{\sigma}(z)=\log \frac{|P \ell(\sigma z)|^{2}}{|P \ell(z)|^{2}}$. The norm $\|\cdot\|$ is the norm defined in Theorem 19, while $\|\cdot\|_{\#}$ is a degenerate semi-norm defined for $f \in H^{0}(G r, O(d))$ as follows

$$
\begin{aligned}
\log \|f\|_{\#}^{2}= & \frac{m+1}{(m+2)(d-1)} \frac{1}{D} \int_{Z} \log \left(\frac{\omega_{G r}^{m} \wedge \partial \bar{\partial} \frac{|f(z)|^{2}}{|P \ell(z)|^{2 d}}}{\omega_{G r}^{m+1}}\right) \omega_{G r}^{m} \\
& +\frac{d-m-2}{(m+2)(d-1)} \frac{1}{D} \int_{G r} \log \frac{|f(z)|^{2}}{|P \ell(z)|^{2 d}} \omega_{G r}^{m+1},
\end{aligned}
$$

with $m=(N-n)(n+1)-1$ and $D$ the dimension and the volume of the Grassmannian. The various ingredients in the formula (9.5) have an interesting interpretation. We can define a $K$-energy $K_{\omega_{Z}}\left(\Phi_{\sigma}\right)$ associated to the regular part $Z \backslash Y_{s}$ of the variety $Z \subset G r$ just like the $K$-energy $K_{\omega}\left(\phi_{\sigma}\right)$ for the variety $X \subset \mathbf{C P}^{N}$. Remarkably, an exact Radon transform argument shows that $K_{\omega_{Z}}\left(\Phi_{\sigma}\right)=K_{\omega}\left(\phi_{\sigma}\right)$. The left hand side of the equation (9.5) can then be viewed as a notion of $K$-energy associated to the full variety $Z$, and it is this modified $K$-energy associated to a singular variety which satisfies an identity analogous to the identity for $F_{\omega}^{0}\left(\phi_{\sigma}\right)$ stated in Theorem 19.

A formula for the $K$-energy in terms of a Quillen norm, up to a bounded error, has also been obtained by Tian [142]. The Futaki ivariant and $K$-energy for hypersurfaces have been evaluated by Lu $[\mathbf{8 6}, \mathbf{8 8}]$, Yotov $[\mathbf{1 6 2}]$, and in [110]. 
We note that (9.5) can be interpreted as a degenerate norm on a suitable line bundle over the Hilbert scheme. More precisely, let $\eta_{C h o w}$ be the Chow line bundle defined before, and define another line bundle $\eta_{C h o w_{s}}$ by associating to each variety $X$ the Chow line of $Y_{s} \subseteq \mathbf{P}\left(\wedge^{N-n} \mathbf{C P}^{N+1}\right)$, equipped with the corresponding norm $\|\cdot\|_{s}$ defined as in (9.2). Then $K$-energy restricted to $S L(N+1)$ orbits is the change in the norm $\left(\|\cdot\| \otimes\|\cdot\|_{\#}\right) \otimes\|\cdot\|_{s}$ on the line bundle

$$
\eta_{\text {Chow }} \otimes \eta_{\text {Chow }}
$$

Besides this formal application, it would be very interesting to explore the full consequences of the formula (9.5), since it relates several basic objects, namely the $K$-energy, the Chow point, the singular locus $Y_{s}$, and a degenerate semi-norm.

\section{General $L$ : the Calabi energy and the Calabi flow}

We have seen that in the case $L=K_{X}^{-1}$, the metrics of constant scalar curvature metrics are Kähler-Einstein metrics, and from the point of view of geometric flows, the problem can be reduced to the issue of convergence of the Kähler-Ricci flow. For general $L$, we need to deal with the full 4th-order equation, the natural parabolic version of which is the Calabi flow, which we discuss briefly in this section.

10.1. The Calabi flow. Let $L \rightarrow X$ be a positive line bundle over a compact complex manifold $X$, and let $\omega_{0}=\frac{i}{2} g_{\bar{k} j}^{0} d z^{j} \wedge d \bar{z}^{k}$ be a Kähler form in $c_{1}(L)$. The Calabi flow is the 4th-order flow defined by the following equation

$$
\dot{g}_{\bar{k} j}=\partial_{\bar{k}} \partial_{j} R, \quad g_{\bar{k} j}(0)=g_{\bar{k} j}^{0} .
$$

Clearly, it preserves the Kähler class of $\omega_{0}$. If we write then $g_{\bar{k} j}=g_{\bar{k} j}^{0}+\partial_{\bar{k}} \partial_{j} \phi$, the Calabi flow is equivalent to the following flow for $\phi$,

$$
\dot{\phi}=R-\bar{R} \text {. }
$$

In view of the fact that the variational derivative of the $K$-energy is $-(R-\bar{R})$ $\omega_{\phi}^{n}$, we see that the Calabi flow is just the gradient flow of the $K$-energy, and that

$$
\frac{d}{d t} K_{\omega_{0}}(\phi)=-\frac{1}{V} C(\phi)
$$

where $C(\phi)$ is the Calabi functional, defined by

$$
C(\phi)=\int_{X}\left|R\left(\omega_{\phi}\right)-\bar{R}\right|^{2} \omega_{\phi}^{n} .
$$

Clearly, metrics of constant scalar curvature are the minima of the Calabi functional $C(\phi)$. However, the other critical points of $C(\phi)$, called "extremal 
metrics", are also of considerable interest. Now a straightforward calculation gives the following variational formula for $C(\phi)$

$$
\delta C=-\int_{X} \delta \phi\left(\Delta^{2} R+|\nabla R|^{2}+R_{\bar{k}_{j}} \nabla^{\bar{k}} \nabla^{j} R\right) \omega_{\phi}^{n} .
$$

Applying the Bianchi inequality, we can easily verify that

$$
\Delta^{2} R+|\nabla R|^{2}+R_{\bar{k} j} \nabla^{\bar{k}} \nabla^{j} R=\nabla^{\bar{l}} \nabla^{\bar{q}} \nabla_{\bar{q}} \nabla_{\bar{l}} R .
$$

Thus the extremal metrics are given by the following equation

$$
\nabla_{\bar{q}} \nabla_{\bar{l}} R=0
$$

which means exactly that $\nabla^{j} R$ is a holomorphic vector field. It follows also immediately from the variational formula for $C(\phi)$ that it decreases along the Calabi flow,

$$
\frac{d}{d t} C(\phi)=-2 \int_{X}\left|\nabla_{\bar{q}} \nabla_{\bar{l}} R\right|^{2} \omega_{\phi}^{n} \leq 0 .
$$

The operator $f \rightarrow \nabla_{\bar{q}} \nabla_{\bar{l}} f$ mapping functions to symmetric two-tensors is the Lichnerowicz operator $\mathcal{D}$ which we have already encountered in section $\S 7.2$. Its central role in the problem of canonical metrics had been stressed by Calabi $[22]$.

When $\operatorname{dim} X=1$, the Calabi flow has been shown to converge to a metric of constant scalar curvature by Chrusciel [38], Chen [26], and Struwe [135]. In higher dimensions, both the long-time existence and the convergence of the Calabi flow are open problems. There has however been several recent progresses. In [29], the flow was shown to exist as long as the Ricci curvature stays uniformly bounded. In [150], the flow was shown to converge if the Calabi functional is initially small enough, and either $c_{1}(X)=0$, or $c_{1}(X)<$ 0 and $c_{1}(L)$ is sufficiently close to $c_{1}(X)$. In the case of ruled surfaces, the long-time existence for initial metrics given by the momentum construction has been established in [64] under the assumption of existence of an extremal metric, and in general in [137].

10.2. Extremal metrics and stability. We have seen that the extremal metrics are the critical points of the Calabi energy, while the metrics of constant scalar curvature are its minima. Just as in the case of constant scalar curvature metrics, the existence of extremal metrics is expected to be equivalent to some suitable form of stability. In [136], a notion of $K$-stability of a line bundle $L \rightarrow X$, relative to a maximal torus of automorphisms, is introduced and conjectured by Szekelyhidi to be equivalent to the existence of an extremal metric in $c_{1}(L)$. We give here a brief description of these ideas.

It is well known that the map $J \mapsto R\left(g_{J}\right)-\bar{R}$ is a moment map for the action of the symplecto-morphism group of $(X, \omega)$ on the space $\mathcal{J}$, consisting of integrable complex structures on $X$ compatible with the symplectic form $\omega$ (here $g_{J}$ is the Kähler form $g_{J}(v, w)=\omega(v, J w)$ ). Thus the Calabi function may be viewed as the norm squared of the moment map and, in order to gain 
some insight into the nature of its critical points, Szekelyhidi first examines the finite-dimensional picture: Let $L \rightarrow(X, \omega)$ be as above and let $K$ be a compact Lie group acting biholomorphically on $L \rightarrow X$ and preserving $\omega$. A moment map for the action of $K$ on $(X, \omega)$ is a smooth $K$-equivariant map $\mu: X \rightarrow \operatorname{Lie}(K)^{*}$ with the property

$$
W(\langle\mu, \xi\rangle)=\omega\left(V_{\xi}, W\right)
$$

for every smooth vector field $W$ and every $\xi \in \operatorname{Lie}(K)$ (here $V_{\xi}$ is the vector field on $X$ generated by $\xi$ ). Let $G$ be the complexification of $K$ and assume $G$ acts as well on $L \rightarrow X$ and that the action is compatible with that of $K$. Recall that an orbit $G x$ is stable if for every one-parameter subgroup $\lambda(t)=\exp (t \alpha)$, the weight $F_{x}(\alpha)=F_{x}(\lambda)$ is positive. We say that the orbit is polystable if $F_{x}(\lambda) \geq 0$ with equality only if $\lambda$ fixes $x$. Then the KempfNess theorem says that a $G$-orbit $G x$ contains a zero of $\mu$ if and only if the orbit is polystable.

The analogue of the Calabi functional in this finite-dimensional setting is the function $c=\|\mu\|^{2}: X \rightarrow[0, \infty$ ) (the norm is taken with respect to a fixed inner product $($,$) on Lie (K)$ ), and extremal metrics correspond to critical points of the function $c(x)$. The following generalization of the Kempf-Ness theorem is proved in $[\mathbf{1 3 6}]$ :

TheOREM 21. A point $x \in X$ is in the $G$-orbit of a critical point of $c$ if and only if it is polystable relative to a maximal torus in $G_{x}$, where $G_{x}$ is the stabilizer of $x$.

Let us explain the terminology of the theorem: Let $T \subseteq G_{x}$ be a maximal torus and let $G_{T}$ be the connected component of the centralizer of $T$ (elements of $G$ which commute with all the elements of $T$ ). Then there is a connected subgroup $G_{T^{\perp}} \subseteq G_{T}$ which can be characterized as follows: it is isomorphic to $G / T$ under the map $G_{T^{\perp}} \hookrightarrow G_{T} \rightarrow G_{T} / T$, and it has the property that $(\alpha, \beta)=0$ for all $\alpha \in \operatorname{Lie}(T)$ and all $\beta \in \operatorname{Lie}\left(G_{T^{\perp}}\right)$. We say that $x \in X$ is polystable relative to $T$ if it is polystable for the action of $G_{T^{\perp}}$ on $(X, L)$, that is, if and only if

$$
F_{x}(\alpha) \geq 0 \text { for all } \alpha \in \operatorname{Lie}\left(G_{T^{\perp}}\right) \text { with equality if and only if } \lambda_{\alpha} \text { fixes } x \text {. }
$$

One can rewrite condition (10.10) as a condition on the set of all oneparameter subgroups $G_{T}$ as follows: consider the linear map $\operatorname{Lie}(T) \rightarrow \mathbf{R}$ given by $\alpha \mapsto F_{x}(\alpha)$ where $\alpha \in \operatorname{Lie}(T)$, and let $\chi \in \operatorname{Lie}(T)$ be its dual. Thus $F_{x}(\alpha)=(\alpha, \chi)$ for all $\alpha \in \operatorname{Lie}(T)$. Let $F_{x, \chi}(\alpha)=F_{x}(\alpha)-(\alpha, \chi)$. Then one shows that $(10.10)$ can be rewritten as follows

$$
F_{x, \chi}(\alpha) \geq 0 \text { for all } \alpha \in \operatorname{Lie}(K) \cap \operatorname{Lie}\left(G_{T}\right)
$$

with equality if and only if $\alpha$ fixes $x$.

To generalize these notions to the infinite-dimensional setting, Szekelyhidi first constructs an inner product on the space of test configurations: Let $V$ be a projective scheme and $L \rightarrow V$ a very ample line bundle. 
A $\mathbf{C}^{\times}$action for $L \rightarrow V$ is a homomorphism $\alpha: \mathbf{C}^{\times} \rightarrow \operatorname{Aut}(L \rightarrow V)$. If $\alpha, \beta$ are two such $\mathbf{C}^{\times}$actions, let $A_{k}, B_{k}$ be the infinitesimal generators on $H^{0}\left(V, L^{k}\right)$ and define $\langle\alpha, \beta\rangle$ by the equation

$$
\operatorname{Tr}\left(A_{k} B_{k}\right)-\frac{\operatorname{Tr}\left(A_{k}\right) \operatorname{Tr}\left(B_{k}\right)}{\operatorname{dim} H^{0}\left(V, L^{k}\right)}=\langle\alpha, \beta\rangle k^{n+2}+O\left(k^{n+1}\right)
$$

Next we construct the extremal $\mathbf{C}^{\times}$action $\chi$ as follows. Let $T \subseteq$ Aut $(L \rightarrow$ $V$ ) be a maximal torus. Define $\chi: \mathbf{C}^{\times} \rightarrow T$ by requiring

$$
F(\alpha)=\langle\chi, \alpha\rangle \text { for all } \alpha: \mathbf{C}^{\times} \rightarrow T .
$$

Now let $L \rightarrow X$ be an ample line bundle over a smooth projective variety and let $T \subseteq$ Aut $(L \rightarrow X)$ be a maximal torus. Let $\rho: \mathbf{C}^{\times} \rightarrow \operatorname{Aut}(\mathcal{L} \rightarrow \mathcal{X} \rightarrow \mathbf{C})$ be a test configuration. Let $\mathcal{X}^{\times}=\pi^{-1}\left(\mathbf{C}^{\times}\right)$and define a homomorphism $T \rightarrow \operatorname{Aut}\left(\mathcal{L}^{\times} \rightarrow \mathcal{X}^{\times}\right)$by

$$
t \cdot l_{\tau}=\rho(\tau) t \rho\left(\tau^{-1}\right) l_{\tau} \quad \text { for all } \quad l_{\tau} \in L_{\tau}
$$

We say that $\rho$ is compatible with $T$ if the action defined by (10.14) extends to an action $T \rightarrow \operatorname{Aut}(\mathcal{L} \rightarrow \mathcal{X})$. If $\rho$ is compatible with $T$, we let $\tilde{\chi}: \mathbf{C}^{\times} \rightarrow$ Aut $\left(L_{0} \rightarrow X_{0}\right)$ be the restriction of $\chi: \mathbf{C}^{\times} \rightarrow T \rightarrow \operatorname{Aut}(\mathcal{L} \rightarrow \mathcal{X})$ to the central fiber, and we let $\tilde{\rho}: \mathbf{C}^{\times} \rightarrow \operatorname{Aut}\left(L_{0} \rightarrow X_{0}\right)$ be the restriction of $\rho$ to the central fiber.

Definition 4. Let $(X, L)$ be a polarized variety and $T \subseteq$ Aut $(L \rightarrow X)$ a maximal torus. Let $\chi: \mathbf{C}^{\times} \rightarrow T$ be the extremal $\mathbf{C}^{\times}$action. We say that $(X, L)$ is $K$-stable relative to $T$ if for all test configurations $\rho$ compatible with $T$, we have

$$
F_{\tilde{\chi}}(\tilde{\rho}) \equiv F(\tilde{\rho})-\langle\tilde{\chi}, \tilde{\rho}\rangle \geq 0
$$

with equality if and only if the test configuration is a product.

It is then conjectured in [136] that a polarized variety $L \rightarrow X$ admits an extremal metric if and only if it is $K$-stable relative to a maximal torus.

\section{General $L$ : toric varieties}

In the case of general $L$, perhaps the greatest advances in the direction of sufficiency have taken place in the context of toric varieties, where the equations for the Kähler potential can be re-expressed in terms of its Legendre transform, namely the symplectic potential. They become then real equations, and the tools from convex analysis can be brought to bear. There have been many remarkable developments in this direction, even a perfunctory description of which is beyond the scope of this paper. We shall limit ourselves to a few words, mainly to provide references for further reading. 
11.1. Symplectic potentials. The basic properties of Kähler forms and their scalar curvatures on toric varieties have been worked out by Guillemin [67] and Abreu [1]. A brief summary is as follows. Let $L \rightarrow X$ be a positive toric line bundle over a toric variety. Then a dense orbit in $X$ of the $\left(\mathbf{C}^{\times}\right)^{n}$ action can be parametrized by $z=\left(z_{1}, \ldots, z_{n}\right)$, with $z_{i}=\xi_{i}+i \eta_{i}$. If $\omega$ is a Kähler form on the orbit which is invariant under the $\left(S^{1}\right)^{n}$ subgroup of $\left(\mathbf{C}^{\times}\right)^{n}$, then $\omega$ must be of the form

$$
\omega=\frac{i}{2} \frac{\partial^{2} \phi}{\partial \xi_{j} \partial \xi_{k}} d z^{j} \wedge d \bar{z}^{k}
$$

with $\phi$ a strictly convex function of the variables $\xi_{i}$ alone, $\phi=\phi(\xi)$. Associated to $\phi$ is its Legendre transform $u(x)$ defined by

$$
u(x)=\sup _{\zeta}(\langle x, \zeta\rangle-\phi(\zeta))=\langle x, \xi\rangle-\phi(\xi), \quad x=\frac{\partial \phi}{\partial \xi},
$$

which is a function on the polytope $P$ given by the image of the moment map

$$
\mu: z \rightarrow x=\frac{\partial \phi}{\partial \xi} .
$$

The polytope $P$ does not depend on the choice of Kähler form $\omega$ within $c_{1}(L)$. The function $u(x)$ is called the symplectic potential. It is strictly convex. The metric $\omega$ can be re-written in terms of the coordinates $(x, \eta)$ as $u_{i j} d x^{i} d x^{j}+u^{i j} d \eta^{i} d \eta^{j}$, where $u_{i j}$ is the Hessian metrix of $u_{i j}$, and $u^{i j}$ is its inverse. The scalar curvature becomes

$$
R(\omega)=-\frac{\partial^{2} u^{i j}}{\partial x^{i} \partial x^{j}} .
$$

The form $\omega$ extends to a smooth Kähler form on $X$ if and only if $u(x)$ satisfies

$$
u(x)-\frac{1}{2} \sum \delta_{k}(x) \log \delta_{k}(x) \in C^{\infty}(\bar{P})
$$

Here we have written $P$ as the intersection of half-spaces $\delta_{k}(x)>0$.

11.2. $K$-stability on toric varieties. The main concepts in $K$ stability can also be re-written completely explicitly in terms of the symplectic potential $u$ and the polytope $P$. In particular, test configurations correspond precisely to rational piecewise linear convex functions $f$ on $P$, and the $K$-energy and the Futaki invariant are given by the following functionals of $u[47]$,

$$
\begin{aligned}
F(f) & =-\mu \int_{P} f d x+\int_{\partial P} f d \sigma \\
K(u) & =-\int_{P} \log \left(\operatorname{det} u_{i j}\right) d x+F(u),
\end{aligned}
$$

where $d x$ is the Lebesgue measure on $P$ and $d \sigma$ is the measure on $\partial P$ defined by the requirement that $d \sigma \wedge d \delta_{k}= \pm d x$ on the face defined by $\delta_{k}(x)=0$. 
The problem reduces then to finding a smooth convex solution in $P$ for the following 4-th order equation, called Abreu's equation,

$$
-\frac{\partial^{2} u^{i j}}{\partial x^{i} \partial x^{j}}=\bar{R}
$$

subject to the boundary condition (11.5), assuming that the FutakiDonaldson invariant is strictly positive for all test configurations. This remains a challenging problem, but when $\operatorname{dim} X=2$, the following progresses have been made by Donaldson:

(a) It has been shown in [47] that $K$-stability implies that the $K$-energy is bounded from below;

(b) Interior a priori estimates for Abreu's equation have been obtained in $[48]$;

(c) A method of continuity has been developed in [52].

Generalized solutions, assuming the properness of the $K$-energy, are also studied in [167].

11.3. The $\boldsymbol{K}$-unstable case. In the $K$-unstable case, it has been shown by Szekelyhidi [138] that there is a maximally destabilizing configuration, in the class of functions which are in $L^{2}(P)$, continuous and convex on the union of $P$ with its codimension 1 faces, and whose boundary values are in $L^{1}(\partial P)$. It is not yet known, and perhaps not always the case that this configuration is piecewise linear. If it is, he also shows how this maximally destabilizing configuration would lead to a decomposition into semi-stable components analogous to the Harder-Narasimhan filtration for an unstable vector bundle. The Calabi functional is the analogue in this context of the Yang-Mills functional. In general, it is not known and not expected that this maximally destabilizing configuration should be piecewise linear. Thus it should be viewed as a limit of test configurations [138]. An attractive scenario for how to construct still a Harder-Narasimhan like filtration is also given in $[\mathbf{1 3 8}]$.

\section{Geodesics in the space $\mathcal{K}$ of Kähler potentials}

As we have seen in section $§ 6.2$, Donaldson's infinite-dimensional GIT theory provides yet another possible approach to the problem of constant scalar curvature metrics. In this approach, geodesic rays in the space $\mathcal{K}$ of Kähler potentials play the role of test configurations, and numerical invariants and stability conditions are to be constructed from the asymptotic behavior of the $K$-energy along geodesic rays.

The starting point for this approach is then the construction and regularity of geodesics. We have seen earlier that this can be viewed as an existence and regularity problem for a Dirichlet problem for a completely degenerate Monge-Ampère equation. There has been significant progress in this direction, some of which we describe below. 
12.1. The Dirichlet problem for the complex Monge-Ampère equation. Let $L \rightarrow X$ be a positive line bundle over a compact complex manifold $X$ of dimension $n$. Let $h_{0}$ be a metric on $L$, with $\omega_{0}=$ $-\frac{i}{2} \partial \bar{\partial} \log h_{0}>0$. Let $A=\left\{w \in \mathbf{C} ; e^{-T}<|w|<1\right\}$, and write $\Omega_{0}$ for $\omega_{0}$, viewed as a non-negative $(1,1)$-form on $X \times A$. We consider the following Dirichlet problem

$$
\left(\Omega_{0}+\frac{i}{2} \partial \bar{\partial} \Phi\right)^{n+1}=0 \text { on } X \times A,\left.\quad \Phi\right|_{X \times \partial A}=\Phi_{b},
$$

where $\Phi$ is continuous at $X \times \partial A$, and the boundary $\partial A$ and the boundary data are specified in the following two possible manners:

- Geodesic segments: Here another metric $h_{1}$ on $L$ with $\omega_{1}=-\frac{i}{2} \partial \bar{\partial}$ $\log h_{1}>0$ is prescribed, $T>0$ is finite, $\partial A \equiv\left\{w \in \mathbf{C} ;|w|=1\right.$ or $\left.|w|=e^{-T}\right\}$, and the boundary value $\Phi_{b}$ is given by

$$
\begin{array}{ll}
\Phi_{b}(z, w)=0 & \text { for }|w|=1 \\
\Phi_{b}(z, w)=\log \frac{h_{1}(z)}{h_{0}(z)} & \text { for }|w|=e^{-T} .
\end{array}
$$

- Geodesic rays: Here $T=\infty$, and $\partial A \equiv\{w \in \mathbf{C} ;|w|=1\}$, and the boundary value $\Phi_{b}$ is given by

$$
\Phi_{b}(z, w)=0 \quad \text { for }|w|=1 .
$$

12.2. Method of elliptic regularization and a priori estimates. The theory of a priori estimates for elliptic Monge-Ampère equations has been developed by Calabi [22], Yau [157], and Aubin [5] in the compact case, and by Caffarelli, Kohn, Nirenberg, and Spruck [18] in the case of domains in $\mathbf{C}^{n}$ with boundary. The geodesics problem requires an extension to the case of degenerate equations on complex manifolds with boundary. The following theorem was established in [115]. The main interest lies in its formulation, otherwise it is a generalization, requiring no new estimates, of results in Chen [27]. As can be seen from the sketch of the proof given below, it is a direct outgrowth of the estimates for Monge-Ampère equations obtained in $[\mathbf{1 8}, \mathbf{1 5 7}, \mathbf{6 2}, \mathbf{2 7}]$ :

THEOREM 22. Let $\bar{M}$ be a compact complex manifold of dimension $m$, with smooth boundary $\partial M$. Assume that $\bar{M}$ admits a Kähler form $\Omega$. Then the Dirichlet problem for the completely degenerate Monge-Ampère equation

$$
\left(\Omega+\frac{i}{2} \partial \bar{\partial} \Psi\right)^{m}=0 \text { on } M,\left.\quad \Psi\right|_{\partial M}=0
$$

for a function $\Psi$ which is $\Omega$-plurisubharmonic, admits a unique $C^{1,1}$ solution.

We sketch the proof of Theorem 22. Consider the elliptic regularization

$$
\left(\Omega+\frac{i}{2} \partial \bar{\partial} \Psi\right)^{m}=\epsilon \Omega^{m} \text { on } M,\left.\quad \Psi\right|_{\partial M}=0
$$


for a constant $\epsilon>0$ which we shall ultimately let tend to 0 . Since $\Omega$ is a Kähler form, and hence positive definite, the function $\underline{\Psi}=0$ is a subsolution of (12.5) for any $0<\epsilon \leq 1$. The elliptic regularization admits then a $C^{\infty}$ solution, and it suffices to establish $C^{2}$ estimates for the solution uniform in $\epsilon$ in order to obtain the theorem.

- The $C^{0}$ estimates follow as in the case of domains in $\mathbf{C}^{m}$ from the maximum principle [18], once a subsolution has been constructed;

- In the case of domains in $\mathbf{C}^{m}$, the estimates for the first-order derivatives can be reduced to the boundary, by differentiating the equation along any constant, global vector field, and applying the maximum principle. Since the solution is bounded by the subsolution and a harmonic function with same boundary condition, it follows that the boundary values of its gradient must be bounded [18]. This argument does not generalize to complex manifolds. However, the blow-up arguments of Chen [27] still apply.

- The differential inequalities of Yau [157] and Aubin [5] for the trace of the Hessian of the solution hold on general Kähler manifolds. Applying the maximum principle, they reduce the estimates for the second derivatives to the boundary. A difficult barrier argument gives next these boundary estimates, in the case of strongly pseudoconvex domains in $\mathbf{C}^{n}$ [18]. This barrier argument was subsequently extended by Guan [62] to the general case, assuming instead of strong pseudoconvexity the existence of a subsolution with given boundary values. Q.E.D.

We observe that the $C^{2}$ estimates give an upper bound for the eigenvalues of $\Omega+\frac{i}{2} \partial \bar{\partial} \Psi$. If the Monge-Ampère equation is elliptic, the determinant is bounded from below, and hence all the eigenvalues are bounded from both above and below. The $C^{3}$ identity of Calabi and Yau can then apply, reducing again the $C^{3}$ estimates to the boundary. An ingenious argument is then provided in [18] for the logarithmic modulus of continuity of the second derivatives, and hence the $C^{3}$ boundary estimates. Alternative approaches have also been provided by Evans [54] and Krylov [81].

In the degenerate case, the $C^{2}$ estimates do not imply bounds from below for the eigenvalues of the form $\Omega+\frac{i}{2} \partial \bar{\partial} \Psi$, and we cannot go further and obtain estimates for higher order derivatives. This is consistent with the optimal $C^{1,1}$ regularity of known examples of solutions of degenerate MongeAmpère equations $[\mathbf{6 0}, \mathbf{8 2}]$. A partial regularity theory for completely degenerate complex Monge-Ampère equations has been put forth in [34].

We return now to the problem proper of geodesics in the space $\mathcal{K}$ of Kähler potentials for a positive line bundle $L \rightarrow X$. We consider first the case of geodesic segments, when $M=X \times A$ with $A$ an annulus, the case of geodesic rays being somewhat different and treated separately later. The following simple, but key lemma will allow to reduce the original 
equation (12.1) with degenerate background form $\Omega_{0}$ to the situation treated in Theorem 22:

LEMma 14. There exists a function $\Phi \in C^{\infty}(X \times \bar{A})$ which is a subsolution of the equation (12.1), in the sense that

$$
\Omega_{0}+\frac{i}{2} \partial \bar{\partial} \underline{\Phi}>0,\left.\quad \underline{\Phi}\right|_{X \times \partial A}=\Phi_{b} .
$$

ProOF. Let $\phi(z, t)=t \log \frac{h_{0}(z)}{h_{1}(z)}$, and $\underline{\tilde{\Phi}}(z, w)=\phi(z, t), t=\log |w|$. Then clearly $\left.\underline{\tilde{\Phi}}\right|_{X \times \partial A}=\Phi_{b}$, and we have

$$
\Omega_{0}+\frac{i}{2} \partial \bar{\partial} \underline{\tilde{\Phi}}(z, w)=\left(\begin{array}{cc}
(1-t) \omega_{0}+t \omega_{1} & \frac{i}{4 w} \partial_{\bar{z}} \log \frac{h_{0}(z)}{h_{1}(z)} \\
-\frac{i}{4 \bar{w}} \partial_{z} \log \frac{h_{0}(z)}{h_{1}(z)} & 0
\end{array}\right)
$$

The upper left entry is uniformly strictly plurisubharmonic on $X$. Thus, setting $\underline{\Phi}(z, w)=\underline{\Phi}(z, w)+f(w)$, where $f(w)$ is the solution of the Dirichlet problem $\Delta f=C,\left.f\right|_{\partial A}=0$ for some constant $C>0$ large enough, we obtain the desired subsolution. Q.E.D.

Let now $\Omega=\Omega_{0}+\frac{i}{2} \partial \bar{\partial} \underline{\Phi}, \Psi=\Phi-\underline{\Phi}$. The equation (12.1) for a function $\Phi$ which is $\Omega_{0}$-plurisubharmonic, is then equivalent to the equation in (12.4) on $\bar{M}=X \times \bar{A}$, for a function $\Psi$ which is $\Omega$-plurisubharmonic. Theorem 22 gives then the following existence and $C^{1,1}$ regularity for geodesic segments which was proved by X.X. Chen [27], and which led to the more general formulation provided in Theorem 22:

Theorem 23. [27] Let $L \rightarrow X$ be a positive line bundle over a compact complex manifold, and $h_{0}, h_{1} C^{\infty}$ metrics on $L$ with positive curvatures $\omega_{0}, \omega_{1}$. Then the Dirichlet problem (12.1) admits a unique $C^{1,1}$ solution. The solution is $C^{1}$ invariant, and defines a $C^{1,1}$ geodesic joining $h_{0}$ to $h_{1}$ in the space $\mathcal{K}_{k}$ of Kähler potentials.

Next, we discuss the construction of geodesic rays. Recall that they are the analogues for $\mathcal{K}$ of the one-parameter subgroups in $\mathcal{K}_{k}$. Since oneparameter subgroups are essentially the same as test configurations, the natural question which arises is whether one can associate a geodesic ray to each test configuration.

In [4], Arezzo and Tian showed that, given a test configuration $\mathcal{T}$ for $L \rightarrow$ $X$ with smooth central fiber $X_{0}$, then one can use the Cauchy-Kowalevska theorem to find families of local analytic solutions to the geodesic equations near infinity. The geodesic rays obtained in this manner are real-analytic, but their origins cannot be prescribed. The condition that the central fiber be smooth is also a severe restriction. In [115], the following was established:

TheOREM 24. Let $L \rightarrow X$ be a positive line bundle on a compact complex manifold, and let $\mathcal{T}: \mathcal{L} \rightarrow \mathcal{X} \rightarrow \mathbf{C}$ be a test configuration for $L \rightarrow X$ in the sense of Definition 2. Let $h_{0}$ be any metric on $L$ with $\omega_{0}=-\frac{i}{2} \partial \bar{\partial} \log h_{0}>0$, 
and consider the Dirichlet problem (12.1) in the geodesic ray case, that is, when $A=\{w \in \mathbf{C} ; 0<|w| \leq 1\}=D^{\times}$, and $\partial A \equiv\{w \in \mathbf{C} ;|w|=1\}$.

Let $p: \tilde{\mathcal{X}} \rightarrow \mathcal{X} \rightarrow \mathbf{C}$ be any smooth, $S^{1}$ equivariant resolution of $\mathcal{X}$. Then the Dirichlet problem admits a solution $\Phi: X \times D^{\times} \rightarrow \mathbf{R}$, with $\Omega_{0}+$ $\frac{i}{2} \partial \bar{\partial} \Phi$ the restriction to $p^{-1}\left(\mathcal{X}_{\left.\right|_{D^{\times}}}\right)$of a non-negative $(1,1)$ current $\Omega+\frac{i}{2} \partial \bar{\partial} \Psi$ on $\tilde{\mathcal{X}}_{D} \equiv p^{-1}\left(\mathcal{X}_{\left.\right|_{D}}\right)$ satisfying

$$
\left(\Omega+\frac{i}{2} \partial \bar{\partial} \Psi\right)^{n+1}=0 \quad \text { on } \quad \tilde{\mathcal{X}}_{\left.\right|_{D}} .
$$

Here $\Omega$ is a smooth Kähler metric on $\tilde{\mathcal{X}}_{\left.\right|_{D}}$, and $\Psi$ is a $C^{1,1}$ function.

A key step in the proof of this theorem is the construction of the Kähler form $\Omega$ on $\tilde{\mathcal{X}}_{\left.\right|_{D}}$, the remaining part following readily from Theorem 22 . This is accomplished by constructing a line bundle $\mathcal{M} \rightarrow \tilde{\mathcal{X}}$ with the properties that

(a) $p^{*} \mathcal{L}^{m} \otimes \mathcal{M} \rightarrow \tilde{\mathcal{X}}$ is a positive line bundle;

(b) $\left.\mathcal{M}\right|_{\tilde{\mathcal{X}}^{\times}}$is trivial, in the sense that $\mathcal{M}$ admits a meromorphic section which is holomorphic and nowhere vanishing on $\tilde{\mathcal{X}}^{\times}$.

The desired Kähler form can then be taken as the curvature of $p^{*} \mathcal{L}^{m} \otimes$ $\mathcal{M} \rightarrow \tilde{\mathcal{X}}$.

12.3. Geodesics in $\mathcal{K}$ and geodesics in $\mathcal{K}_{k}$. An important question in the problem of constant scalar curvature metrics is to determine in what precise sense Donaldson's infinite-dimensional GIT is the limit of GIT. In this context, it would be particularly valuable to realize geodesics in $\mathcal{K}$ as limits of geodesics in $\mathcal{K}_{k}$. This can be viewed also as the natural next step in Yau's general strategy of approximations by algebraic-geometric objects: the Tian-Yau-Zelditch theorem says that $\mathcal{K}$ is the "pointwise" limit of $\mathcal{K}_{k}$, and the natural next step is to understand the external geometry of $\mathcal{K}_{k}$ as $k \rightarrow \infty$. If geodesics in $\mathcal{K}$ can be approximated by geodesics in $\mathcal{K}_{k}$, as is desirable from the point of view of GIT, this would mean that the subspaces $\mathcal{K}_{k}$ satisfy a remarkable property, namely that they become asymptotically geodesically flat as $k \rightarrow \infty$. In this section, we describe in what precise sense the answer to this question is indeed affirmative $[\mathbf{1 1 3}, \mathbf{1 1 4}]$.

12.3.1. An Ansatz for geodesic approximations. Our approach is based on the following general Ansatz $[\mathbf{1 1 2}, \mathbf{1 1 3}, \mathbf{1 1 5}]$. Let $L \rightarrow X$ be a positive line bundle over a compact complex manifold $X$ of dimension $n$, and let $h_{0}$ be a metric on $L$ with positive curvature $\omega_{0}=-\frac{i}{2} \partial \bar{\partial} \log h_{0}$. Let $\underline{s}=\left\{s_{\alpha}\right\}_{\alpha=0}^{N_{k}}$ be an orthonormal basis for $H^{0}\left(X, L^{k}\right)$ with respect to the metric $h_{0}$ and the volume form $\omega_{0}^{n}$. For each $k>>1$, let $\lambda_{\alpha}^{(k)}$ be a sequence of real numbers, $0 \leq \alpha \leq N_{k}=\operatorname{dim} H^{0}\left(X, L^{k}\right)$. Set

$$
\Phi_{k}(z, w)=\frac{1}{k} \log \sum_{\alpha=0}^{N_{k}}|w|^{2 \lambda_{\alpha}^{(k)}}\left|s_{\alpha}(z)\right|^{2} h_{0}^{k}-n \frac{\log k}{k} .
$$


The function $\Phi_{k}(z, w)$ is manifestly $\Omega_{0}$-plurisubharmonic on $X \times D^{\times}$, where $D^{\times}=\{w \in \mathbf{C} ; 0<|w|<1\}$, and $\Omega_{0}$ is the form $\omega_{0}$, viewed as a non-negative form on $X \times D^{\times}$. Let

$$
\Phi(z, w)=\lim _{\ell \rightarrow \infty}\left[\sup _{k \geq \ell} \Phi_{k}(z, w)\right]^{*},
$$

where $u^{*}(z) \equiv \lim _{\epsilon \rightarrow 0}\left(\sup _{|z-\zeta|<\epsilon} u(\zeta)\right)$ denotes the upper semi-continuous envelope of a function $u(z)$. Then $\Phi(z, w)$ is a $\Omega_{0}$-plurisubharmonic function on $X \times D^{\times}$, and $\Phi_{k}(z, w)=\phi_{k}(z, \log |w|)-n \frac{\log k}{k}$, where

$$
\phi_{k}(z, t)=\frac{1}{k} \log \sum_{\alpha=0}^{N_{k}} e^{2 \lambda_{\alpha}^{(k)} t}\left|s_{\alpha}(z)\right|^{2} h_{0}^{k}
$$

is a geodesic in the space $\mathcal{K}_{k}$ of Bergman potentials. Geometrically, the expression (12.9) has another nice motivation. Let $\pi_{*}\left(L^{k}\right)$ be the direct image of $L^{k}$ over $A_{T}$, that is, the vector bundle over $A_{T}$ whose fiber at each $w \in A_{T}$ is the vector space $H^{0}\left(X, L^{k}\right)$. Holomorphically, the bundle is trivial. However, the choice of a metric $h_{0}$ on $L$ equips the fiber of $\pi_{*}\left(L^{k}\right)$ at, say, $w=1$ with the corresponding $L^{2}$ metric, and then, by rotation $w \rightarrow e^{i \theta} w$, to the fibers along $|w|=1$. Then, at each $w \in A_{T}$, the expression

$$
\left\langle s_{\alpha}, s_{\beta}\right\rangle=|w|^{2 \lambda_{\alpha}^{(k)}} \delta_{\alpha \bar{\beta}}
$$

in (12.9) defines a metric which restricts on the boundary of $A_{T}$ to the $L^{2}$ metric induced by $h_{0}$, and is flat. The flatness is a consequence of the fact that the bundle $\pi_{*}\left(L^{k}\right)$ admits locally an orthonormal basis of holomorphic sections, namely $w^{-\lambda_{\alpha}^{(k)}} s_{\alpha}$. Returning to our original problem, the question is then under what circumstances does $\Phi$ satisfy the degenerate MongeAmpère equation. The following theorem can be extracted from $[\mathbf{1 1 3}, \mathbf{1 1 4}]$ and provides an answer to this question:

TheOrem 25. Let the set-up be as described above. Let $A_{T}=\{w \in$ $\left.\mathbf{C} ; e^{-T}<|w|<1\right\}$, where $T$ can be both finite or infinite. If the following two conditions are satisfied,

(a) There exists a constant $C>0$, independent of both $k$ and $\alpha$, so that

$$
\left|\lambda_{\alpha}^{(k)}\right| \leq C k
$$

(b) There exists a constant $C>0$ independent of $k$ so that

$$
\iint_{X \times A_{T}} \Omega_{k}^{n+1} \leq C k^{-1}
$$

then $\Phi(z, w)$ is continuous near $|w|=1$, and we have, in the sense of pluripotential theory,

$$
\left(\Omega_{0}+\frac{i}{2} \partial \bar{\partial} \Phi\right)^{n+1}=0 \text { on } X \times\left. A_{T} \quad \Phi\right|_{|w|=1}=0 .
$$


Here the notion of $\left(\Omega_{0}+\frac{i}{2} \partial \bar{\partial} \Phi\right)^{n+1}$ in the sense of pluripotential theory, for $\Phi$ a $\Omega_{0}$-plurisubharmonic function, can be defined as follows. It suffices to define it locally, so we consider the case of a plurisubharmonic $u$ function on $\mathbf{C}^{n}$. Let $C$ be any non-negative $(1,1)$ closed current. Since the coefficients of $C$ are then non-negative measures, we may define

$$
(i \partial \bar{\partial} u) \wedge C=i \partial \bar{\partial}(u C) .
$$

Applying this to $C=i \partial \bar{\partial} u$, and iterating, we obtain a definition of $(i \partial \bar{\partial} u)^{n}$ as an $(n, n)$-form with a positive measure as coefficient.

Some steps in the proof of Theorem 25 are as follows. The condition (a) guarantees some a priori estimates for $\Phi_{k}(z, w)$, including a crucial uniform bound for the normal derivative of $\Phi_{k}(z, w)$ at the component $|w|=1$ of the boundary of $A_{T}$, which will guarantee that $\Phi$ has the desired boundary value. The condition (b) suggests that a reasonable limit of $\Phi_{k}(z, w)$ should have mass 0 , and thus satisfy the degenerate Monge-Ampère equation. In the foundational work $[\mathbf{1 0}, \mathbf{1 1}]$ of Bedford and Taylor, it is shown that this is indeed the case, if the $\Phi_{k}(z, w)$ converge either uniformly or monotonically. This is not the situation in the setting of Theorem 25, but a suitable extension of the Bedford-Taylor pluripotential theory can be established [113], which does give the desired convergence, when $T$ is finite and we have a standard Dirichlet problem for a domain with smooth codimension 1 boundary. When $T=\infty$, the argument has to be supplemented by a careful limiting process, first with $T$ finite, and then letting $T$ tend to $\infty$ [114].

Clearly, the assumption (b) can be weakened to $\iint_{X \times A_{T}} \Omega_{k}^{n+1} \rightarrow 0$, in which case the conclusion of the theorem would be that there exists a subsequence $\Phi_{k_{j}}(z, w)$, whose corresponding limit $\Phi(z, w)$ in the sense of (12.10) satisfies the equations (12.15).

Other extensions of the Bedford-Taylor theory to the manifold setting can be found in $[\mathbf{6 6}]$ and $[\mathbf{1 5}]$.

12.3.2. Construction of geodesic segments. We apply Theorem 25 in the context of geodesic segments. In this case, we are given a second metric $h_{1}$ on $L$ with positive curvature $\omega_{1}=-\frac{i}{2} \partial \bar{\partial} \log h_{1}$, and the problem is to construct the geodesic joining $h_{0}$ to $h_{1}$ (whose existence has been proved by the Theorem 23 of X.X. Chen, using a priori estimates and elliptic regularization). In this case, let $\underline{s}^{(1)}=\left\{s_{\alpha}^{(1)}(z)\right\}_{\alpha=0}^{N_{k}}$ be a basis for $H^{0}\left(X, L^{k}\right)$, orthonormal this time with respect to the metric $h_{1}$ and the volume form $\omega_{1}^{n}$. Without loss of generality, we may assume that the change of bases from $\underline{s}$ to $\underline{s}^{(1)}$ is given by a diagonal matrix,

$$
s_{\alpha}^{(1)}=e^{\lambda_{\alpha}^{(k)}} s_{\alpha} .
$$

Let $\Phi_{k}(z, w)$ be defined as in (12.10), with this choice of weights $\lambda_{\alpha}^{(k)}$. Then it is shown in [113] that the two conditions (a) and (b) of Theorem 25 are satisfied. It may be instructive to see how to verify condition (b), assuming condition (a), since we have already at our disposal all the tools needed to 
estimate the Monge-Ampère masses in this context. By (5.18), this reduces to computing $\dot{F}_{\omega}^{0}$ at the two end points $t=0$ and $t=1$, and since $\phi_{k}(z, t)$ is a path inside $\mathcal{K}_{k}$, the formulas for $\dot{F}$ in section $\S 5.1$ apply. Thus we have

$$
\iint_{X \times A_{T}} \Omega_{k}^{n+1}=\int_{X} \dot{\phi}_{k}(1) \omega_{\phi(1)}^{n}-\int_{X} \dot{\phi}_{k}(0) \omega_{\phi(0)}^{n}
$$

and hence, more explicitly,

$$
\begin{aligned}
& \frac{2}{k^{n+1}} \int_{X} \sum_{\alpha=0}^{N_{k}} \lambda_{\alpha}^{(k)}\left|s_{\alpha}^{(1)}(z)\right|^{2} h_{1}(k)^{k} \omega_{1}(k)^{n} \\
& \quad-\frac{2}{k^{n+1}} \int_{X} \sum_{\alpha=0}^{N_{k}} \lambda_{\alpha}^{(k)}\left|s_{\alpha}(z)\right|^{2} h_{0}(k)^{k} \omega_{0}(k)^{n} .
\end{aligned}
$$

Applying the Tian-Yau-Zelditch theorem, we obtain easily the following asymptotics,

$$
\begin{aligned}
& \frac{2}{k^{n+1}} \int_{X} \sum_{\alpha=0}^{N_{k}} \lambda_{\alpha}^{(k)}\left|s_{\alpha}(z)\right|^{2} h_{1}(k)^{k} \omega_{1}(k)^{n} \\
& =\frac{2}{k^{n+1}} \sum_{\alpha=0}^{N_{k}} \lambda_{\alpha}^{(k)}+O\left(\frac{1}{k^{n+2}}\right) \cdot N_{k} \cdot \max _{\alpha}\left|\lambda_{\alpha}^{(k)}\right|
\end{aligned}
$$

as well as an analogous expression with $h_{1} \leftrightarrow h_{0}$. The leading term in this expression cancels out between the contributions from $h_{1}$ and from $h_{0}$, and the second term is $O\left(k^{-1}\right)$ in view of condition (a). Thus condition (b) is satisfied. Theorem 25 implies then that the corresponding $\Phi(z, w)$ defines then a solution of the equation (12.15). Furthermore, the roles of $h_{0}$ and $h_{1}$ are clearly reversible, and it follows that $\Phi(z, w)$ satisfies the desired boundary condition also when $|w|=e^{-1}$, and is actually a solution of the Dirichlet problem (12.1). By uniqueness, it must coincide with the solution provided by Theorem 23. Thus we have the following theorem:

TheOREm 26. Let $L \rightarrow X$ be a positive line bundle over a compact complex manifold $X$. Let $h_{0}, h_{1}$ be two metrics on $L$ with positive curvatures $\omega_{i}=-\frac{i}{2} \partial \bar{\partial} \log h_{i}$. Let $\underline{s}, \underline{s}^{(1)}$ be two bases of $H^{0}\left(X, L^{k}\right)$, orthonormal with respect to the metrics and volume forms $h_{0}, \omega_{0}$ and $h_{1}, \omega_{1}$ respectively. Assume without loss of generality that the matrix of change of bases is diagonal, with eigenvalues $\lambda_{\alpha}^{(k)}$. Then the $C^{1,1}$ geodesic joining $h_{0}$ and $h_{1}$ can be obtained by the construction (12.10).

12.3.3. Construction of geodesic rays. We turn next to the construction of geodesic rays. Let $L \rightarrow X$ be a positive line bundle over a compact complex manifold as before, and let $\mathcal{T}: \mathcal{L} \rightarrow \mathcal{X} \rightarrow \mathbf{C}$ be a test configuration, in the sense of Definition 2. Let $A_{k}$ be the traceless endomorphism of $H^{0}\left(X_{0}, L_{0}^{k}\right)$, as defined in 6.45 and subsequent line, and let $\lambda_{\alpha}^{(k)}$ be its eigenvalues. Then $[\mathbf{1 1 4}]$ 
THEOREM 27. With this set-up, and this choice of weights $\lambda_{\alpha}^{(k)}$, the expressions (12.9) and (12.10) produce a generalized geodesic ray starting at $h_{0}$, i.e., $\Phi(z, w)$ is continuous and equal to 0 at $|w|=1$, and we have, in the sense of pluripotential theory,

$$
\left(\Omega_{0}+\frac{i}{2} \partial \bar{\partial} \Phi\right)^{n+1}=0 \quad \text { on } \quad X \times\{w \in \mathbf{C} ; 0<|w|<1\} .
$$

The geodesic is non-constant when the test configuration $\mathcal{T}$ is non-trivial.

To establish the theorem, we apply Theorem 25 and verify conditions (a) and (b). Once again, in this exposition, we assume condition (a), and concentrate on condition (b). Again, by (12.18), we can write

$$
\iint_{X \times A_{\infty}} \Omega_{k}^{n+1}=\lim _{T \rightarrow \infty} \int_{X} \dot{\phi}(T) \omega_{k}(T)^{n}-\int_{X} \dot{\phi}(0) \omega_{k}(0)^{n}
$$

We expand the second term on the right hand side, using the Tian-YauZelditch theorem as before. In view of (12.20) and the fact that the matrix $A_{k}$ is traceless, this term is $O\left(k^{-1}\right)$. By Lemma 6, we have

$$
\lim _{T \rightarrow \infty} \int_{X} \dot{\phi}(T) \omega_{k}(T)^{n}=\frac{1}{k} F
$$

where $F$ is the Donaldson-Futaki invariant. In particular, this shows that this term is also $O\left(k^{-1}\right)$, establishing condition (b). Thus Theorem 27 follows from Theorem 25.

The construction of Theorem 25 is arguably canonical, and thus, given a test configuration $\mathcal{T}$, we have a canonical way of associating to any point $h_{0} \in \mathcal{K}$ a generalized vector, namely the initial velocity vector at $h_{0}$ of the geodesic we have just constructed. In this sense, a test configuration defines a generalized vector field on the space $\mathcal{K}$ of Kähler potentials. It would be very valuable to be able to write this vector field down more explicitly in terms of data from the test configuration $\mathcal{T}$. Indeed, it would provide a valuable model for how to relate behavior at $\infty$ to behavior well inside $\mathcal{K}$, a ubiquitous underlying theme in the problem of stability and constant scalar curvature metrics.

12.3.4. Variations on the Ansatz and rates of convergence. Theorems 26 and 27 show that geodesics in $\mathcal{K}$ can be approximated by geodesics in $\mathcal{K}_{k}$. It is of great interest to understand how good such approximations can be, and in particular, to analyze more precisely the approximation given in (12.10). Replacing the potentials $\Phi_{k}$ in (12.10) with potentials $\tilde{\Phi}_{k}$ associated instead to the bundle $L^{k} \otimes K_{X}$, Berndtsson [12] has obtained another version of (12.10) with $C^{0}$ convergence and with precise error bounds $O\left(k^{-1} \log k\right)$. If we stay instead with $\Phi_{k}$, the sharpest results to date have been provided by Song and Zelditch $[\mathbf{1 3 2}, \mathbf{1 3 3}]$, in the context of toric varieties:

TheOREm 28. Let $L \rightarrow X$ be an ample toric line bundle over a compact toric variety. Let $h_{0}, h_{1}$ be toric Hermitian metrics on $L$ with positive 
curvatures $\omega_{0}, \omega_{1}$. Then the approximation $\Phi_{k}$ described in (12.9) converges in $C^{2}(X)$ to the geodesic joining $h_{0}$ and $h_{1}$.

We note that, for toric varieties, the geodesic equation becomes a linear equation for the Legendre transform of the potentials $[63,132]$, so that the geodesic joining $h_{0}$ and $h_{1}$ is known to be smooth if $h_{0}$ and $h_{1}$ are smooth.

A similar sharp analysis of the construction of geodesic rays associated to a test configuration by Theorem 27 has appeared very recently in $[\mathbf{1 3 4}]$. In particular, it is shown there that the regularity $C^{1,1}$ for such rays is optimal. Some remarkable and potentially far-reaching relations between the Ansatz (12.9) for complex Monge-Ampère equations and ideas from the theory of large deviations are also brought to light in this paper. Other unexpected connections between approximations of the form (12.9) and classical topics such as Bernstein polynomials and Dedekind-Riemann sums over lattice points in polytopes can be found in $[\mathbf{1 6 4}]$.

\section{References}

[1] Abreu, M., "Kähler geometry of toric varieties and extremal metrics", International J. Math. 9 (1998) 641-651.

[2] Apostolov, V., D. Calderbank, P. Gauduchon, and C. W. Tonnesen-Friedman, "Hamiltonian 2-forms in Kahler geometry, III Extremal metrics and stability", arXiv:math/0511118 [math.DG], to appear in Inv. Math.

[3] Arezzo, C. and F. Pacard, "Blowing up and desingularizing constant scalar curvature Kähler manifolds", Acta Math. 196 (2006), no. 2, 179-228.

[4] Arezzo, C. and G. Tian, "Infinite geodesic rays in the space of Kähler potentials", Ann. Sc. Norm. Sup. Pisa (5) 2 (2003) 617-630.

[5] Aubin, T., "Equations du type Monge-Ampère sur les varietes kähleriennes compactes", C.R. Acad. Sci. Paris 283 (1976) 119-121.

[6] Bando, S., "On three-dimensional compact Kähler manifolds of nonnegative bisectional curvature", J. Differential Geom. 19 (1984) 284-297.

[7] Bando, S., "The $K$-energy map, almost Einstein Kähler metrics and an inequality of Miyaoka-Yau type", Tohoku Math. J. 39 (1987) 231-235.

[8] Bando, S. and T. Mabuchi, "Uniqueness of Einstein Kähler metrics modulo connected group actions", Algebraic Geometry, Sendai, 1985, 11-40, Adv. Stud. Pure Math. 10, North-Holland, Amsterdam, 1987

[9] Batyrev, V. and E. Selivanova, "Einstein-Kähler metrics on symmetric toric Fano manifolds", J. Reine Angew. Math. 512 (1999), 225-236.

[10] Bedford, E. and B.A. Taylor, "The Dirichlet problem for a complex Monge-Ampère equation", Invent. Math. 37 (1976) 1-44.

[11] Bedford, E. and B.A. Taylor, "A new capacity for plurisubharmonic functions", Acta Math. 149 (1982) 1-40.

[12] Berndtsson, B., "Curvature of holomorphic bundles associated to holomorphic fibrations", arXiv: math.CV/0511225.

[13] Biquard, O., Séminaire Nicolas Bourbaki, Vol. 2004-2005, Asterisque, No. 307 (2006) Expose No. 938, 1-31.

[14] Blocki, Z., "The complex Monge-Ampère operator and pluripotential theory", lecture notes available from the author's website, http://www.im.uj.edu.pl/ blocki/publ

[15] Blocki, Z. and S. Kolodziej, "On regularization of plurisubharmonic functions on manifolds", Proc. Amer. Math. Soc. 135 (2007) no. 7, 2089-2093. 
[16] Bourguignon, J.P., P. Li, and S.T. Yau, "Upper bound for the first eigenvalue of algebraic submanifolds", Comment. Math. Helv. 69 (1994) 199-207.

[17] Boutet de Monvel, L. and J. Sjöstrand, "Sur la singularité des noyaux de Bergman et de Szegö", Asterisque 34-35 (1976) 123-164.

[18] Caffarelli, L., J.J. Kohn, L. Nirenberg, and J. Spruck, "The Dirichlet problem for non-linear second-order elliptic equations II. Complex Monge-Ampère equations and uniformly elliptic equations", Commun. Pure Appl. Math. XXXVIII (1985) 209-252.

[19] Caffarelli, L., L. Nirenberg, and J. Spruck, "The Dirichlet problem for non-linear second-order elliptic equations I. Monge-Ampère equations", Commun. Pure Appl. Math. XXXVII (1984) 369-402.

[20] Calabi, E., "Extremal Kähler metrics", Seminar on Differential Geometry, Ann. Math. Studies 16 (1982) 259-290.

[21] Calabi, E. and X.X. Chen, "The space of Kähler metrics II", J. Differential Geom. 61 (2002) 173-193.

[22] Cao, H.D., "Deformation of Kähler metrics to Kähler-Einstein metrics on compact Kähler manifolds", Invent. Math. 81 (1985) 359-372.

[23] Catlin, D., "The Bergman kernel and a theorem of Tian", Analysis and geometry in several complex variables, Katata 1999, Trends in Math. (1999) 1-23, Birkhäuser.

[24] Cegrell, U., "Capacities in complex analysis", Aspects of Math. E14, Vieweg, 1988

[25] Cheeger, J., "Finiteness theorems for Riemannian manifolds", Amer. J. Math. 92 (1970) 61-74.

[26] Chen, X.X., "Calabi flow in Riemann surfaces revisited: a new point of view", Internat. Math. Res. Notices (2001) no. 6, 275-297.

[27] Chen, X.X., "The space of Kähler metrics", J. Differential Geom. 56 (2000) 189-234.

[28] Chen, X.X., "Space of Kähler metrics III: on the lower bound of the Calabi energy and geodesic distance", arXiv: math.DG/0606228.

[29] Chen, X.X. and W. He, "On the Calabi flow", arXiv; 0603523 [math.DG].

[30] Chen, X.X. and H. Li, "Stability of Kähler-Ricci flow", arXiv:0801.3086.

[31] Chen, X.X. and Y. Tang, "Test configurations and geodesic rays", arXiv:07074149 [math.DG].

[32] Chen, X.X. and G. Tian, "Ricci flow on Kähler-Einstein surfaces", Invent. Math. 147 (2002) 487-544.

[33] Chen, X.X. and G. Tian, "Ricci flow on Kähler-Einstein manifolds", Duke Math. J. 131 (2006) 17-73.

[34] Chen, X.X. and G. Tian, "Geometric of Kähler metrics and foliations of holomorphic disks", arXiv : math.DG/0409433.

[35] Chow, B., "The Ricci flow on the 2-sphere", J. Differential Geom. 33 (1991) 325-334.

[36] Chow, B., Chu, S.C., Glickenstein, D., Guenther, C., Isenberg, J., Ivey, T., Knopf, D., Lu, P., Luo, F., Ni, L., "The Ricci flow: techniques and applications. Part I. Geometric aspects" Mathematical Surveys and Monographs, 135 Amer. Math. Soc., Providence, RI, 2007.

[37] Chow, B. and D. Knopf, "The Ricci flow: an introduction", Mathematical Surveys and Monographs, Vol. 110, Amer. Math. Soc., Providence, Rhode Island, 2004.

[38] Chrusciel, P., "Semi-global existence and convergence of the Robinson-Trautman (2-dimensional Calabi) equation", Commun. Math. Phys. 137 (1991) 289-313.

[39] Deligne, P., "Le determinant de la cohomologie", Contemporary Math. 67 (1987) 93-177.

[40] Demailly, J.P. and J. Kollár, "Semi-continuity of complex singularity exponents and Kähler-Einstein metrics on Fano orbifolds", Ann. Sci. Ecole Norm. Sup. 34 (2001) $525-556$.

[41] Ding, W.Y. and G. Tian, "Kähler-Einstein metrics and the generalized Futaki invariant", Invent. Math. 110 (1992) 315-335. 
[42] Dinh, T.C., Nguyen, V.A., and Sibony, N., "Exponential estimates for plurisubharmonic functions and stochastic dynamics", arXiv:0801.1983.

[43] Donaldson, S., "Infinite deteminants, stable bundles, and curvature", Duke Math. J. 54 (1987) 231-247.

[44] Donaldson, S., "Symmetric spaces, Kähler geometry, and Hamiltonian dynamics", Amer. Math. Soc. Transl. 196 (1999) 13-33.

[45] Donaldson, S., "Scalar curvature and projective imbeddings I", J. Differential Geometry 59 (2001) 479-522.

[46] Donaldson, S.K., "Holomorphic disks and the complex Monge-Ampère equation", J. Symplectic Geometry 1 (2002) no. 2, 171-196.

[47] Donaldson, S.K., "Scalar curvature and stability of toric varieties", J. Differential Geom. 59 (2002) 289-349.

[48] Donaldson, S.K., "Interior estimates for solutions of Abreu's equation", Collect. Math. 56 (2005), no. 2, 103-142.

[49] Donaldson, S.K., "Scalar curvature and projective imbeddings II", Q. J. Math. 56 (2005), no. 3, 345-356.

[50] Donaldson, S.K., "Lower bounds on the Calabi functional", J. Differential Geom. 70 (2005) 453-472.

[51] Donaldson, S.K., "Some numerical results in complex differential geometry", arXiv: math.DG/0512625.

[52] Donaldson, S.K., "Extremal metrics on toric surfaces, I", ArXiv:math/0612120.

[53] Donaldson, S.K., "A note on the $\alpha$-invariant of the Mukai-Umemura 3-fold", arXiv:0711.4357.

[54] Evans, L.C., "Classical solutions of fully non-linear, convex, second order elliptic equations", Comm. Pure Appl. Math. 25 (1982) 333-363.

[55] Fefferman, C., "The Bergman kernel and biholomorphic mappings of pseudoconvex domains", Inventiones Math. 24 (1974) 1-66.

[56] Fine J. and Ross, J., "A note on the positivity of the CM line bundle" IMRN, vol. 2006, Article ID 95875, 14 pages, 2006. math.AG/0605302.

[57] Futaki, A., "An obstruction to the existence of Einstein-Kähler metrics", Invent. Math. 73 (1983) 437-443.

[58] Futaki, A., "On a character of the automorphism group of a compact complex manifold", Invent. Math. 87 (1987) 655-660.

[59] Futaki, A., "Kähler-Einstein metrics and integral invariants", Lecture Notes in Mathematics 1314 (1988), Springer-Verlag, Berlin.

[60] Gamelin, T. and N. Sibony, "Subharmonicity for uniform algebras", J. Funct. Anal. 35 (1980), no. 1, 64-108.

[61] Gromov, M., "Structures metriques sur les varietes riemanniennes", Textes Math. 1, Cedric-Nathan, Paris, 1981.

[62] Guan, B., "The Dirichlet problem for the complex Monge-Ampère equation and regularity of the pluricomplex Green's function", Commun. Anal. Geom. 6 (1998) no. $4,687-703$.

[63] Guan, D., "On modified Mabuchi functional and Mabuchi moduli space of Kähler metrics on toric bundles", Math. Res. Lett. 6 (1999), no. 5-6, 547-555.

[64] Guan, D., "Extremal solitons and exponential $C^{\infty}$ convergence of the modified Calabi flow on certain $\mathbf{C P}^{1}$ bundles", Pacific J. Math. 233 (2007), no. 1, 91-124.

[65] Guan, P., N.S. Trudinger, and X.J. Wang, "On the Dirichlet problem for the degenerate Monge-Ampère equation", Acta Math. 82 (1999) no. 1, 87-104.

[66] Guedj, V. and A. Zeriahi, "Monge-Ampère operators on compact Kähler manifolds", arXiv:math.CV/0504234.

[67] Guillemin, V., "Kähler structures on toric varieties", J. Differential Geom. 40 (1994) 285-309. 
[68] Hamilton, R.S., "Three manifolds with positive Ricci curvature", J. Differential Geom. 17 (1982) 255-306.

[69] Hamilton, R.S., "Formation of singularities in the Ricci flow", Surveys in Differential Geom. II (1995) 7-136.

[70] Hamilton, R.S., "The Ricci flow on surfaces", Contemp. Math. 71 (1988) 237-261.

[71] Hamilton, R.S., "A compactness property for solutions of the Ricci flow", Amer. J. Math. 117 (1995) 545-572.

[72] Heier, G., "Convergence of the Kähler-Ricci flow and multiplier ideal sheaves on del Pezzo surfaces", arXiv: 0710.5725, math.AG.

[73] Hörmander, L., "An introduction to complex analysis in several variables", NorthHolland, 1984.

[74] Hou, Z. and Q. Li, "Energy functionals for the parabolic Monge-Ampère equation", arXiv:0704.1822.

[75] Kempf, G. and L. Ness, "The length of vectors in representation spaces", Algebraic Geometry (Proc. 1978 Summer Meeting, Copenhagen), Lecture Notes in Math. 732 (1979) 233-243, Springer-Verlag.

[76] Kim, J., C. LeBrun, and M. Pontecorvo, "Scalar flat Kähler surfaces of all genera", J. Reine Angew. Math 486 (1997) 69-95.

[77] Knudsen, F. and D. Mumford, "The projectivity of the moduli space of stable curves I. Preliminaries on det and Div", Math. Scand. 39 (1976) 19-55.

[78] Kohn, J.J., "Subellipticity of the $\bar{\partial}$-Neumann problem on weakly pseudo-convex domains: sufficient conditions", Acta Math. 142 (1979) 79-122.

[79] Kolodziej, S., "The complex Monge-Ampère equation", Acta Math. 180 (1998) 69117.

[80] Kolodziej, S., "The complex Monge-Ampère equation on compact Kähler manifolds", Indiana Univ. Math. J. 52 (2003) 667-686.

[81] Krylov, N.V., "Boundedly non-homogeneous elliptic and parabolic equations", Izvestia Akad. Nauk. SSSR 46 (1982) 487-523. English translation in Math. USSR Izv. 20 No. 3 (1983) 459-492.

[82] Lempert, L., "Solving the degenerate complex Monge-Ampère equation with one concentrated singularity", Math. Ann. 263 (1983) 515-532.

[83] LeBrun, C. and S. Simanca, "On Kähler surfaces of constant positive scalar curvature", J. Geom. Anal. 5 (1995) no. 1, 115-127.

[84] Li, H., "On the lower bound of the K energy and the F functional", arXiv:0609725.

[85] Lu, Z., "On the lower order terms of the asymptotic expansion of Tian-YauZelditch", Amer. J. Math. 122 (2000) 235-273.

[86] Lu, Z., "On the Futaki invariants of complete intersections", Duke Math. J. 100 (1999), no. 2, 359-372.

[87] Lu, Z., "On the lower bound estimates of sections of the canonical bundles over a Riemann surface", Internat. J. Math. 12 (2001), no. 8, 891-926.

[88] Lu, Z., " $K$ energy and $K$ stability on hypersurfaces.", Comm. Anal. Geom. 12 (2004), no. 3, 601-630.

[89] Luo, H., "Geometric criterion for Gieseker-Mumford stability of polarized manifolds", J. Differential Geom. 49 (1998) 577-599.

[90] Mabuchi, T., " $K$-energy maps integrating Futaki invariants", Tohoku Math. J. (2) 38 (1986), no. 4, 575-593.

[91] Mabuchi, T. "Some symplectic geometry on compact Kähler manifolds", Osaka J. Math. 24 (1987) 227-252.

[92] Mabuchi, T., "An energy-theoretic approach to the Hitchin-Kobayashi correspondence for manifolds. I", Invent. Math. 159 (2005), no. 2, 225-243.

[93] Mabuchi, T., "The Chow-stability and Hilbert-stability in Mumford's Geometric Invariant Theory", arXiv:math/0607590, [math.DG]. 
[94] Matsushima, Y., "Sur la structure du groupe d'homeomorphismes analytiques d'une certaine variete kählerienne", Nagoya. Math. J. 11 (1957) 145-150.

[95] Mok, N.M., "The uniformization theorem for compact Kähler manifolds of nonnegative holomorphic bisectional curvature", J. Differential Geom. 27 (1988) 179-214.

[96] Mori, S., "Projective manifolds with ample tangent bundles", Ann. of Math. 110 (1979) 593-606.

[97] Mumford, D., "Stability of projective varieties", L'Enseignement Mathematique 23 (1977) 39-110.

[98] Nadel, A., "Multiplier ideal sheaves and Kähler-Einstein metrics of positive scalar curvature", Ann. of Math. 132 (1990) 549-596.

[99] Pali, N., "Characterization of Einstein-Fano manifolds via the Kähler-Ricci flow", arXiv: math.DG/0607581.

[100] Paul, S. and G. Tian, "CM stability and the generalized Futaki invariant II", arXiv: math.AG/0605278.

[101] Perelman, G., "The entropy formula for the Ricci flow and its geometric applications", arXiv: math.DG/0211159.

[102] Phong, D.H., J. Ross, and J. Sturm, "Deligne pairings and the Knudsen-Mumford expansion", arXiv: math.DG/0612555, to appear in J. Differential Geom. (2007).

[103] Phong, D.H., N. Sesum, and J. Sturm, "Multiplier ideal sheaves and the Kähler-Ricci flow", Comm. Anal. Geom. 15 (2007), no. 3, 613-632.

[104] Phong, D.H., J. Song, J. Sturm, and B. Weinkove, "The Moser-Trudinger inequality on Kähler-Einstein manifolds", arXiv: math.DG/0604076, to appear in Amer. J. Math. (2007).

[105] Phong, D.H., J. Song, J. Sturm, and B. Weinkove, "The Kähler-Ricci flow and the $\bar{\partial}$ operator on vector fields", arXiv: math 07054048 [math.DG], to appear in J. Differential Geom.

[106] Phong, D.H., J. Song, J. Sturm, and B. Weinkove, "The Kähler-Ricci flow and positive bisectional curvature", arXiv: math 07062852 [math.DG], to appear in Inventiones Math.

[107] Phong, D.H. and J. Sturm, "Stability, energy functionals, and Kähler-Einstein metrics", Commun. Anal. Geom. 11 (2003) 563-597.

[108] Phong, D.H. and J. Sturm, "Scalar curvature, moment maps, and the Deligne pairing", Amer. J. Math. 126 (2004) 693-712.

[109] Phong, D.H. and J. Sturm, "On asymptotics for the Mabuchi energy functional", Abstract and Applied Analysis, 271-286, World Sci. Publ., River Edge, NJ, 2004.

[110] Phong, D.H. and J. Sturm, "The Futaki invariant and the Mabuchi energy of a complete intersection", Commun. Anal. Geom. 12 (2004) no. 1-2, 321-343.

[111] Phong, D.H. and J. Sturm, "On the Kähler-Ricci flow on complex surfaces", Pure Appl. Math. Q. 1 (2005) no. 2, 405-413.

[112] Phong, D.H. and J. Sturm, "On stability and the convergence of the Kähler-Ricci flow", J. Differential Geom. 72 (2006) no. 1, 149-168.

[113] Phong, D.H. and J. Sturm, "The Monge-Ampère operator and geodesics in the space of Kähler potentials", Invent. Math. 166 (2006) 125-149.

[114] Phong, D.H. and J. Sturm, "Test configurations and geodesics in the space of Kähler potentials", J. Symplectic Geom. 5 (2007), no. 2, 221-247.

[115] Phong, D.H. and J. Sturm, "On the $C^{1,1}$ regularity of geodesics defined by test configurations", arXiv: math 07073956 [math.DG].

[116] Popovici, D., "A simple proof of a theorem by Uhlenbeck and Yau", Math. Z. 250 (2005), no. 4, 855-872.

[117] Rollin, Y. and M. Singer, "Non-minimal scalar flat Kähler surfaces and parabolic stability", Invent. Math. 162 (2005) no. 2, 235-270.

[118] Ross, J.A. and R.P. Thomas, "An obstruction to the existence of consta", J. Differential Geom. 72 (2006), no. 3, 429-466. 
[119] Rubinstein, Y., "On the construction of Nadel multiplier ideal sheaves and the limiting behavior of the Kähler-Ricci flow", arXiv: 0708.1950 [math.DG]

[120] Semmes, S., "Complex Monge-Ampere equations and symplectic manifolds", Amer. J. Math. 114 (1992) 495-550.

[121] Sesum, N. and G. Tian, "Bounding scalar curvature and diameter along the Ricci flow (after Perelman) and applications", http://www.math.lsa.umich.edu/ lott/ricciflow/perelman.html.

[122] Simanca, S., "Kähler metrics of constant scalar curvature on bundles over $\mathbf{C P}^{n-1}$ ", Math. Ann. 291 (1991) no. 2, 239-246.

[123] Siu, Y.T., "The existence of Kähler-Einstein metrics on manifolds with positive anticanonical line bundle and a suitable finite symmetry group", Ann. of Math. 127 (1988) 585-627.

[124] Siu, Y.T., "The Fujita conjecture and the extension theorem of Ohsawa-Takegoshi", in Geometric Complex Analysis, Hayama (1995) 577-592, World Scientific.

[125] Siu, Y.T., "Multiplier ideal sheaves in complex and algebraic geometry", Sci. China Ser. A 48 (2005), 1-31.

[126] Siu, Y.T. and S.T. Yau, "Compact Kähler manifolds of positive bisectional curvature", Invent. Math. 59 (1980) 189-204.

[127] Song, J., "The $\alpha$-Invariant on $C P^{2} \# 2 C \bar{P}^{2}$ ", arXiv:math/0205041.

[128] Song, J., "The $\alpha$-Invariant on Toric Fano Manifolds", Amer. J. Math. 127 (2005), no. $6,1247-1259$.

[129] Song, J. and G. Tian, "The Kähler-Ricci flow on surfaces of positive Kodaira dimension", Invent. Math. 170 (2007), no. 3, 609-653.

[130] Song, J. and B. Weinkove, "Energy functionals and canonical metrics", Duke Math. J. 137 (2007) 159-184.

[131] Song, J. and B. Weinkove, "On the convergence and singularities of the J-flow with applications to the Mabuchi energy" Comm. Pure Appl. Math. 61 (2008), no. 2, 210-229.

[132] Song, J. and S. Zelditch, "Convergence of Bergman metrics on CP", arXiv: math / 0703517 [math.DG].

[133] Song, J. and S. Zelditch, "Bergman metrics and geodesics in the space of Kähler metrics on toric varieties", arXiv: 0707.3082 [math.CV].

[134] Song, J. and S. Zelditch, "Test configurations, large deviations and geodesic rays on toric varieties", arXiv:0712.3599.

[135] Struwe, M., "Curvature flows on surfaces", Ann. Sc. Norm. Super. Pisa Cl. Sci. (5) 1 (2002), no. 2, 247-274.

[136] Szekelyhidi, G., "Extremal metrics and K-stability", Bull. Lond. Math. Soc. 39 (2007), no. 1, 76-84.

[137] Szekelyhidi, G., "The Calabi functional on a ruled surface", ArXiv:math/0703562.

[138] Szekelyhidi, G., "Optimal test-configurations for toric varieties", ArXiv:0709.2687.

[139] Tian, G., "On Kähler-Einstein metrics on certain Kähler manifolds with $c_{1}(M)>0$ ", Inventiones Math. 89 (1987) 225-246.

[140] Tian, G., "On a set of polarized Kähler metrics on algebraic manifolds", J. Differential Geometry 32 (1990) 99-130.

[141] Tian, G., "On Calabi's conjecture for complex surfaces with positive first Chern class", Inventiones Math. 101 (1990) 101-172.

[142] Tian, G., "The $K$-energy on hypersurfaces and stability", Comm. Anal. Geometry 2 (1994) 239-265.

[143] Tian, G., "Kähler-Einstein metrics with positive scalar curvature", Inventiones Math. 130 (1997) 1-37.

[144] Tian, G., "Bott-Chern forms and geometric stability", Discrete Contin. Dynam. Systems 6 (2000), no. 1, 211-220. 
[145] Tian, G., "Extremal metrics and geometric stability", Special issue for S. S. Chern. Houston J. Math. 28 (2002), no. 2, 411-432.

[146] Tian, G. and S.T. Yau, "Kähler-Einstein metrics on complex surfaces with $c_{1}(M)$ positive", Comm. Math. Phys. 112 (1987) 175-203.

[147] Tian, G. and Z. Zhang, "On the Kähler-Ricci flow on projective manifolds of general type", Chinese Ann. Math. Ser. B 27 (2006), no. 2, 179-192.

[148] Tian, G. and X.H. Zhu, "Convergence of Kähler-Ricci flow", J. Amer. Math. Soc. 20 (2007), no. 3, 675-699.

[149] Thomas, R., "Notes on GIT and symplectic reduction for bundles and varieties", math.AG/0512411.

[150] Tosatti, V. and B. Weinkove, "The Calabi flow with small initial energy", Math. Res. Lett. 14 (2007), no. 6, 1033-1039.

[151] Tosatti, V., B. Weinkove, and S.T. Yau, "Taming symplectic forms and the CalabiYau equation", arXiv:math/0703773.

[152] Uhlenbeck, K. and S.T. Yau, "On the existence of Hermitian Yang-Mills connections on stable vector bundles", Commun. Pure Appl. Math. 39 (1986) 257-293.

[153] Wang, X., "Balance point and stability of vector bundles over a projective manifold", Math. Res. Lett. 9 (2002), no. 2-3, 393-411.

[154] Wang, X.J. and Z.H. Zhu, "Kähler-Ricci solitons on toric manifolds with positive first Chern class", Advances Math. 188 (2004) 87-103.

[155] Weinkove, B., "A complex Frobenius theorem, multiplier ideal sheaves, and Hermitian-Einstein metrics on vector bundles", Trans. Amer. Math. Soc. 359 (2007), no. 4, 1577-1592.

[156] Weinkove, B., "On the J-flow in higher dimensions and the lower boundedness of the Mabuchi energy", J. Differential Geom. 73 (2006), no. 2, 351-358.

[157] Yau, S.T., "On the Ricci curvature of a compact Kähler manifold and the complex Monge-Ampere equation I", Comm. Pure Appl. Math. 31 (1978) 339-411.

[158] Yau, S.T., "Non-linear analysis in geometry", Enseign. Math. (2) 33 (1987) no. 1-2, 109-158.

[159] Yau, S.T., "Open problems in geometry", Proc. Symposia Pure Math. 54 (1993) $1-28$.

[160] Yau, S.T., "Perspectives of geometric analysis", AMS/IP Studies in Advanced Mathematics 39 (2007) 289-378.

[161] Ye, R., "The logarithmic Sobolev inequality along the Ricci flow", arXiv:0707.2424, [math.DG].

[162] Yotov, M., "On the generalized Futaki invariant", arXiv: math.AG/9907055.

[163] Zelditch, S., "Szegö kernels and a theorem of Tian", Int. Math. Res. Notices 6 (1998) $317-331$

[164] Zelditch, S., "Bernstein polynomials, Bergman kernels, and toric Kähler varieties", arXiv: 0705.2879 [math.CV].

[165] Zhang, Q.S., "A uniform Sobolev inequality under Ricci flow", arXiv: 0706.1594 [math.DG].

[166] Zhang, S.W., "Heights and reductions of semistable varieties", Compositio Math. 104 (1996) $77-105$.

[167] Zhou, B. and X.H. Zhu, "Minimizing weak solutions for Calabi's extremal metrics on toric manifolds", ArXiv:math/0611099.

[168] Zhu, X.H., "Kähler-Ricci flow on toric manifolds with positive first Chern class", arXiv:math 0703486 [math.DG].

Department of Mathematics, Columbia University, New York, NY 10027

Department of Mathematics, Rutgers University, Newark, NJ 07102 